NBER WORKING PAPER SERIES

\title{
INFORMATION SPILLOVERS IN EXPERIENCE GOODS COMPETITION
}

\author{
Zhuoqiong Charlie Chen \\ Christopher T. Stanton \\ Catherine Thomas \\ Working Paper 28584 \\ http://www.nber.org/papers/w28584 \\ NATIONAL BUREAU OF ECONOMIC RESEARCH \\ 1050 Massachusetts Avenue \\ Cambridge, MA 02138 \\ March 2021
}

This work is supported by the National Natural Science Foundation of China (No. 71903046) and the "Shenzhen Peacock Program" start-up research grant. We would like to thank Ricardo Alonso, Yongmin Chen, David de Meza, Hong Feng, Xiangting Hu, Yangguang Huang, Xinyu Hua, Kohei Kawaguchi, Jianpei Li, Jin Li, Albert Ma, Jusso Valimaki, Jie Zheng, and participants at the HKUST Economics Webinar, HKUST IO workshop, and Tsinghua BEAT conference for helpful comments. The views expressed herein are those of the authors and do not necessarily reflect the views of the National Bureau of Economic Research.

NBER working papers are circulated for discussion and comment purposes. They have not been peer-reviewed or been subject to the review by the NBER Board of Directors that accompanies official NBER publications.

(C) 2021 by Zhuoqiong Charlie Chen, Christopher T. Stanton, and Catherine Thomas. All rights reserved. Short sections of text, not to exceed two paragraphs, may be quoted without explicit permission provided that full credit, including $(\odot$ notice, is given to the source. 
Information Spillovers in Experience Goods Competition

Zhuoqiong Charlie Chen, Christopher T. Stanton, and Catherine Thomas

NBER Working Paper No. 28584

March 2021

JEL No. D11,L1,L13,L15,L26,M21

\begin{abstract}
$\underline{\text { ABSTRACT }}$
When experience goods compete, consuming one product can be informative about value for similar untried products. We study a two-period model of duopoly competition in markets that have this feature and where firms can price discriminate between consumers based on purchasing history. Price dynamics, firm profits, and consumer surplus depend on how information spillovers shape demand from the consumers who have trialed the rival product - the potential switchers. In the first period, rather than competing intensely for all future profits, firms compete only for the difference in future profits between repeat and switching consumers. Demand-side information spillovers offer an explanation of how competing firms in new product markets can be profitable in all periods even when selling products that are indistinguishable ex ante.

Zhuoqiong Charlie Chen

B301A, School of Economics and Management

Harbin Institute of Technology, Shenzhen

Shenzhen 518000

China

chenzq926@gmail.com

Christopher T. Stanton

210 Rock Center

Harvard University

Harvard Business School

Boston, MA 02163

and NBER

christopher.t.stanton@gmail.com

Catherine Thomas

Management Department

London School of Economics

Houghton Street

London.

WC2A 2AE

United Kingdom

and CEPR

c.m.thomas@1se.ac.uk
\end{abstract}




\section{Introduction}

In markets where experience goods compete, consumers become informed about their individual values for the goods that they trial. Klemperer (1987b), Klemperer (1995), and Villas-Boas (2006) describe this informational advantage as a switching cost. In this paper, we study markets where an individual consumer's values for competing non-durable experience goods are correlated, so that any trial allows her to learn her value for the trialled good (Nelson (1970)) and also update her perceived value for any rival goods.

We characterize the nature of duopoly competition in a two-period model featuring information spillovers that determine the extent of informational advantage for trialled products. Consumers first choose between two unfamiliar products and an outside alternative of known value. In the second period, consumers face the same choice, but are better-although not fully-informed about their values for the new products. Firms are able to observe past purchases, which permits behaviour-based price discrimination. There are two main findings:

First, even when consumers' have ex-ante identical beliefs about the value of the two products, firms make profits from both repeat and switching consumers in the second period whenever the new products are sufficiently valuable. This ensures firms make positive profits in the overall game whenever there are any switching consumers. Second, the extent of the information spillovers from rival product consumption determines the size of firm profits. Whenever consumer values for the two new products are imperfectly correlated, the trialled product retains some informational advantage. However, for a range of information spillovers, firm profits are decreasing in the informational advantage. Because information spillovers reflect the degree to which consumer values are correlated, this result implies that firm profits can be greater when new rival goods are closer substitutes. The same logic means that consumer surplus can be lower when rival goods are closer substitutes.

The main intuition for these findings is that when product evaluations are more highly correlated, that is, when the products are closer substitutes, consumers become better-informed about their value for each product after initial trial. Consumers therefore sort into subsequent purchases more efficiently, and, for a range of parameter values, switching consumers have a higher expected willingness to pay for the non-trialed product, making them relatively price inelastic. These changes allow price-discriminating firms to extract more profit from the group of switching consumers, which, over some range of information spillovers, outweighs the direct effect of closer product substitutability on second-period profits. In some of the widely-known models where switching costs lock consumers in to subsequent purchase, intense first-period competition for market share means that firms earn zero profits overall. In contrast, here, firms 
compete in the first period only for the differential profits between future repeat and switching consumers rather than for all second-period profits. Hence, when an increase in informational spillovers increases the second-period profitability of switching consumers, overall profits also increase.

Because the model features competing and similar experience goods sold by firms that can price discriminate, we believe it characterizes competition in digital marketplaces where buyers can easily browse varieties of a new product and where other types of switching cost may be relatively low. For example, Klemperer (1987a) describes the high transactions cost of closing an existing checking account and opening another with a competing bank. However, in the growing online banking market in many countries, similar competing apps can be freely and easily downloaded, and recent open banking regulation allows consumers to share data on their past banking activity with multiple providers, reducing explicit switching costs. ${ }^{1}$ On the supply side, new digital marketplaces typically allow sellers to collect more information about consumers' browsing histories and infer their past purchases. Examples of firms that use such consumer information to price discriminate can be found in the travel industry and in home goods retail. ${ }^{2}$ In retail banking in the US, firms often offer tailored bonuses for opening new checking accounts, effectively varying price between repeat and switching or new consumers. ${ }^{3}$

In the model, consumer values for the two new products have a known bivariate normal distribution. The correlation between each consumer's value for the new products is governed by a parameter $\rho$, varying between -1 and +1 , that represents the degree of differentiation between the two new products and is also a measure of information spillovers from product trial. This framework nests Bertrand competition (when $\rho=1$ ) and models of experience good pricing when consumers' values are independent, as in Villas-Boas (2004), when $\rho=0$, and establishes a novel connection between these cases. ${ }^{4}$ For simplicity, there are no other switching costs, network externalities, or fixed costs in the model. ${ }^{5}$

\footnotetext{
${ }^{1}$ For example, details about the UK regulation that ensures consumers can share these data can be found here: https://www.openbanking.org.uk/customers/what-is-open-banking/

${ }^{2}$ Hannak et al. (2014) showed that certain consumers were likely to receive higher prices across a range of online travel sites, although reverse engineering the consumer information the sites used to personalize these prices proved difficult.

${ }^{3}$ For example, Figure A.1, in the Appendix, shows a summary of different promotional offers available to new Citizens Bank customers, downloaded from https://www.creditdonkey.com/promotions/citizens-bank/ on $2 / 24 / 2021$. While existing customers of the bank are not offered a bonus, customers likely to be switching from a competitor can earn $\$ 300$ or $\$ 400$ to open a new checking account online, and parents opening an account for a child under 12 are offered $\$ 1000$ when the child turns 18 .

${ }^{4}$ Villas-Boas (2004) assumes that products are horizontally and vertically differentiated and consumers learn about the quality of products from experience.

${ }^{5}$ While these additional features are often present in the digital marketplaces we have in mind, the mechanisms and results laid out in this paper do not rely on their presence, and are mostly robust to extensions that include them. In our setting, switching arises because consumption changes the information structure for consumers with heterogeneous values for the new products.
} 
The value of the new product market is defined as the extent to which the expected value for either of the new products less marginal cost exceeds the value of the outside alternative, and is assumed to be exogenous. Firms compete in prices and the equilibrium outcomes of interest are the intensity of first-period price competition and overall firm profits, as well as consumer surplus in the new market. The analysis shows that these outcomes are all determined by the extent of switching to the rival product after initial trial and the price elasticity of any switching customers relative to the price elasticity of repeat customers. Switching behavior depends on the interaction between the value of the new product market and the magnitude of information spillovers from experience.

Although the equilibrium price charged to repeat customers is always at least as large as the price charged to switchers, information spillovers can increase the share of switchers even to the extent that the mass of switching consumers is more profitable than the mass of repeat consumers. In this case, firms have no incentive to compete intensively in the first period for consumers who will become repeat buyers, and therefore set a first-period price that exceeds marginal cost. In this case, firms not only earn positive profits overall, but also earn positive profits in each period.

In the large literature on experience good pricing building on Nelson (1970), the majority of papers do not model competition between firms. Cremer (1984) studies a two-period setup allowing price discrimination by a monopolist, and shows that the first-period price can exceed the second-period price because consumers' willingness to pay in the first period includes the option value of learning from consumption. Bergemann and Välimäki (2006) consider partial learning from consuming an experience good in a continuous time model and show that price dynamics are determined by whether the market is mass or niche, a distinction that also plays a role in this paper. ${ }^{6}$ Jing (2011) extends their study to evaluate the welfare implications of price discrimination and price commitment. Firm profit and social welfare are greater under price commitment in a niche market and greater under price discrimination in a mass market.

When competing products are considered and price discrimination is not possible, there arises a well-known trade off in later periods between setting a uniform price that extracts surplus from repeat loyal customers while also attracting customers of rival brands to switch. Villas-Boas (2004) studies this trade off in a two-period Hotelling model with no learning about rival products from consumption. In that model, second-period prices are related to first-period market share. Villas-Boas (2006) studies experience good pricing in an infinite horizon model

\footnotetext{
${ }^{6}$ Their definition of mass market coincides with the equilibrium that exists in this paper where the expected value of the new product exceeds the marginal cost. We show that when this condition is not met, various equilibria can exist, one of which coincides with their niche market definition.
} 
with overlapping generations of consumers and shows how firms may compete intensively in prices to gain future market share even if consumers anticipate that lower prices now means higher prices later. Doganoglu (2010) shows that when firms are restricted to use uniform prices, prices are lower when switching costs are sufficiently low than when they are absent. In our model, price discrimination allows firms to avoid the trade off that arises with a uniform price of reducing profits from repeat consumers in order to attract switching consumers.

Our setting involves consumers having unknown and heterogeneous values for two products competing with a known alternative. Bergemann and Välimäki (1997) study the related case of a new entrant competing with a known incumbent. Their model features a different type of information spillover, in that public beliefs about the common-value component of the new good are updated after any consumer experience. ${ }^{7}$ Both firms want to speed up information transmission, and, since only trials of the new product are informative, the incumbent's incentive to compete with the entrant on price is softened. Consumer preferences are also horizontally differentiated, with consumers being distributed uniformly on a line, and both firms prefer maximum ex post differentiation in this setting. In our model, within-consumer information spillovers inform each consumer's private values. Because these spillovers vary with the extent of product differentiation, competing firms can prefer to be closer substitutes.

In the broader literature on switching costs (Klemperer, 1987a,b; Farrell and Shapiro, 1988; Beggs and Klemperer, 1992; Padilla, 1992, 1995; Klemperer, 1995; Farrell and Klemperer, 2007), these costs are usually assumed to be high enough that no consumer switches in equilibrium, and consumer values are assumed to be high enough that no consumer leaves the market after trying one of the goods. Alternatively, in the model by Chen (1997), consumers do switch when they have private switching costs and firms can make monetary transfers to switching customers. Paying customers to switch serves as a form of behavior-based price discrimination, which, as in our model, neutralizes any influence of first-period market-share on the second-period prices. ${ }^{8}$

Fudenberg and Tirole (2000) consider customer poaching in a horizontally differentiated market where consumers know their values and switching incurs no cost. Brand switching only happens when there is behavior-based price discrimination. Consumers switch brands for lower prices and hence inefficient switching arises in equilibrium as some consumers switch to a less-preferred brand. In contrast, in our paper, switching is always welfare improving. This is because unsatisfied consumers have the opportunity to switch and obtain positive surplus rather than leave the new market.

\footnotetext{
${ }^{7}$ Moretti (2011) also models market-level social learning about product quality through reviews.

${ }^{8}$ Chen and Pearcy (2010) show that loyal customers may be rewarded by a lower price if firms can commit to future prices. In this paper, we assume that the distribution of consumer values is ex ante equivalent across brands and firms cannot commit to future prices.
} 
Our interest lies in characterizing how a demand-side information externality increases the profits of competing new products when consumer valuations are unknown prior to trial. We emphasize how information shapes whether consumers remain loyal, switch to a rival product, or leave the new market. Hence, allowing consumers to opt out of the new market altogether is an important feature of the model. The strength of information spillovers from an initial consumption experience, together with the value of the new market, determine the extent of market coverage in the second period, that is, the share of consumers who buy either of the new products rather than exit and purchase the old technology.

Firms can make higher profits when new products are closer substitutes because information spillovers are increasing in the extent of perceived product similarity. Other papers have set out alternative reasons why firms may prefer partial differentiation. For example, Bester (1998) combines price as a signal of quality with the theory of spatial competition and finds that imperfect information about goods' vertical quality characteristics reduces firms' incentives to differentiate horizontally. Gehrig and Stenbacka (2004) study endogenous horizontal product differentiation between goods without the experience good feature and allow switching costs to be increasing in the extent of differentiation.

The demand-side information spillovers that lead to new product profitability in this model differ from both the technology and product market rivalry spillovers created when firms undertake R\&D as described in Bloom et al. (2013). Their market rivalry spillovers refer to the business-stealing effect on firm performance of an innovation in a closely competing product. In our case, with incomplete information about consumer values, a rival's new product generates positive demand for other similar products.

While early competition between close rivals is often modeled as a war of attrition where one dominant firm eventually prevails, equilibria in our model feature two firms that can be profitable in both periods. We show that information spillovers allow firms to remain profitable even when the expected value of the new market is negative, that is, when the expected value of each new product less its marginal cost is outweighed by the known value of the old technology. This is because uninformed consumers purchase a new product for the option value of remaining in the new market once informed, and price discriminating firms facilitate efficient sorting by better-informed consumers into repeat purchase, switching to the rival good, and returning to the old technology.

The paper proceeds as follows. Section 2 introduces the primitives of the model. Section 3 presents the equilibria of the second-stage sub-game without information spillovers $(\rho=0)$ and then the second-stage sub-game with information spillovers $(\rho \in(0,1))$. Section 4 presents the 
full equilibria and analyzes the range of market values for which firms make profits and the range of market values for which profits are zero, both in the case when $\rho=0$ and then when $\rho \in(0,1)$. The proofs that are not in the main text are provided in Appendix B. Section 5 describes some implications of the earlier analysis and presents a theorem relating firm profits to the ability to attract switching consumers. It also provides a numerical example of profits, consumer surplus, and social surplus as a function of market value at different levels of information spillovers. The equilibria when consumers' preferences are negatively correlated $(\rho<0)$ are presented in Appendix C. Appendix D gives the price elasticities of interest. Appendix E presents some discussion of a benchmark case where consumers are fully informed of their values for the new products. Consumer valuations are asymmetric in Appendix F, and firms are unable to price discriminate based on purchasing behavior in Appendix G. Section 6 concludes.

\section{Primitives of the Model}

There are two firms that offer non-durable experience goods A and B, respectively. The marginal cost for both firms is $c \geq 0$. The firms compete by setting prices simultaneously in each of two periods. There exists a unit mass of consumers, each of whom buys one unit of good A or good B, or returns to the outside option, in each period. Firms and consumers have a common discount factor $\delta \in[0,1]$.

Valuations: Each consumer's per period valuation for good $i \in\{A, B\}$ is denoted $\theta_{i}$, and is normalized relative to the existing alternative option value of zero. For simplicity, $\theta_{i}$ is constant across the two periods, but more complicated models could allow consumers to update beliefs about a dynamic process. In the first period, consumers do not know their valuations but know that the joint distribution is bivariate normal $\mathcal{N}(\boldsymbol{\mu}, \boldsymbol{\Sigma})$, where

$$
\boldsymbol{\mu}=\left(\begin{array}{c}
\mu_{A} \\
\mu_{B}
\end{array}\right), \boldsymbol{\Sigma}=\left(\begin{array}{cc}
\sigma_{A}^{2} & \rho \sigma_{A} \sigma_{B} \\
\rho \sigma_{B} \sigma_{A} & \sigma_{B}^{2}
\end{array}\right)
$$

We assume that $\mu_{A}=\mu_{B}=\mu$ and $\sigma_{A}=\sigma_{B}=\sigma>0$, and that $\rho \in[0,1] \cdot{ }^{9} F(\cdot)$ denotes each product's marginal value distribution, and, hence, $F(\cdot)$ is the c.d.f of a univariate normal distribution with mean $\mu$ and variance $\sigma$. The corresponding p.d.f. is denoted $f(\cdot)^{10}$ Note

\footnotetext{
${ }^{9}$ We analyze the $\rho \in[-1,0)$ case in the Appendix B.

10 The probability density function of a bivariate normal distribution is given by

$$
f(x, y)=\frac{1}{2 \pi \sigma_{X} \sigma_{Y} \sqrt{1-\rho^{2}}} \exp \left(-\frac{1}{2\left(1-\rho^{2}\right)}\left[\frac{\left(x-\mu_{X}\right)^{2}}{\sigma_{X}^{2}}+\frac{\left(y-\mu_{Y}\right)^{2}}{\sigma_{Y}^{2}}-\frac{2 \rho\left(x-\mu_{X}\right)\left(y-\mu_{Y}\right)}{\sigma_{X} \sigma_{Y}}\right]\right),
$$
}

and the marginal distribution for one variable is obtained by integrating over the other variable, e.g., the marginal 
that $\theta_{i}$ satisfies the monotone reverse hazard rate (MHR) condition, so that $f(\cdot) /[1-F(\cdot)]$ is an increasing function, whereas $f(\cdot) / F(\cdot)$ is a decreasing function. The joint distribution is common knowledge to both firms and consumers.

Learning: After consuming in the first period, a consumer who bought good $i$ learns her $\theta_{i}$, as in Nelson (1970), and also updates her expectation about $\theta_{-i}$, which is $E\left(\theta_{-i} \mid \theta_{i}\right)$ :

$$
E\left(\theta_{-i} \mid \theta_{i}\right)=\mu_{-i}+\rho \frac{\sigma_{-i}}{\sigma_{i}}\left(\theta_{i}-\mu_{i}\right)=\rho \theta_{i}+(1-\rho) \mu
$$

The parameter $\rho$ therefore measures the information spillover from consumption between the tried and untried new products.

Prices: Firms announce their prices at the beginning of each period simultaneously. The firstperiod price of firm $i$ is denoted $p_{i}$, the second-period price charged to repeat consumers is $R_{i}$, and the second-period price charged to switching consumers is $S_{i}$. Throughout the paper, we assume that firms can price discriminate between consumers based on their purchasing history and so $R_{i}$ may differ from $S_{i}$. Firms cannot distinguish between switching consumers and those who did not purchase in the first period, so both are offered the same price. We do not allow firms to offer two-period subscriptions in the first period, nor can they commit to future prices. ${ }^{11}$

Consumer payoffs: In each period, each consumer can buy only one unit of one good or she can choose to take the outside option that she values at zero. ${ }^{12}$ A consumer who buys good $i$ in the first period obtains a second period payoff of $u_{2}\left(\theta_{i}\right)=\max \left\{\theta_{i}-R_{i}, \rho \theta_{i}+(1-\rho) \mu-S_{-i}, 0\right\}$ if she chooses to continue buying good $i$, switch to good $-i$, or take the outside option in the second period, respectively.

Cutoff consumer types: We define the following cutoff types for firm $i$ 's $(i \in\{A, B\})$ firstperiod consumer who is indifferent between her period-two options.

- $\theta_{i}^{R S}$ : the marginal repeat consumer who is indifferent between buying good $i$ again and distribution of $X$ is given by

$$
f_{X}(x)=\int_{-\infty}^{+\infty} f(x, y) d y=\frac{1}{\sqrt{2 \pi} \sigma_{X}} \exp \left(-\frac{\left(x-\mu_{X}\right)^{2}}{2 \sigma_{X}^{2}}\right) .
$$

11 Suppose both firms offer a two-period subscription at a lump sum price and the cost of return/refund is prohibitively high, then they are effectively competing in a single-period Bertrand competition because goods are ex ante homogeneous to consumers. Suppose instead that both firms can commit to second-period prices and price discrimination is not allowed, then they charge the marginal cost in both periods and earn a zero overall profit. The relevant analysis for price commitment is available upon request.

${ }^{12}$ We interpret the outside option as an "old", tried-and-tested product that remains available to the consumer in each period. 
switching to good $-i$, i.e., $\theta_{i}^{R S}-R_{i}=E\left(\theta_{-i} \mid \theta_{i}^{R S}\right)-S_{-i}$. Hence, with $\rho \neq 1$ we have

$$
\theta_{i}^{R S}=\mu+\frac{R_{i}-S_{-i}}{1-\rho}
$$

- $\theta_{i}^{R O}$ : the consumer who is indifferent between buying good $i$ again and the outside option,

$$
\theta_{i}^{R O}=R_{i}
$$

- $\theta_{i}^{S O}$ : the consumer who is indifferent between switching to good $-i$ and the outside option, i.e., $\rho \theta_{i}^{S O}+(1-\rho) \mu=S_{-i}$. Hence, we have

$$
\theta_{i}^{S O}=\frac{S_{-i}-(1-\rho) \mu}{\rho}
$$

Note that $\theta_{i}^{R S}=\mu$ if $R_{i}=S_{-i}$, suggesting exactly half of firm $i$ 's consumers would switch if firm $i$ 's price to repeat consumers were equal to firm $-i$ 's price to switching consumers. Also, when $\rho>0$, we have $\lim _{\rho \rightarrow 0^{+}} \theta_{i}^{S O}=+\infty$ if $S_{-i}>\mu$ and $\lim _{\rho \rightarrow 0^{+}} \theta_{i}^{S O}=-\infty$ if $S_{-i}<\mu$.

Monopoly: In some equilibria, firms have monopoly power in a share of the market. We define price $M$ as the monopoly price given the demand curve $1-F(\cdot)$. That is, $M$ satisfies

$$
M=\frac{1-F(M)}{f(M)}+c,
$$

and according to the MHR condition, $M$ is uniquely determined.

Denote by $\pi(M)=\int_{M}^{\infty}(M-c) d F(x)$ the profit made by a monopoly firm from charging price $M$ in a market with demand $1-F(M) . S S(M)=\int_{M}^{\infty}(x-c) d F(x)$ is the corresponding social surplus. In the duopoly environment we consider, the demand curve actually faced by each of the firms depends on the nature of equilibrium. ${ }^{13}$

\section{Major innovation and minor innovation:}

The value of the new products relative to the old technology is exogenously given. For convenience, we divide new products into two groups:

- A product is defined as a major innovation if and only if at least half of the consumers value it at above the marginal cost, so $\mu \geq c$.

\footnotetext{
${ }^{13}$ In the absence of a competitor, a monopolist selling two products would extract more total surplus from all consumers' willingness to pay in both periods. This is because the monopolist does not face the duopolists' challenge of competing in first-period price for the most profitable group of consumers in the second period. The role that the competition channel plays in the main results is discussed later.
} 
- A product is a minor innovation if and only if less than half of the consumers value it at above the marginal cost, so $\mu<c$.

In other words, a new market with a minor innovation is preferred to the old technology only by those consumers with high valuations, but a new market with a major innovation is preferred to the old technology for most consumers.

Benchmark case where $\rho=1$

If $\rho=1$, consumers are fully informed about their valuations for both products after the first period and the valuations are equal for both products. This becomes standard Bertrand competition in each period. Because each firm has an incentive to reduce price in the second period, the second-period equilibrium price is equal to the marginal cost, and firms make zero profit. In the first period, since a repeat and a switching customer in the second period both generate profits of zero, there is no incentive to reduce first-period price to fight for future customers. Firms, hence, set first-period price equal to the marginal cost in competing for first-period profit. The consumer surplus in such a setting is equal to the social surplus:

$$
C S_{\rho=1}=(\mu-c)+\delta \int_{c}^{+\infty}(x-c) d F(x)
$$

\section{Second-period equilibria when $\rho \geq 0$}

To characterize the sub-game perfect equilibria of the two-stage pricing game, we start by characterizing possible equilibria in the second-period sub-game. We focus on pure-strategy symmetric sub-game perfect equilibria throughout, where firms choose their prices simultaneously in each period. Firms' second-period profit consists of two parts that can be written in general form as:

$$
\underbrace{\left(R_{i}-c\right) \cdot \lambda_{i} \int_{\max \left(\theta_{i}^{R S}, \theta_{i}^{R O}\right)}^{+\infty} d F\left(\theta_{i}\right)}_{\text {profit from repeat customers }}+\underbrace{\max \left(0,\left(S_{i}-c\right) \cdot \lambda_{-i} \int_{\theta_{-i}^{S O}}^{\theta_{-i}^{R S}} d F\left(\theta_{-i}\right)\right)}_{\text {profit from switching customers }},
$$

where $\lambda_{i}$ denotes firm $i$ 's market share in the first period and $\lambda_{i}+\lambda_{-i}=1$. Because we allow behavior-based price discrimination such that the price to repeat customers $R_{i}$ can differ from the price to switching customers $S_{i}$, we will see that the optimal prices are independent of the first period market share $\lambda_{i}{ }^{14}$ For the $\rho>0$ case, we will show in Proposition 2 that

\footnotetext{
${ }^{14}$ In an alternative setting with uniform pricing, as is generally the case in the literature on dynamic pricing for experience goods (Doganoglu, 2010; Villas-Boas, 2006), first-period market share does affect second-period prices because the firm with larger first-period market share would charge a higher second-period price. This
} 
there are three types of second-period equilibria, illustrated in Figures 1, 2, and 3. In each of the figures, the $x$-axis is the consumer valuation for the trialed product, $\theta_{i}$, and the $y$-axis is consumer surplus. In each, the line with the steeper slope represents the consumer's surplus from purchasing product $i$ again in the second period, $\left(\theta_{i}-R_{i}\right)$. The consumer's surplus from switching to the untried product, $E\left(\theta_{-i} \mid \theta_{i}\right)-S_{-i}=\rho \theta_{i}+(1-\rho) \mu-S_{-i}$ is the flatter-but still positively-sloped-line in each figure. ${ }^{15}$

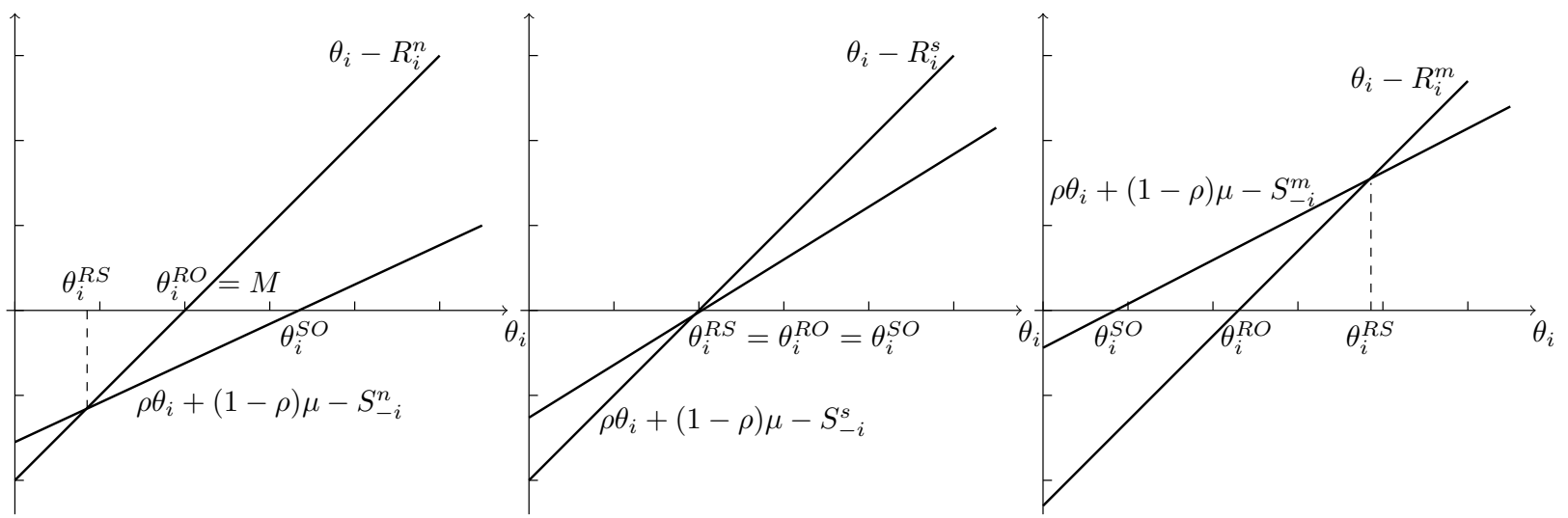

Figure 1: Niche market equilibrium, where $R_{i}^{n}=M$ and $S_{-i}^{n} \geq c$.
Figure 2: Semi-niche market equilibrium, where $R_{i}^{s} \in[m, M]$ and $S_{-i}^{s}=\rho R_{i}^{s}+(1-\rho) \mu$
Figure 3: Mass market equilibrium, where $R_{i}^{m}, S_{-i}^{m}$ are given by (8), (9).

First, when $\mu$ is sufficiently small, there exists a "niche market" equilibrium (see Figure 1 for an example with $\rho>0$ ) in which the marginal repeat consumer strictly prefers the outside option and the new market is not fully covered. The consumers that have a bad initial experience choose to leave the market rather than to switch to the untried product, so $\max \left(\theta_{i}^{R S}, \theta_{i}^{R O}\right)=\theta_{i}^{R O}$, there are no switchers, and the second maximum in (5) takes on the value of zero because $\theta_{-i}^{S O}>\theta_{-i}^{R S}{ }^{16}$ Since no consumers switch in equilibrium, each firm makes monopoly profit in the second period in its respective share of the market by charging the monopoly price $M$. In order for such a price to repeat consumers be part of an equilibrium, it must be true that $\mu$ is sufficiently small-i.e. consumers expect to have sufficiently low valuations for the untried product - to guarantee that

firm's repeat customers are informed and hence are less price elastic, whereas the opposing firm's customers are less informed and more price elastic. Therefore, the "average" price elasticity of the consumers is lower when a greater share customers are informed and the firm therefore charges a higher price. We instead assume that the firms can identify repeat consumers from switching consumers according to past purchasing history. Hence, each firm chooses optimal prices for repeat and switching consumers separately according to each group's price elasticity, and the initial market share has no effect on these prices.

${ }^{15}$ The positive slope of switchers' demand contrasts with the $\rho=0$ case, where the consumer's surplus from switching is a constant, independent of $\theta_{i}$. When $\rho \in(0,1)$, consumer's surplus from switching is positively correlated with $\theta_{i}$.

${ }^{16}$ In particular, when $\rho>0$, we have $\theta_{-i}^{S O}>\theta_{-i}^{R S}$ in the niche market equilibrium as is shown in Figure 1. As $\rho$ approaches zero, $\theta_{-i}^{S O}$ approaches $+\infty$ when $S_{-i}>\mu$, which always holds in the niche market equilibrium according to Proposition 1. Hence, $\theta_{-i}^{S O}>\theta_{-i}^{R S}$ also holds for $\rho=0$. In both cases, the second term in (5) equals zero. 
no firm can charge a price $S_{-i} \geq c$ to attract switching consumers.

Second, when $\mu$ is sufficiently large, there exists a "mass market" equilibrium (see Figure 3 for an example of the $\rho>0$ case) in which the marginal repeat consumer strictly prefers to stay in the market rather than to leave the market and take the outside option. That is, at least some of the consumers who have a bad initial experience switch to the untried product for which they have a high conditional expectation, the second maximum in (5) takes on the value of the non-zero term, as $\theta_{-i}^{R S}>\theta_{-i}^{S O} \geq-\infty$, and the firm makes some profits from switchers. ${ }^{17}$ Whether all or some customers switch to the untried product is determined by $\rho$. When a mass market equilibrium exists, we can derive the second-period equilibrium prices via the first order approach.

Third, when $\mu$ takes on intermediate values, there exists an equilibrium that is neither the mass nor the niche market equilibrium where the marginal repeat consumer is indifferent between consuming again in the market and the outside option. We refer to this equilibrium as the "semi-niche market equilibrium" (see Figure 2 for an example with $\rho>0$ ). In this equilibrium, no consumer switches brand after a bad initial experience - similar to the niche market equilibrium - but a marginal deviation of charging slightly lower prices to switching consumers results in some switching.

\subsection{No Information Spillovers, i.e. $\rho=0$}

When $\rho=0$, there is no correlation between customers' valuations so customers who consumed good $i$ in the first period can make no inference about good $-i$ in the second period. Proposition 1 provides the sufficient and necessary conditions for each of the three equilibria in this case.

Proposition 1. Suppose $\rho=0$ and all consumers have purchased one of the goods. Then, in the second period there exists a unique equilibrium, which is:

- a niche market equilibrium where $R_{i}^{n}=M$ and $S_{i}^{n} \geq c$ if and only if $\mu \leq c$;

- a semi-niche market equilibrium where $R_{i}^{s}=M$ and $S_{i}^{s}=\mu$ if and only if $c \leq \mu \leq M ; ;^{18}$

- a mass market equilibrium where $R_{i}^{m}=S_{i}^{m}=\frac{1}{2 f(\mu)}+c<M$ if and only if $\mu>M$.

Proposition 1 states that the sufficient and necessary condition for a mass market equilibrium to exist is $\mu>M$, which is consistent with the standard definition of "mass market" in the literature (Bergemann and Välimäki, 2006; Ivanov, 2009). We thus follow the literature in

\footnotetext{
${ }^{17}$ Recall that when $\rho>0, \lim _{\rho \rightarrow 0^{+}} \theta_{-i}^{S O}=-\infty$ if $S_{i}<\mu$, which holds in the mass market equilibrium according to Proposition 1.

${ }^{18}$ Note that when $\mu=c$ the semi-niche and the niche market equilibrium coincide.
} 
naming the equilibrium as such. Note that the new product market is fully covered in this mass market equilibrium - all consumers buy $i$ or $-i$ in the second period.

Prior studies define a "niche market" as existing if and only if $\mu<M$. Yet Proposition 1 shows that there are two different equilibria for this parameter range. We refer to the equilibrium with no switchers as the niche market because it has the feature that only those consumers who have a high valuation for the products remain in the new market.

Firms' market power among repeat consumers is greater in the semi-niche than in the mass market equilibrium, and greater still in the niche market equilibrium, because $R_{i}^{m}<R_{i}^{s}=R_{i}^{n}$. Firms' market power among switching consumers has the opposite ordering: it is greatest in the mass market equilibrium, then the semi-niche and then absent in the niche market equilibrium. This is because $S_{i}^{m}>S_{i}^{s}$ and the niche market equilibrium has no switchers.

We provide a sketch of the proof for the mass market equilibrium. According to the earlier discussion, the mass market equilibrium with $\rho=0$, given in Proposition 1 , is characterized by restricting $\theta_{i}^{R S}>\theta_{i}^{R O}$ and $\theta_{-i}^{S O}=-\infty$ in the second-period profit, equation (5). ${ }^{19}$ First order conditions of equation (5) w.r.t $R_{i}$ and $S_{i}$, respectively, yield

$$
R_{i}^{m}=\frac{1-F\left(\theta_{i}^{R S}\right)}{f\left(\theta_{i}^{R S}\right)}+c \quad \text { and } \quad S_{i}^{m}=\frac{F\left(\theta_{-i}^{R S}\right)}{f\left(\theta_{-i}^{R S}\right)}+c .
$$

By the definition of $\theta_{i}^{R S}$ given in equation (1), we have

$$
\theta_{i}^{R S}=\mu+\frac{1-2 F\left(\theta_{i}^{R S}\right)}{f\left(\theta_{i}^{R S}\right)}
$$

In fact, the only $\theta_{i}^{R S}$ that satisfies (7) is $\theta_{i}^{R S}=\mu$, as the LHS of (7) increases in $\theta_{i}^{R S}$ and the RHS of (7) decreases in $\theta_{i}^{R S}$. Replacing $\theta_{i}^{R S}$ and $\theta_{-i}^{R S}$ with $\mu$ in (6) gives $R_{i}^{m}=S_{i}^{m}=\frac{1}{2 f(\mu)}+c .^{20}$

Appendix D derives the own- and cross-price elasticities for each of the three equilibria. A comparison of the own-price elasticities in the mass and niche market equilibria proves relevant to the future discussion. In the mass market case, the own-price elasticities for repeat and switching customers, respectively, are $E_{R_{i}^{m}}^{D_{i}^{R}}=-\frac{R_{i}^{m}}{R_{i}^{m}-c}$ and $E_{S_{i}^{m}}^{D_{i}^{S}}=-\frac{S_{i}^{m}}{S_{i}^{m}-c}$. Because $R_{i}^{m}=S_{i}^{m}$, the firm's market power in each segment - summarized by the Lerner index- is $\frac{R_{i}^{m}-c}{c}$. In the niche market equilibria, the own-price elasticity for repeat customers is $E_{R_{i}^{n}}^{D_{i}^{R}}=-\frac{M}{M-c}$, and there are no switching customers in equilibrium. The Lerner index for repeat consumers is, hence, $\frac{M-c}{c}$. Because $R_{i}^{m}<M$, the market power of each firm in the niche market equilibrium

\footnotetext{
${ }^{19}$ The semi-niche market is characterized by construction.

${ }^{20}$ Observing in the mass market equilibrium that the willingness to pay of all switching customers is equal to $\mu$, which is greater than $M$, shows that it is the competition between the two firms that leads to optimal prices to repeat and switching customers being equal to each other at a level below $M$.
} 
exceeds the market power in the mass market equilibrium.

Figure 4 illustrates the niche market equilibrium, Figure 5 illustrates the semi-niche market equilibrium, and Figure 6 the mass market equilibrium for the $\rho=0$ case. The horizontal line in each figure is the expected value of switching to $-i$ in the second period, $\left(\mu-S_{-i}^{k}\right)$, where $k=n, s, m$ in the niche, semi-niche, and mass market equilibria, respectively.

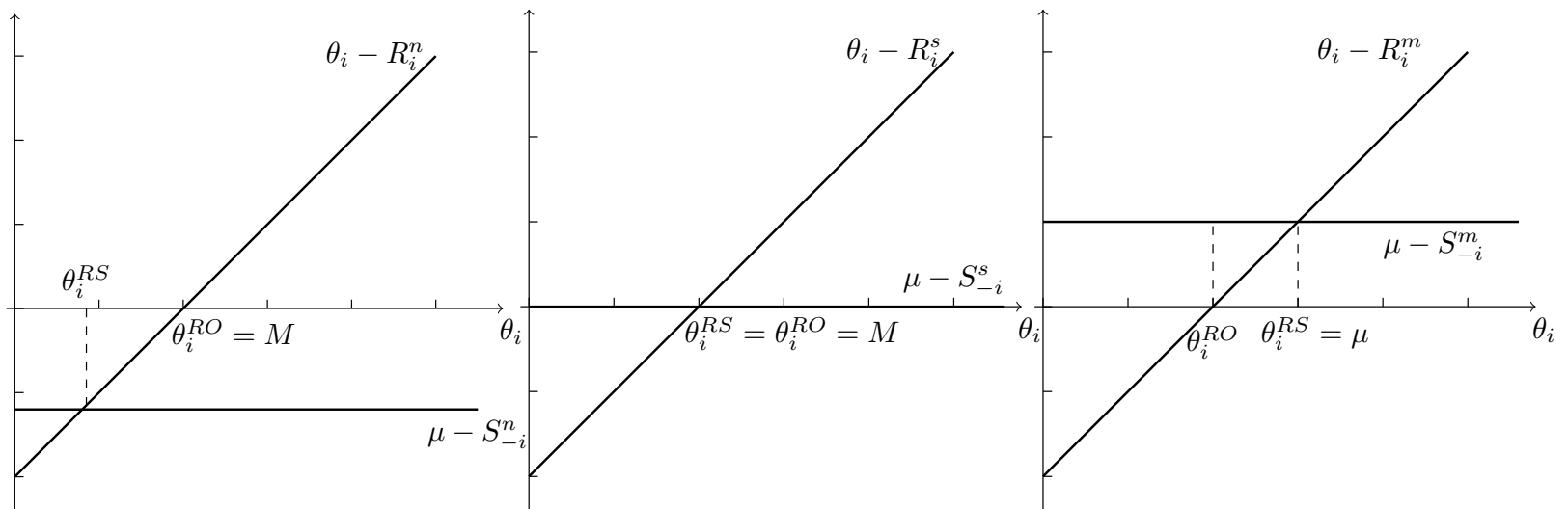

Figure 4: Niche market equilibrium, where $R_{i}^{n}=M$.
Figure 5: Semi-niche market equilibrium, where $R_{i}^{s}=M, S_{-i}^{s}=\mu$.
Figure 6: Mass market equilibrium, where $R_{i}^{m}=S_{-i}^{m}=1 /[2 f(\mu)]+c$.

Corollary 1 describes firm profits in each second-period equilibrium.

Corollary 1. Suppose $\rho=0$ and all consumers have purchased one of the goods. In the second period,

- firm $i$ 's profit in the niche market equilibrium consists of $\lambda_{i} \pi(M)$ from repeat consumers and zero profit from switching consumers (of which there are none).

- firm i's profit in the semi-niche market equilibrium consists of $\lambda_{i} \pi(M)$ from repeat consumers and $\lambda_{-i}(\mu-c) F(M)$ from switching consumers;

- firm i's profit in the mass market equilibrium consists of $\frac{\lambda_{i}}{4 f(\mu)}$ from repeat customers and $\frac{\lambda_{-i}}{4 f(\mu)}$ from switching consumers.

In fact, the $\rho=0$ setting is a special case of $\rho \leq 0$, which we analyze in Appendix C. All equilibrium choices in the former can be obtained by setting $\rho=0$ in the equilibrium choices in the latter.

\subsection{With Information Spillovers, i.e. $\rho \in(0,1)$}

In the presence of information spillovers, $\rho \in(0,1)$, the three equilibria are those shown earlier in Figures 1 - 3. In none of these three equilibria is the new market fully covered. Because 
consumer values for the products are positively correlated, a share of consumers always returns to the outside option after trying one of the new products. ${ }^{21}$

The earlier discussion showed that in the niche market equilibrium (Figure 1) we have $\max \left(\theta_{i}^{R S}, \theta_{i}^{R O}\right)=\theta_{i}^{R O}$ and the profit from switchers in (5) equals zero. The optimal secondperiod price to repeat customers is exactly the monopoly price, $M$, as it was when $\rho=0$. The existence of such an equilibrium, then, requires that the competitor cannot poach customers with a price at least as large as marginal $\operatorname{cost} c$. The sufficient and necessary condition for the equilibrium, as given below by Proposition 2, guarantees that the competing firm cannot do so.

In the mass market equilibrium (Figure 3), $\max \left(\theta_{i}^{R S}, \theta_{i}^{R O}\right)=\theta_{i}^{R S}$ and the profit from switchers expression in (5) takes the value of the non-zero term because $\theta_{-i}^{R S}>\theta_{-i}^{S O}$. When a mass market equilibrium exists, we can derive the second-period equilibrium prices by the first order approach:

$$
\begin{aligned}
R_{i}^{m} & =\frac{(1-\rho)\left[1-F\left(\theta_{i}^{R S}\right)\right]}{f\left(\theta_{i}^{R S}\right)}+c, \\
S_{i}^{m} & =\frac{(1-\rho)\left[F\left(\theta_{-i}^{R S}\right)-F\left(\theta_{-i}^{S O}\right)\right]}{f\left(\theta_{-i}^{R S}\right)+\frac{1-\rho}{\rho} f\left(\theta_{-i}^{S O}\right)}+c .
\end{aligned}
$$

By switching between $i$ and $-i$ in (9), we can solve for the unique $R_{i}^{m}$ and $S_{-i}^{m} \cdot{ }^{22}$ We show the uniqueness of $R_{i}^{m}$ and $S_{i}^{m}$ in the proof of Proposition 2, but the intuition is straightforward, as the right hand sides of (8) and (9) are decreasing in $R_{i}^{m}$ and $S_{i}^{m}$, respectively.

In the semi-niche market equilibrium (Figure 2), the probability of consumer switching is zero. The marginal repeat consumer is indifferent between consuming in the new market and taking the outside option and consumers with lower valuations strictly prefer the outside option. We can obtain the price to repeat consumers in a semi-niche market equilibrium by letting $R_{i}^{m}=\theta_{i}^{R S}=m$ in (8). Hence, $m$ satisfies

$$
m=\frac{(1-\rho)[1-F(m)]}{f(m)}+c
$$

The price $m$ ensures the marginal repeat consumer with $\theta_{i}^{R S}$ is indifferent between repeat purchase and the outside option, due to $\theta_{i}^{R S}-m=0 .{ }^{23}$ On the other hand, we can construct the price $\rho m+(1-\rho) \mu$ which ensures that the marginal repeat customer $\theta_{i}^{R S}$ is indifferent

\footnotetext{
${ }^{21}$ In Appendix G, we provide analysis of second-period equilibrium under uniform pricing, i.e., when firms cannot price discriminate, given that $\rho \geq 0$. We show that there only exist a mass and a niche market equilibrium.

${ }^{22}$ Note that $R_{i}^{m} \rightarrow c$ and $S_{i}^{m} \rightarrow c$ when $\rho \rightarrow 1$. This is consistent with the case where $\rho=1$, discussed at the end of Section 2.

${ }^{23}$ According to the MHR condition, $m$ is uniquely determined. Also, $m=M$ when $\rho=0$ and $m<M$ when $\rho>0$.
} 
between switching and the outside option. ${ }^{24}$ There is, in fact, a continuum of semi-niche market equilibria, and the equilibrium we just characterized is the one with the lowest price.

The value of the new product market, $\mu$, determines which equilibrium applies. The relevant ranges for each equilibrium when $\rho \in(0,1)$ differ from the relevant ranges for when $\rho=0$. All equilibria are shown in Proposition 2.

Proposition 2. Suppose $\rho \in(0,1)$ and all consumers have purchased one of the goods. In the second period there exists:

- a unique niche market equilibrium where firm $i$ charges $R_{i}^{n}=M$ (and any $S_{i}^{n} \geq c$ ) if and only if $\mu \in\left(-\infty, \frac{c-\rho \cdot M}{1-\rho}\right)$;

- a continuum of semi-niche market equilibria where firm $i$ charges $\forall R_{i}^{s} \in[m, \iota]$ and $S_{i}^{s}=$ $\rho R_{i}^{s}+(1-\rho) \mu$ if and only if $\mu \in\left(-\infty, \frac{c-\rho \cdot \iota}{1-\rho}\right]$, given any $\iota \in[m, M]$;

- a unique mass market equilibrium where firm $i$ charges $R_{i}^{m}$ and $S_{i}^{m}$ satisfying (8)-(9) if and only if $\mu \in\left(\frac{c-\rho \cdot m}{1-\rho}, \infty\right)$.

The sufficient and necessary condition for the existence of the mass market equilibrium requires that the value of the new product market, $\mu$, is sufficiently high. We note that the condition now differs from the standard definition of a mass market (Bergemann and Välimäki, 2006; Ivanov, 2009), which requires $\mu \geq c$ (note that $c \geq \frac{c-\rho \cdot m}{1-\rho}$ ). We continue refer to the equilibrium as the "mass market equilibrium" as in Section 3, because it shares the feature that the marginal repeat consumer strictly prefers to stay in the new market rather than return to the outside option.

According to the sufficient and necessary condition for the niche market equilibrium when $\rho \in(0,1)$, we have $\mu \leq c \cdot \frac{1-\rho \cdot M / c}{1-\rho}<c<M$, suggesting that the majority of customers (i.e., more than $F(\mu)=\frac{1}{2}$ of consumers) leaves the market. Similarly, the sufficient and necessary condition for the semi-niche market equilibrium when $\rho \in(0,1)$ gives $\mu \leq c \cdot \frac{1-\rho \cdot R_{i}^{s} / c}{1-\rho}<c<R_{i}^{s}$, again suggesting that the majority of customers leaves the market.

As in the $\rho=0$ case, when $\rho>0$, second-period prices do not depend on first-period market share and firms' market power among repeat consumers is also greater in the semi-niche than in the mass market equilibrium, and greater still in the niche market equilibrium, because $R_{i}^{m}<R_{i}^{s}=R_{i}^{n}$. To see why, note that $R_{i}^{m} \leq m \leq R_{i}^{s} \leq M=R_{i}^{n}$, where the first inequality is verified by the proof of Proposition 2. Firms' market power among switching customers is

\footnotetext{
24 Because switching yields the marginal repeat customer being $\theta_{i}^{R S}$ where $\left[\rho \theta_{i}^{R S}+(1-\rho) \mu\right]-$ $[\rho m+(1-\rho) \mu]=0$.
} 
once more greatest in the mass market equilibrium. It is weakened in the semi-niche and niche market equilibrium as there is no effective competition for switchers.

Figure 7 illustrates the range of $\mu$ corresponding to each second period equilibrium for both $\rho=0$ (top half of figure) and $\rho>0$ (lower half of figure).

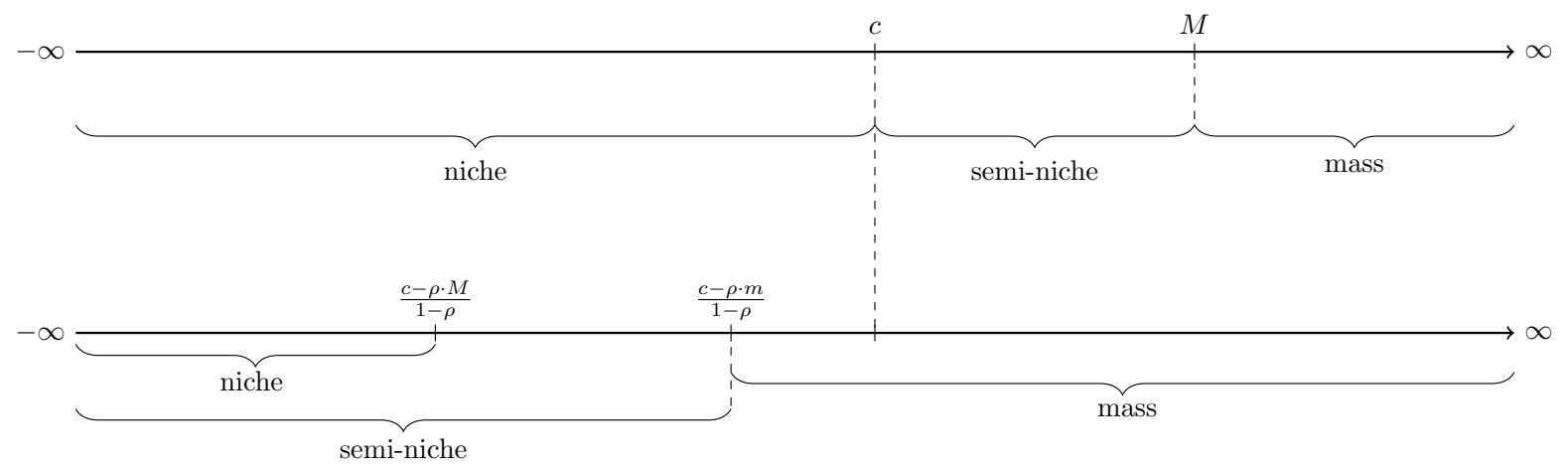

Figure 7: Relevant range of $\mu$ for each second-period equilibrium when $\rho=0$ (above) and when $\rho>0$ (below).

Another important observation is that firms actively compete for switchers only in the mass market equilibrium when $\rho>0$, whereas they compete for switchers in both the mass and seminiche market equilibrium when $\rho=0$. As illustrated by Figure 7, the mass market equilibrium when $\rho>0$ exists for a larger range of $\mu$ than the range for the mass and semi-niche market equilibrium when $\rho=0$. This suggests that firms compete more intensively for switchers in the second period when consumer valuations are positively correlated because they cannot secure a monopoly position in the repeat consumer market for $\mu \in\left(\frac{c-\rho m}{1-\rho}, c\right)$ when $\rho>0$, unlike when $\rho=0$. Corollary 2 below further illustrates that firms encounter more intensive second-period competition in the market of their existing customers when $\rho>0$ than when $\rho=0$.

Corollary 2. Denote by $R_{\rho=0}$ and $R_{\rho>0}$ the prices to repeat consumers in the mass and seminiche market equilibria given by Proposition 1 and 2, respectively. Then, it holds for all $\mu \geq c$ that $R_{\rho>0}<R_{\rho=0}$.

Proof. When $\mu>M$, firms compete in the mass market equilibrium in both $\rho=0$ and $\rho>0$ settings. Hence, $R_{\rho=0}=\frac{1}{2 f(\mu)}+c$, while $R_{\rho>0}$ is given by (8). Since $\theta_{i}^{R S}>\mu$ in the mass market when $\rho>0$, due to $R_{i}^{m}>S_{i}^{m}$, we have

$$
R_{\rho>0}=\frac{(1-\rho)\left[1-F\left(\theta_{i}^{R S}\right)\right]}{f\left(\theta_{i}^{R S}\right)}+c<\frac{1-\rho}{f(\mu)}+c<\frac{1}{2 f(\mu)}+c=R_{\rho=0} .
$$

When $\mu \in[c, M]$, firms compete in the semi-niche market equilibrium if $\rho=0$ but mass market equilibrium if $\rho>0$. Since the proof of Proposition 2 has shown that $R_{\rho>0}<m$, we know that $R_{\rho>0}<M=R_{\rho=0}$ due to $m<M$. 
Proposition 2 allows us to compare the second-period prices offered to repeat and switching consumers.

Corollary 3. In the mass market equilibrium when $\rho \in(0,1), R_{i}^{m} \geq S_{i}^{m}$; in the semi-niche market equilibrium, $R_{i}^{s}>S_{i}^{s}$.

Proof of Corollary 3. The first part is shown in the proof of Proposition 2, step 1. The second part is due to $R_{i}^{s} \geq m>c \geq S_{i}^{s}$, where the last inequality is due to $\mu \leq \frac{c-\rho \cdot \iota}{1-\rho} \leq \frac{c-\rho \cdot R_{i}^{s}}{1-\rho}$, hence, $\rho \cdot R_{i}^{s}+(1-\rho) \mu \leq c$.

Corollary 3 illustrates that firm $i$ price discriminates against their repeat consumers in the mass market equilibrium when $\rho \in(0,1)$, unlike when $\rho=0$ where firms set the same price for both groups. Repeat consumers have learned that their value for good $i$ is high enough to prevent switching to the untried product.

Note also that both the prices in the mass market equilibrium with $\rho>0$ converge to those with $\rho=0$.

Corollary 4. $\lim _{\rho \rightarrow 0} R_{i}^{m}=\lim _{\rho \rightarrow 0} S_{i}^{m}=\frac{1}{2 f(\mu)}+c$.

Proof. When $\rho>0$, we have $S_{i}^{m}<\mu$. Hence, $\theta_{-i}^{S O} \rightarrow-\infty$ when $\rho \rightarrow 0$, implying $S_{i}^{m} \rightarrow \frac{F\left(\theta_{-i}^{R S}\right)}{f\left(\theta_{-i}^{R S}\right)}+$ $c$. On the other hand, $R_{i}^{m} \rightarrow \frac{1-F\left(\theta_{-i}^{R S}\right)}{f\left(\theta_{-i}^{R S}\right)}+c$. Then, $\theta_{i}^{R S}=\mu$ must hold because $\theta_{i}^{R S}=\mu+\frac{R_{i}^{m}-S_{-i}^{m}}{1-\rho}$ only holds when $\theta_{i}^{R S}=\mu$. This implies that $\lim _{\rho \rightarrow 0} R_{i}^{m}=\lim _{\rho \rightarrow 0} S_{i}^{m}=\frac{1}{2 f(\mu)}+c$.

Recall that the $\rho=0$ setting is a special case of $\rho \leq 0$, which we analyze in Appendix C. Corollary 4 therefore states that the mass market equilibrium prices in the $\rho \in(0,1)$ setting converge to the mass market equilibrium prices in the $\rho=0$ setting.

Appendix D derives the own- and cross-price elasticities for repeat and switching consumer demand in each of the three equilibria. When $\rho=0$, a comparison between the mass and niche market cases is insightful. In the mass market case, the own-price elasticities to repeat and switching customers, respectively, are $E_{R_{i}^{m}}^{D_{i}^{R}}=-\frac{R_{i}^{m}}{R_{i}^{m}-c}$ and $E_{S_{i}^{m}}^{D_{i}^{S}}=-\frac{S_{i}^{m}}{S_{i}^{m}-c}$. Because $R_{i}^{m} \geq S_{i}^{m}$ when $\rho \in(0,1)$ (Corollary 3), the Lerner index measure of market power, $\frac{R_{i}^{m}-c}{c}$, is always greater in the repeat consumer market segment than in the switching consumer segment, $\frac{S_{i}^{m}-c}{c}$. Prices and market power in both consumer segments vary with $\rho$ and converge in both the $\lim _{\rho \rightarrow 0}$ (Corollary 4) and when $\rho=1$. As in the $\rho=0$ case, market power and markups are always greater in the niche market equilibrium than in the mass market equilibrium.

The relationships between $\rho$ and market power in each consumer segment reflect the trade-off arising from the fact that $\rho$ determines the extent of competition between the two products and also the information that consumers use to derive expected value for the rival product. In the 
niche market equilibria, the own-price elasticity for repeat consumers is $E_{R_{i}^{n}}^{D_{i}^{R}}=-\frac{M}{M-c}$, which gives a Lerner index of $\frac{M-c}{c}$, and there are no switching customers in equilibrium. The firm always has greater market power in the niche market than in the mass market, and markups in the niche market equilibrium are independent of $\rho$.

Of particular interest is the mass market equilibrium second-period profit from repeat consumers and switching consumers because their relative magnitude determines the intensity of first-period price competition. The second-period profits each firm can make from repeat customers and switching consumers are given, respectively, by: ${ }^{25}$

$$
\begin{aligned}
\pi_{i}^{R m} & =\frac{(1-\rho)\left[1-F\left(\theta_{i}^{R S}\right)\right]^{2}}{f\left(\theta_{i}^{R S}\right)} \\
\pi_{i}^{S m} & =\frac{\rho(1-\rho)\left[F\left(\theta_{-i}^{R S}\right)-F\left(\theta_{-i}^{S O}\right)\right]^{2}}{\rho f\left(\theta_{-i}^{R S}\right)+(1-\rho) f\left(\theta_{-i}^{S O}\right)} .
\end{aligned}
$$

Recall that when $\rho=0, R_{i}^{m}=S_{i}^{m}$ and the profits from repeat and switching customers are equal. When $\rho \in(0,1)$, however, although $R_{i}^{m} \geq S_{i}^{m}$, the relative magnitudes of the profits from repeat and switching customers in the mass market equilibrium cannot be ordered, and depend on demand-related parameters, including $\rho$. Note, however, that both profits converge to zero as $\rho$ converges to 1 .

Corollary 5. In the second period with $\rho \in(0,1)$ and suppose all consumers have purchased one of the goods,

- firm i's profit in the niche market equilibrium is given by $\lambda_{i} \pi(M)$;

- firm i's profit in the semi-niche market equilibrium is given by $\lambda_{i} \pi\left(R_{i}^{s}\right)$, where $R_{i}^{s} \in$ $[m, M]$

- firm i's profit in the mass market equilibrium consists of $\lambda_{i} \pi_{i}^{R m}$ from repeat consumers and $\lambda_{-i} \pi_{i}^{S m}$ from switching consumers.

\section{Sub-game perfect equilibria of the two-stage pricing game with $\rho \geq 0$}

Consumers make their first-period choice between the two goods and the outside option by comparing the following consumer surplus for $i=A, B$ and zero, given second-period equilibrium

\footnotetext{
${ }^{25}$ Section 5 provides numerical examples for the mass market equilibrium with $\rho>0$.
} 
prices $R_{i}^{*} \in\left\{R_{i}^{m}, R_{i}^{s}, R_{i}^{n}\right\}$ and $S_{-i}^{*} \in\left\{S_{-i}^{m}, S_{-i}^{s}, S_{-i}^{n}\right\}$ :

$$
\begin{aligned}
C S_{\rho \geq 0}=\underbrace{\left(\mu-p_{i}\right)}_{\text {consumer surplus from trying } i}+\delta \underbrace{\int_{\max \left(\theta_{i}^{R S}, \theta_{i}^{R O}\right)}^{\infty}\left(x-R_{i}^{*}\right) d F(x)}_{\text {consumer surplus from sticking with } i} \\
+\delta \underbrace{\max \left(0, \int_{\theta_{i}^{S O}}^{\theta_{i}^{R S}}\left[E\left(\theta_{-i} \mid x\right)-S_{-i}^{*}\right] d F(x)\right)}_{\text {consumer surplus from switching to }-i \text { or to the outside option }}
\end{aligned}
$$

and where $p_{i} \in\left\{p_{i}^{m}, p_{i}^{s}, p_{i}^{n}\right\}$ is the first-period price depending on whether the second-period equilibrium is mass, niche, or semi-niche.

If the consumer surplus from purchasing good $i$ is greater than from good $-i$, then firm $i$ 's first period market share $\lambda_{i}=1$. If the surplus from purchasing the goods is the same, then $\lambda_{i} \in[0,1]$. Otherwise, $\lambda_{i}=0$. Equation (5) shows how $\lambda_{i}$ affects firm $i$ 's second-period profit. Note that the second-period prices in each of the three equilibria are independent of $\lambda_{i}$, and the threshold cutoff values for switching and exiting consumers given in Definitions (1) to (3) are also independent of $\lambda_{i}$.

\subsection{No information spillovers, i.e. $\rho=0$}

The niche market equilibrium applies for all new product markets that are minor innovations, i.e., whenever $\mu<c$, according to Proposition 1. In these markets, first-period prices are such that firms make no profits.

Proposition 3 (Zero profit equilibrium). Suppose $\mu-c<0$ and $\rho=0$. If and only if $\mu \in$ $[c-\delta \cdot S S(M), c)$, there exists an equilibrium in which firm $i \in\{A, B\}$ makes zero profit by charging $p_{i}^{n}=c-\delta \pi(M)$ in the first period and charging $R_{i}^{n}=M, S_{i}^{n} \geq c$ in the second-period niche market equilibrium given by Proposition 1.

When $\mu-c$ is sufficiently low, none of the consumers find it worthwhile to switch even though each firm charges the monopoly price to its repeat purchasers. Firms therefore compete intensely in the first period for market share. They discount the first-period price below marginal cost to the point where they make a loss in the first period that exactly offsets second-period profits and make zero profits overall. ${ }^{26}$ The lower bound of innovation value $-\delta \cdot S S(M)$ guarantees that consumer surplus $C S_{\rho \geq 0}$ is non-negative. Note also that since buying only in the second

\footnotetext{
${ }^{26}$ In such an equilibrium, consumers obtain all the social surplus. This is precisely why the existence of the equilibrium requires $\mu \geq c-\delta \cdot S S(M)$. The condition is equivalent to $(\mu-c)+\delta \cdot S S(M) \geq 0$, in which the first term on the LHS is the social surplus from the first-period and the second term is discounted social surplus from the second-period. Since firms make zero profit in the equilibrium, according to the proposition, consumer surplus equals total social surplus, i.e., the LHS of the condition. The condition thus guarantees consumer surplus is non-negative which is necessary for the equilibrium to exist.
} 
period yields a consumer surplus of $\mu-S_{i}^{n}<0$ - assuming firms treat any new consumers as switchers regardless of whether they switch from the old technology or the rival good-all consumers purchase in the first period.

In contrast, firms make positive profits whenever the second-period equilibrium is either the mass market or the semi-niche market equilibria. According to Proposition 1, one of these equilibria apply when the new product market is sufficiently valuable, that is, it represents a major innovation, with $\mu \geq c$. We characterize the relevant first-period equilibrium and provide sufficient and necessary conditions for its existence in Proposition 4.

Proposition 4 (Positive profit equilibria). When $\mu-c \geq 0$ and $\rho=0$ :

- if and only if $\mu>M$, there exists an equilibrium in which firm $i \in\{A, B\}$ makes a profit of $\frac{\delta}{4 f(\mu)}>0$ by charging $p_{i}^{m}=c$ in the first period and charging $R_{i}^{m}=S_{i}^{m}=\frac{1}{2 f(\mu)}+c$ in the second-period mass market equilibrium given by Proposition 1;

- if and only if $c \leq \mu \leq M$, there exists an equilibrium in which firm $i \in\{A, B\}$ makes a profit of $\delta(\mu-c) F(M)>0$ by charging $p_{i}^{s}=c-\delta[\pi(M)-(\mu-c) F(M)]$ in the first period and charging $R_{i}^{s}=M, S_{i}^{s}=\mu$ in the second-period semi-niche market equilibrium given by Proposition 1.

In both these cases, the intensity of first-period price competition is determined by the relative profits from selling to switching and repeat customers in the second period. When the equilibrium features the semi-niche equilibrium in the second period, according to Corollary 1 , firms earn greater profit from repeat customers, and therefore set a first-period price lower than the marginal cost $c$, but not low enough to offset all their future profits. When the equilibrium features the mass market equilibrium in the second period, firms make exactly the same profit from repeat and switching consumers in the second period, as shown in Corollary 1. Neither firm is willing to discount the price below marginal cost in the first period to attract customers to earn profit in the future. The first-period resembles Bertrand competition and firms set a first-period price that equals the marginal cost $c$.

\subsection{With Information Spillovers, i.e. $\rho \in(0,1)$}

When the relevant second-period equilibrium is either the niche or semi-niche equilibrium, which apply when the new market value is low, as shown in Proposition 2, firms make zero profits in the two-stage pricing game. Proposition 5 below shows this result.

Proposition 5 (Zero profit equilibria). When $\rho \in(0,1)$ : 
- if and only if $\mu \in\left[c-\delta \cdot S S(M), \frac{c-\rho \cdot M}{1-\rho}\right)$, there exists an equilibrium in which firm $i=$ $A, B$ makes a profit of zero by charging $p_{i}^{n}=c-\delta \pi(M)$ in the first period and charging $R_{i}^{n}=M, S_{i}^{n} \geq c$ in the second-period niche market equilibrium given by Proposition 2;

- if and only if $\mu \in\left[c-\delta \cdot S S(\iota), \frac{c-\rho \cdot \iota}{1-\rho}\right]$, where $\iota \in[m, M]$, there exists a continuum of equilibria in which firm $i=A, B$ makes a profit of zero by charging $p_{i}^{s}=c-\delta \pi\left(R_{i}^{s}\right)$ in the first period and charging $R_{i}^{s} \in[m, \iota], S_{i}^{s}=\rho R_{i}^{s}+(1-\rho) \mu$ in the second-period semi-niche market equilibria given by Proposition 2.

In Proposition 5, the lower bounds of the sufficient and necessary conditions for the equilibria, i.e. $c-\delta \cdot S S(M)$ and $c-\delta \cdot S S(\iota)$, ensure that the consumer surplus over the two periods is non-negative. For the second equilibrium, observe that $c-\delta \cdot S S(\iota)$ is increasing in $\iota$, whereas $\frac{c-\rho \cdot \iota}{1-\rho}$ is decreasing in $\iota$. When $\iota=M$, there exists a continuum of equilibria in which the second-period price $R_{i}^{s}$ takes any value in $[m, M]$ and $S_{i}^{s}=\rho R_{i}^{s}+(1-\rho) \mu$, if and only if $\mu \in\left[c-\delta \cdot S S(M), \frac{c-\rho \cdot M}{1-\rho}\right]$. This range of $\mu$ coincides with the sufficient and necessary condition for the first equilibrium. Hence, both equilibria exist in such an interval and yield profits of zero.

We now turn to the mass market equilibrium when $\rho \in(0,1)$, which Proposition 2 shows applies for a large range of new market values, for some minor innovations, as well as for all major innovations. The following proposition shows that firms make positive profits in the twostage pricing game whenever the mass market equilibrium applies in the second period when $\rho \in(0,1)$.

Proposition 6 (Positive profit equilibrium). Suppose $\rho \in(0,1)$. If and only if $\mu \geq \max \left(c-\delta\left(S S^{m}-2 \pi_{i}^{S m}\right)+\max \left(0, \delta\left(\mu-S_{i}^{m}\right)\right), \frac{c-\rho \cdot m}{1-\rho}\right)$, there exists an equilibrium in which firm $i=A, B$ makes a profit of $\delta \pi_{i}^{S m}>0$ by charging $p_{i}^{m}=c-\delta\left(\pi_{i}^{R m}-\pi_{i}^{S m}\right)$ in the first period and charging $R_{i}^{m}, S_{i}^{m}$ in the second-period mass market equilibrium, where $R_{i}^{m}, S_{i}^{m}, \pi_{i}^{R m}$, and $\pi_{i}^{S m}$ are given by (8), (9), (11), and (12), respectively, and

$$
S S^{m}=\int_{\theta_{i}^{R S}}^{\infty}(x-c) d F(x)+\int_{\theta_{i}^{S O}}^{\theta_{i}^{R S}}\left[E\left(\theta_{-i} \mid x\right)-c\right] d F(x) .
$$

Proposition 6 shows that the value of the innovation must be sufficiently large to guarantee the existence of a profitable equilibrium. However, when $\mu<c$ and $S S^{m}>2 \pi_{i}^{S m}$ hold, then $c-\delta\left(S S^{m}-2 \pi_{i}^{S m}\right)+\max \left(0, \delta\left(\mu-S_{i}^{m}\right)\right)<c$, which implies that firms make positive profits even in some new markets where the value for the average consumer is below marginal cost, 
that is, for some minor innovations. However, when $\rho=0$, there are switching consumers and, hence, positive profits only for major innovations.

Corollary 6. If $\rho \in(\bar{\rho}, 1)$, a mass market equilibrium exists for minor innovations $\mu \in$ $\left[\max \left(c-\delta \cdot\left(S S^{m}-2 \pi_{i}^{S m}\right), \frac{c-\rho \cdot m}{1-\rho}\right), c\right)$, where $\bar{\rho} \equiv \sup \left\{\rho \mid S S^{m}=2 \pi_{i}^{S m}\right\}$ and $\bar{\rho}=0$ if $S S^{m}>$ $2 \pi_{i}^{\text {Sm }}$ for all $\rho \in(0,1]$.

Proof. Given that $\mu<c$, we have $\max \left(0, \delta\left(\mu-S_{i}^{m}\right)\right)=0$. When $S S^{m} \leq 2 \pi_{i}^{S m}$, the sufficient and necessary condition in Proposition 6 reduces to $\mu \geq c-\delta\left(S S^{m}-2 \pi_{i}^{S m}\right)$. However, our discussion for the $\rho=1$ case in Section 2, as well as the fact that $\pi_{i}^{S m}$ converges to zero as $\rho$ approaching 1, imply that for $\rho$ sufficiently close to 1 , it must be true that $S S^{m}>2 \pi_{i}^{S m}$. Therefore, as long as the correlation $\rho$ is no less than the highest $\rho$ such that $S S^{m}=2 \pi_{i}^{S m}$, i.e., $\bar{\rho} \equiv \sup \left\{\rho \mid S S^{m}=2 \pi_{i}^{S m}\right\}$, we must have $\max \left(c-\delta \cdot\left(S S^{m}-2 \pi_{i}^{S m}\right), \frac{c-\rho \cdot m}{1-\rho}\right)<c$, which suggests some minor innovations enable firms to make positive profits.

Proposition 6 reinforces the general finding that the intensity of first-period price competition depends on the relative profitability of repeat and switching consumers in the second period. When the mass market equilibrium applies in the second period and $\rho \in(0,1)$, these profits are given in equations (11) and (12), respectively. First period prices can be below, above, or equal to marginal cost.

Figure 8 presents a numerical simulation for a set of model parameters that produces a mass market second-period equilibrium when there are information spillovers, i.e. $\rho \in(0,1)$. The left hand side panel plots second-period profits from repeat and switching consumers and shows that the latter exceed the former at lower values of $\rho$, given chosen values of $c, \sigma$, and $\mu$. The right hand side panel plots the first-period price relative to the marginal cost of zero. For values of $\rho$ where the profits from switching consumers exceed those from repeat consumers, first-period price exceeds marginal cost. Therefore in this numerical simulation, for lower values of $\rho$ in $\rho \in(0,1)$, firms make positive profits in both the first and second periods.

\section{Discussion of the role of information spillovers}

\subsection{Information spillovers result in duopoly profits in less-valuable new prod- uct markets.}

Our equilibrium analysis shows that the presence of positive information spillovers, $\rho \in(0,1)$, increases the range of new market values over which firms make profits compared to when $\rho=0$. The comparative statics w.r.t. innovation value, based on our analysis given in Propositions 3 - 

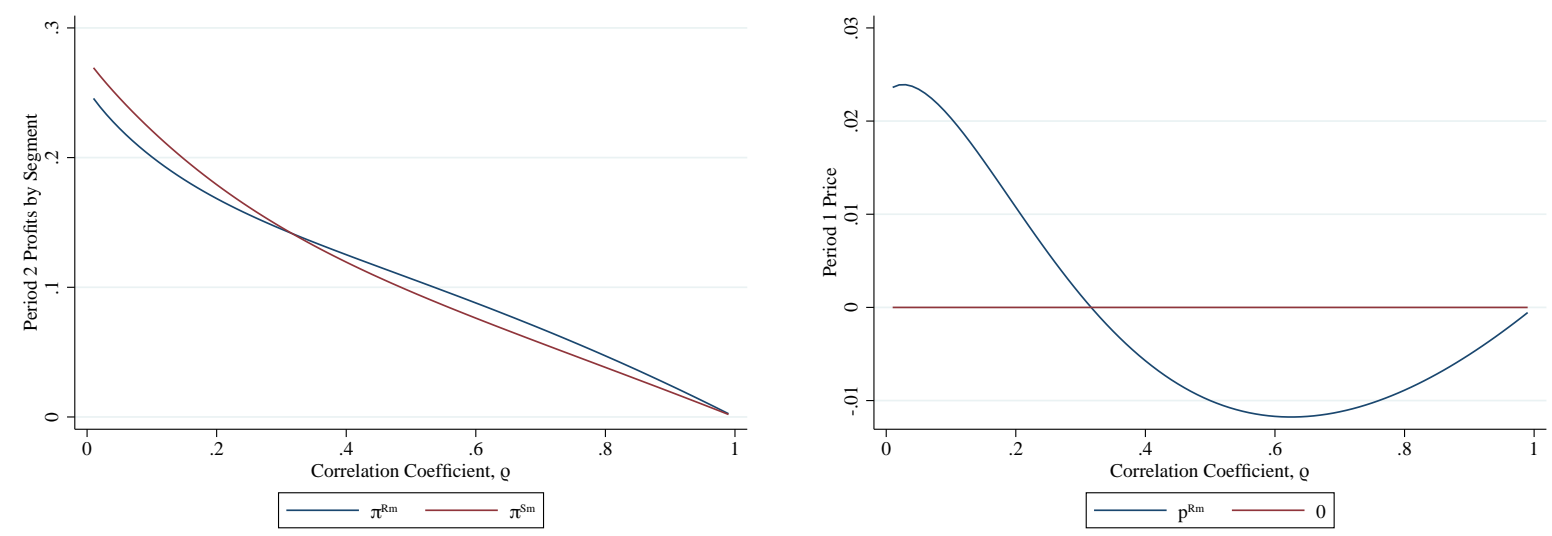

Figure 8: For lower values of $\rho>0$, we have $\pi_{i}^{R m}<\pi_{i}^{S m}$ and hence, $p_{i}^{m}>c$, where $\mu=\sigma=0.5$ and $c=0$. Note that $R_{i}^{m}>S_{i}^{m}$ still holds.

6, are summarized in Figure 9, which extends Figure 7 to reflect the cutoffs for the two-period game.

The figure has two parts, and in each case the axis varies $\mu$ holding $c$ constant. The top half of the figure considers the case of $\rho=0$, and provides the intervals of $\mu$ that are sufficient and necessary for the existence of equilibria, showing also the second-period equilibria for the relevant range. The lower half of the figure does the same for when $\rho>0$. Note that we focus on the situation illustrated by Corollary 6, i.e., $\rho \in(\bar{\rho}, 1)$. Our discussion following Figure 9 applies to all $\rho>0$.

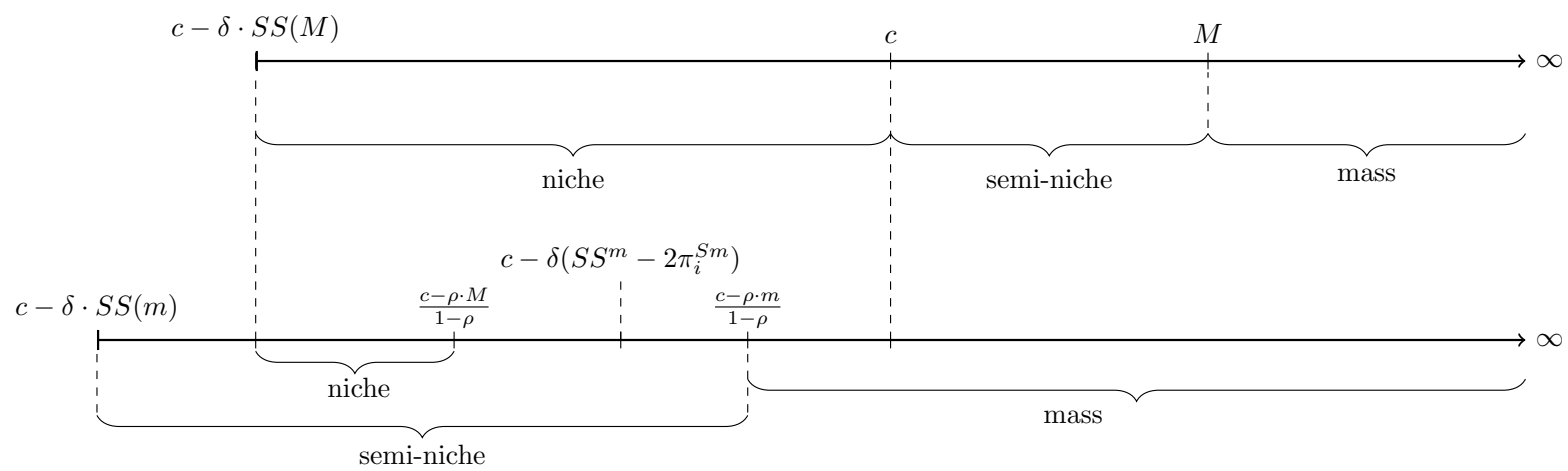

Figure 9: Equilibrium and innovation: on top is the range of innovation $\mu$ corresponding to different equilibria for $\rho=0$ and below for $\rho \in(\bar{\rho}, 1)$.

Two aspects of Figure 9 merit particular focus. First, relating to the far left hand side of both halves of the figure, when $\mu \in[c-\delta \cdot S S(m), c-\delta \cdot S S(M)]$ if $\rho>0$, there exist some seminiche market equilibria only if $\rho>0$. No equilibrium exists for this range of $\mu$ in which firms enter the market in the first period if $\rho=0$. Hence, positive spillovers ensure an equilibrium exists for these minor innovations.

Second, we turn to minor innovations where $\mu \in\left(\max \left(c-\delta\left(S S^{m}-2 \pi_{i}^{S m}\right), \frac{c-\rho \cdot m}{1-\rho}\right), 0\right)$. Recall 
from Corollary 6 that the lower bound of the condition is below zero when $\rho \in(\bar{\rho}, 1)$. These innovations yield positive profits for the firms only when there are positive information spillovers. This can be seen by noting that firms earn positive profits in mass market equilibria, but zero profits in niche market equilibria. Comparing the top and bottom part of the figure reveals how mass market equilibria result over an interval of innovation values with a smaller lower bound when $\rho \in(\bar{\rho}, 1)$. This result implies that when the extent of exogenous industry innovation is sufficiently low, firms will prefer to produce similar products to ensure positive profits.

Figure 10 plots firms' profit as a function of $\mu$, when the marginal cost is fixed at $c=0.5$. The blue line is each firm's profit when $\rho>0$ (in this case $\rho$ is fixed at 0.5 ) and the red line is the profit when $\rho=0$. Here, minor innovations with $\mu \in\left(\max \left(c-\delta\left(S S^{m}-2 \pi_{i}^{S m}\right), \frac{c-\rho \cdot m}{1-\rho}\right), c\right)$ correspond to $\mu \in[0.2,0.5]$. When $\mu$ is sufficiently high, firms strictly prefer $\rho=0$. When $\mu$ lies below $c=0.5$, firms make positive profits only if $\rho>0$, illustrating that firms strictly prefer $\rho>0$ when the extent of innovation is sufficiently low.

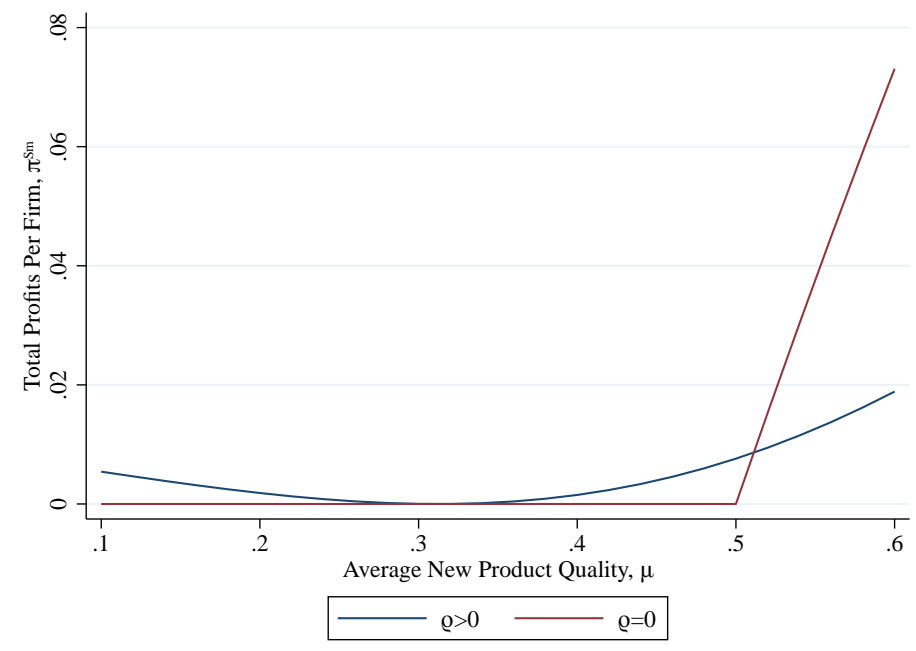

Figure 10: An example with $c=\sigma=\rho=0.5$. Each firm's profit given $\rho>0$ (blue) is positive when $\mu$ is less than $c=0.5$, whereas the profit given $\rho=0$ (red) is zero when $\mu-c \leq 0$.

\subsection{Switching consumers guarantee firm profits.}

Our analysis so far illustrates that each firm's total discounted profit in the game can never be more than the profit the firm would make if it had zero market share in the first period and its competitor had a market share of one. To see why, recall from Propositions 3 to 6 that firms make zero profit over the two periods if no consumers switch in the second-period equilibrium. In all equilibria where firms make profits, the equilibrium first-period price is low enough that the cost of gaining market share in the first period is equal to the relative profitability of a repeat consumer in the second period compared to a switching consumer. 
Theorem 1 below shows that this intuition applies in general to any symmetric equilibrium in the game for all $\rho \in[-1,1]$, regardless of the form of equilibrium in the second period.

Let $\lambda_{i} \pi^{R *}$ be firm $i$ 's second-period profit from repeat consumers and $\lambda_{-i} \pi^{S *}$ be firm $i$ 's second-period profit from switching consumers in any symmetric equilibrium.

Theorem 1. For any $\rho \in[-1,1]$, firm $i$ 's first period price in any symmetric equilibrium with any market share $\lambda_{i} \in[0,1]$ is given by $p^{*}=c-\delta\left(\pi^{R *}-\pi^{S *}\right)$ and its profit in the two-stage pricing game is given by $\delta \pi^{S *}$.

Proof. Since the goods are ex ante homogeneous from the consumers' perspective and we focus on symmetric equilibria, consumers purchase the cheaper product in the first period. Suppose $\pi^{R *}>\pi^{S *}$, that is, firm $i$ benefits from increasing first-period market share. Then, both firms compete for first period market share by lowering the first period price.

If firm $i$ deviates by charging a slightly lower price than $p^{*}$, it then gains all the rival firm's market share $\lambda_{-i}$ and incurs a cost of $\lambda_{-i}\left(c-p^{*}+\epsilon\right)$ in the first period, where $\epsilon>0$. Since the share $\lambda_{-i}$ of consumers are now firm $i$ 's repeat consumers in the second period, it makes an additional profit from repeat consumers, $\lambda_{-i} \pi^{R *}$, but forgoes the profit that it would have made from switching consumers, $\lambda_{-i} \pi^{S *}$. In other words, the net benefit in the second period is given by $\lambda_{-i}\left(\pi^{R *}-\pi^{S *}\right)$. Since $p^{*}=c-\delta\left(\pi^{R *}-\pi^{S *}\right)$, and hence, $\lambda_{-i}\left(c-p^{*}+\epsilon\right)=$ $\delta \lambda_{-i}\left(\pi^{R *}-\pi^{S *}\right)+\lambda_{-i} \epsilon>\delta \lambda_{-i}\left(\pi^{R *}-\pi^{S *}\right)$, the cost is greater than the discounted benefit. The deviation is thus not profitable.

If firm $i$ deviates by charging a higher price than $p^{*}$, it gives up $\lambda_{i}$ market share to the rival firm. Hence, its first-period profit increases by $\lambda_{i}\left(c-p^{*}\right)$, as it avoids this loss. In the second period, it makes an additional profit from switching consumers, $\lambda_{i} \pi^{S *}$, but forgoes any profit from repeat consumers, $\lambda_{i} \pi^{R *}$. Hence, the net loss in the second period is given by $\lambda_{i}\left(\pi^{R *}-\pi^{S *}\right)$. Since $p^{*}=c-\delta\left(\pi^{R *}-\pi^{S *}\right)$, we know that the first-period benefit is equal to the second-period loss. The deviation is thus not profitable.

In fact, the above proof for the case when $\pi^{R *}>\pi^{S *}$ also applies to $\pi^{R *} \leq \pi^{S *}$. In this case, firms compete for a smaller first-period market share by increasing the first-period price up to a point where the additional profit earned in the first period is balanced by the additional loss in the second period. Note that in this case, $p^{*} \geq c$.

The proof so far applies to any $\lambda_{i} \in[0,1]$ such that $\lambda_{i}+\lambda_{-i}=1$. This means that it is not profitable for a firm to deviate by selling only in the second period while the rival firm sells in both periods. In such a situation, the firm makes a profit of $\delta \pi^{S *}$ which equals the equilibrium profit. Alternatively, when the rival firm sells only in the second period, firm $i$ makes zero profit by selling only in the second period. Therefore, selling only in the second period is weakly 
dominated by selling in both periods.

To calculate firm $i$ 's profit, substituting for $p^{*}$ in the profit function gives:

$$
\pi_{i}^{*}=\lambda_{i}\left(p^{*}-c\right)+\delta\left(\lambda_{i} \pi^{R *}+\lambda_{-i} \pi^{S *}\right)=\delta \pi^{S *}
$$

Hence, each firm's profit in the two-stage pricing game in any symmetric equilibrium is equal to the discounted second-period profit it would earn had it had zero market share in the first period.

The proof of Theorem 1 relies on the following simple derivations. If firm $i$ deviated to a lower price, it would capture the rival firm's share $\lambda_{-i}$. It then makes an additional profit from this share of (repeat) consumers, but has to give up profit from switching consumers whose share is exactly $\lambda_{-i}$. The price $p^{*}$ given in the theorem balances the first-period incremental profit and the discounted second-period incremental profit. In any symmetric equilibrium with any $\lambda_{i}$, firm $i$ 's profit in the whole game is given by $\lambda_{i}\left(p^{*}-c\right)+\delta\left(\lambda_{i} \pi^{R *}+\lambda_{-i} \pi^{S *}\right)=\delta \pi^{S *}$. Note that the first-period price depends on the relative magnitude of profits from repeat and switching consumers.

Further intuition for Theorem 1 comes from a comparison to the monopoly case, where the theorem does not apply. In the mass market equilibrium and over a large range of parameter values in the niche and semi-niche equilibria, the monopolist earns profits by setting all prices at least as high as in the duopoly case. In duopoly, the second-period differential profits from repeat rather than switching consumers are competed away via low first-period price, and this mechanism is missing in the monopoly case. First-period prices need to be low enough only to ensure all consumers purchase in the first period. The monopolists' total profits are not therefore limited to an amount equivalent to the surplus it could extract from switching consumers. ${ }^{27}$

Theorem 1 does, however, generalize to other demand structures such as the binary consumer type duopoly case, where consumers have either a high or low value for both products. ${ }^{28}$ In Appendix C, we show that firms can always make a positive profit in the continuous consumer type case studied here in any market equilibrium when $\rho<0$. This is due to the fact there are always some consumers who switch brand in the second period in this setting and Theorem 1 applies. $^{29}$

\footnotetext{
${ }^{27}$ In Appendix E, we show that firms can make greater profits when there is no informational switching cost, that is, when consumers know their valuations for all goods perfectly, than when there is such a cost. The intuition is that in the former setting, goods are more differentiated, softening competition, in the first period. Hence, as long as the firms are not too patient (small $\delta$ ) such that the first-period profit dominates firms' profit from the whole game, profits with no informational switching costs are greater.

${ }^{28} \mathrm{An}$ analysis of this case is available on request from the authors.

${ }^{29}$ In Appendix F, we study the impact of asymmetric willingness to pay in a setting where the marginal distri-
} 


\subsection{Profits increase in $\rho$ for some range of $\rho \in(0,1)$.}

Comparative statics regarding profits, consumer surplus, and social surplus w.r.t. $\rho$ are shown here in a numerical example with $c=\sigma=\mu=0.5$. The focus is on the mass market equilibrium when $\rho>0$, where profit is calculated in equation (12) and social surplus is calculated in equation (13). The consumer surplus is the difference between the social surplus and twice the profits (since there are two firms). The top left panel of Figure 11 shows that the profit firms
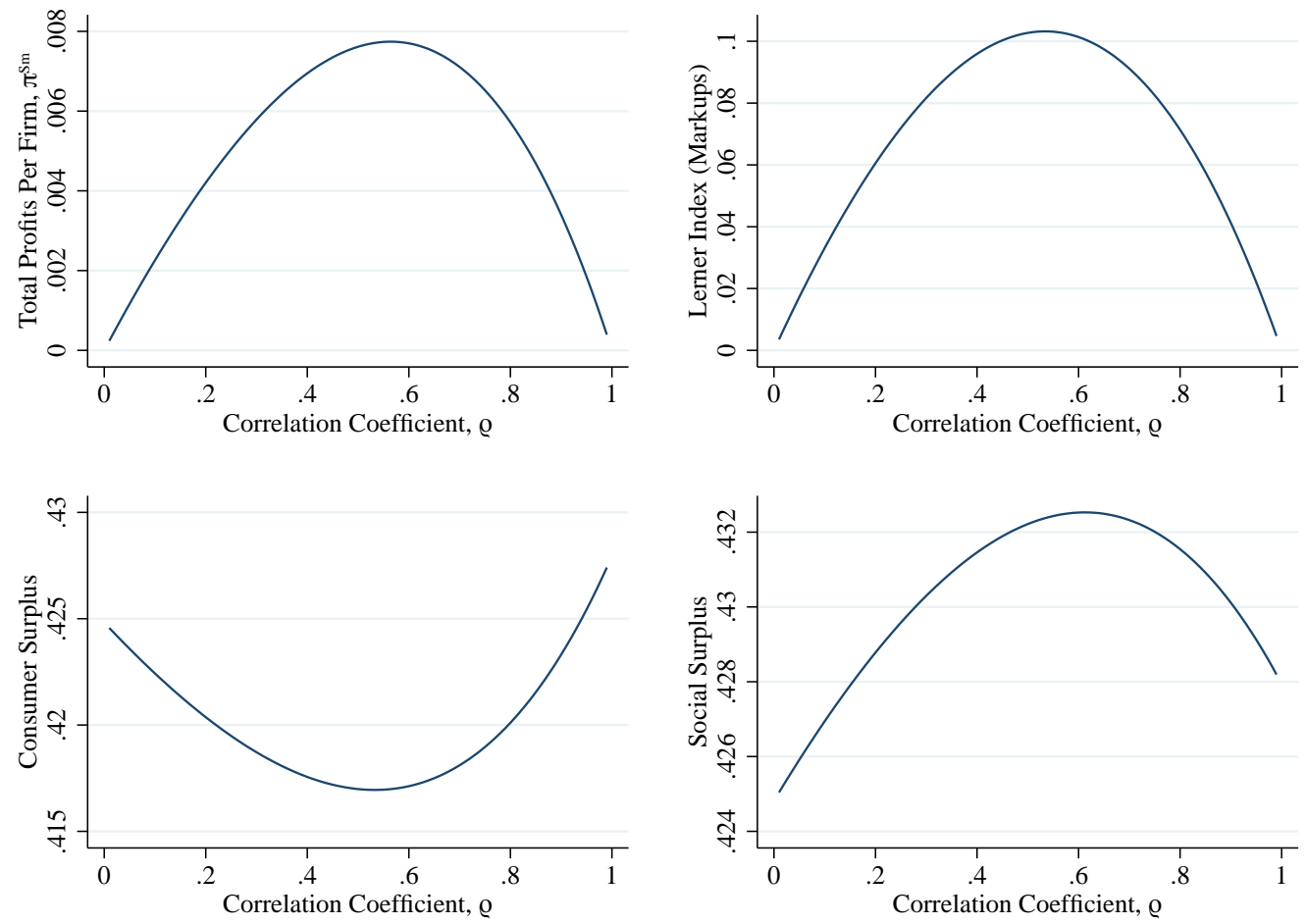

Figure 11: An example of profit, lerner index, consumer surplus, and social surplus as a function of $\rho$ in a mass market equilibrium, where $c=\sigma=\mu=0.5$.

make in the mass market equilibrium can be a non-monotonic function of $\rho$. With the chosen values of $c, \sigma$, and $\mu$, profit is maximized at around $\rho=0.6$.

Theorem 1 tells us that any differential profit earned from repeat consumers over profit from switching consumers is offset by first-period losses. Therefore, if total profits increase over a range of $\rho$, it must be that profits from switching consumers are increasing over that range. Because $\rho$ measures information spillovers as well as the degree of substitutability between products, it has two opposing effects on each firm's profits from switching consumers. Products become closer substitutes, and so prices and profits decrease in $\rho$. However, because consumers are now better informed about their value for competing products, the mass of 
switching consumers at equilibrium prices $S_{i}^{m} \leq R_{i}^{m}$ can be increasing in $\rho$. When the second effect dominates, total firm profits are increasing in $\rho$, and closer competition allows both firms to make greater profits. The trade off of these two effects is summarized in the top right hand side panel of the figure, presenting the Lerner Index for switchers, that is, the percentage

markup over marginal cost that is charged to them, $\frac{S_{i}-c}{c}$. Because the markup to repeat consumers, which is monotonically decreasing in $\rho$ is always offset by lower first-period prices, the non-monotonic markup to switchers delivers the non-monotonic overall profit function shown in the top left panel.

A comparison to the binary consumer type mass market equilibrium case helps illustrate how the non-monotonicity in profits arises in the continuous-type case. In the binary mass market case, a consumer's conditional expectation of $\theta_{i}$ after a bad experience with $i$ is always decreasing in $\rho$ meaning that switching demand becomes less elastic in $\rho$. In the continuous type mass market case, because some consumers leave the market after a bad experience, the conditional expectation of $\theta_{i}$ amongst potential switchers is always increasing in $\rho$, leading to increasing markups over some range.

The bottom left panel of Figure 11 shows that the consumer surplus over the two periods is U-shaped. Social surplus, given in the bottom right panel of Figure 11, has an inverse U shape, similar to firms' profits. Although the profit, consumer surplus, and social surplus are not necessarily U-shaped or inverse U-shaped, in general, it is true that profits increase and consumer surplus decrease in $\rho$ over some range of $\rho$.

\section{Conclusion}

This paper analyzes competition in duopoly new goods markets where demand has a novel feature: consumption is informative about a consumer's value for other similar goods as well as for the trialled good. This extends the definition of experience goods in Nelson (1970) to include within-consumer information spillovers from consumption. Information spills over when the new products are partial substitutes, that is, when a consumer's values for the two goods are positively correlated. Because consumers partially learn about non-trialled products, the informational switching cost (Klemperer, 1987a, 1995; Villas-Boas, 2006) locking consumers into the product they sampled is reduced.

One surprising result from the model is that such information spillovers allow firms to make positive profits in markets where the overall expected value of the new goods relative to preexisting alternatives is low. If consumer values were uncorrelated, each firm would be unable to make positive duopoly profits in these situations, but correlated learning across products 
expands the market for new goods to enable non-degenerate or non-monopoly structures.

The mechanism is that informative consumption experiences allow each firm to segment experienced consumer demand into their own repeat consumers and the consumers who switch to them after trialling the rival product. Firms are able to price discriminate between segments. Because firms compete in first-period prices for repeat consumers, anticipating the presence of profitable switchers softens dynamic price competition between the two firms. In some cases, switching consumers are more profitable than repeat consumers, and the equilibrium first-period price exceeds marginal cost. In these cases, firms have positive profits in both periods.

A second surprising result is that consumer surplus can be increasing, and firm profit decreasing, in the degree of product differentiation over some range. This paper, hence, shows that understanding how consumer choices respond to new products being imperfect substitutes is important for welfare analysis of different market structures. For the innovative experience goods studied here, our analysis shows consumers are not necessarily better off, and can be worse off, when products are closer substitutes. This arises due to the impact of learning through consumption on willingness to pay, together with firms' ability to price discriminate based on purchasing history.

If the game were extended to more periods, the set of possible purchasing histories would expand to include consumers who have trialled both products, in different orders, and consumers who have experienced only one product in prior periods. Firms' would find it optimal to price discriminate between all past purchasing histories. Although we have not found the equilibria of this longer game, we think it likely that price discrimination would continue to allow firms to gain some of the surplus from efficient consumer sorting and soften initial price competition, as firms would compete only for the differential profits between consumer groups. 


\section{Appendix}

\section{A An example of price discrimination based on past purchasing history in retail banking.}

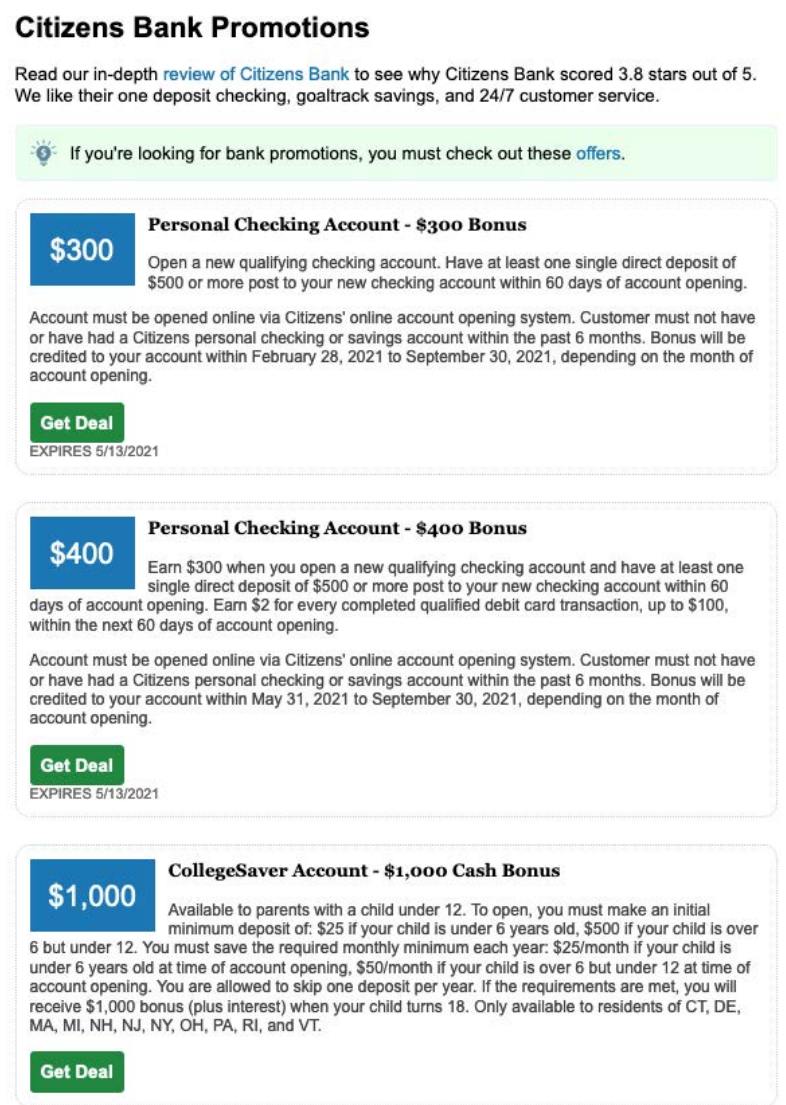

Figure A.1: Promotional offers to new Citizens Bank customers, available when opening a checking account, and varying with likely purchasing history.

The rest of the appendix contains: B. Proofs; C. Negative information spillovers; D. Price elasticities; E. Competitive pricing of normal goods: a benchmark; F. Asymmetric distribution; G. Second period equilibrium under uniform pricing.

\section{B Proofs}

Proof of Proposition 1. We consider each equilibrium in turn:

\section{Step 1: Niche market equilibrium}

In the niche market equilibrium, there are no switching customers. Each firm finds it optimal to charge the monopoly price $M$ to its repeat customers. Suppose firm $-i$ charges a price $S_{-i} \geq c$ to the customers of firm $i$. Then, the type $\theta_{i}=M$ has a surplus of $\mu-S_{-i} \leq \mu-c$ if she 
switches to $-i$. Then, a sufficient condition for the existence of the niche market equilibrium is that $\mu \leq c$, as type $\theta_{i}=M$ and all types with higher valuations must find it unprofitable to switch. Firm $-i$ makes zero profit from $i$ 's market in such an equilibrium, and deviating to any price below $c$ is not profitable.

Now we turn to necessity. Suppose the niche market equilibrium exists, then the customer with $\theta_{i}=M$ must find it unprofitable to switch to good $-i$, i.e., $\mu-S_{-i} \leq \theta_{i}-M=0$ for any $S_{-i} \geq c$, which implies that $\mu \leq c$.

Regarding uniqueness, note that in the niche market equilibrium firms do not face any competition in their own territory. Hence, each firm behaves as a monopoly. Therefore, there does not exist any other niche market equilibrium given that $\mu<c$. No mass or semi-niche market equilibria exist either when $\mu \leq c$, according to step 2 and 3 of this proof.

\section{Step 2: Semi-niche market equilibrium}

Firm $i$ makes monopoly profit in its own market and firm $-i$ makes a profit of $(\mu-c) F(M)$ in firm $i$ 's market. Since firm $-i$ charges price $\mu$, any customers of firm $i$ have a surplus of zero - equal to the value of the outside option - should they switch to firm $-i$. Hence, charging the monopoly price $M$ must be optimal for firm $i$ because its consumers are in effect choosing between purchasing from $i$ or the outside option.

According to the sufficient condition $\mu \geq c$, firm $-i$ makes non-negative profits. Firm $-i$ cannot make greater profits from increasing the price as then no customers from firm $i$ would switch. Suppose firm $-i$ decreases the price to $S<\mu$, then its profit from customers from firm $i$ is given by

$$
\int_{-\infty}^{\mu+M-S}(S-c) d F(x)=(S-c) F(\mu+M-S)
$$

By the first order derivative w.r.t $S$ :

$$
F(\mu+M-S)-(S-c) f(\mu+M-S)=F(\mu+M-S)\left[1-(S-c) \frac{f(\mu+M-S)}{F(\mu+M-S)}\right],
$$

we can find the optimal deviation price $S^{*}$ given by

$$
S^{*}=\frac{F\left(\mu+M-S^{*}\right)}{f\left(\mu+M-S^{*}\right)}+c .
$$

The monotone hazard rate property of the normal distribution guarantees that the above solu- 
tion is indeed the profit maximizer. Suppose $S^{*}<\mu$, then

$$
S^{*}>\frac{F(M)}{f(M)}+c \geq \frac{1}{2 f(\mu)}+c \geq \mu
$$

where the second and last inequalities are due to the sufficient condition $M \geq \mu$. This contradicts $S^{*}<\mu$. Thus, any deviation $S<\mu$ must be not more profitable for firm $-i$ than charging $S_{i}^{s}=\mu$. Alternatively, any deviation $S>\mu$ will not attract any switchers and hence, is dominated by charging $\mu$.

Now we turn to necessity. Given that the semi-niche equilibrium exists, it must be true that $\mu \geq c$ as otherwise firm $-i$ prefers to deviate to a higher price to avoid a loss. To prove that $\mu \leq M$ is also a necessary condition, suppose $\mu>M$. The fact that $S_{i}^{s}=\mu$ in equilibrium implies that $S^{*} \geq \mu$, where $S^{*}$ is given by (15). Otherwise, firm $i$ would prefer a price lower than $\mu$. But then $S^{*} \geq \mu$ implies that

$$
S^{*} \leq \frac{F(M)}{f(M)}+c<\frac{1}{2 f(\mu)}+c<\mu,
$$

which is a contradiction. Thus, $S^{*}<\mu$ when $\mu>M$, which implies that charging $\mu$ is not optimal for firm $-i$. This then contradicts the existence of the equilibrium.

Now we turn to uniqueness. Suppose there exists an alternative semi-niche market equilibrium where firm $i$ charges $\tilde{P}^{s n} \neq M$. Since both switching to firm $-i$ and choosing the outside option yield zero to consumers, firm $i$ 's price decision replicates that of a monopoly. Hence, it must hold that $\tilde{P}^{s n}=M$. Neither does there exist any mass or niche market equilibrium when $c \leq \mu \leq M$, according to steps 1 and 3 of this proof.

\section{Step 3: Mass market equilibrium}

For the mass market equilibrium, firm $i$ 's profit function is given by:

$$
\left(R_{i}-c\right) \cdot \lambda_{i} \int_{\theta_{i}^{R S}}^{+\infty} d F\left(\theta_{i}\right)+\left(S_{i}-c\right) \cdot \lambda_{-i} \int_{-\infty}^{\theta_{-i}^{R S}} d F\left(\theta_{-i}\right)
$$

Hence, the prices in the second period must satisfy the following by the first order approach:

$$
R_{i}^{m}=\frac{1-F\left(\theta_{i}^{R S}\right)}{f\left(\theta_{i}^{R S}\right)}+c \quad \text { and } \quad S_{i}^{m}=\frac{F\left(\theta_{-i}^{R S}\right)}{f\left(\theta_{-i}^{R S}\right)}+c .
$$

Note that there must exist unique solutions of $R_{i}^{m}$ and $S_{i}^{m}$ because the RHS of the two equations in (16) are decreasing in $R_{i}^{m}$ and $S_{i}^{m}$, respectively. 
We can find the cutoff types for any $\lambda_{i}$ :

$$
\theta_{i}^{R S}-\mu=R_{i}^{m}-S_{-i}^{m}=\frac{1-2 F\left(\theta_{i}^{R S}\right)}{f\left(\theta_{i}^{R S}\right)}
$$

hence,

$$
\theta_{i}^{R S}=\mu+\frac{1-2 F\left(\theta_{i}^{R S}\right)}{f\left(\theta_{i}^{R S}\right)}
$$

Suppose $\theta_{i}^{R S}<\mu$, then $\frac{1-2 F\left(\theta_{i}^{R S}\right)}{f\left(\theta_{i}^{R S}\right)}>0$ and hence the RHS of (17) must be greater than $\mu$, which is a contradiction. Suppose $\theta_{i}^{R S}>\mu$, then $\frac{1-2 F\left(\theta_{i}^{R S}\right)}{f\left(\theta_{i}^{R S}\right)}<0$ and hence the RHS of (17) must be less than $\mu$. This means that it must be true that $\theta_{i}^{R S}=\mu$, where $i \in\{A, B\}$. Plugging $\theta^{R S}=\mu$ back into the two equations for $R_{i}^{m}$ and $S_{i}^{m}$, respectively, we obtain the mass market equilibrium prices.

The above analysis is valid if and only if the prices indeed form a mass market equilibrium, i.e., $\theta^{R S}-R_{i}^{m}=\mu-\frac{1}{2 f(\mu)}-c>0$ is true. Hence, the sufficient and necessary condition for the existence of the above mass market equilibrium is

$$
\mu-\frac{1}{2 f(\mu)}-c>0
$$

Given that the function $x-[1-F(x)] / f(x)-c$ is monotonically increasing and that $M-[1-$ $F(M)] / f(M)-c=0$ holds, we have $\mu>M$.

Since the solutions to $R_{i}^{m}$ and $S_{i}^{m}$ are unique, and according to the first two parts of the proposition, there do not exist any niche or semi-niche market equilibria when $\mu>M$, the mass market equilibrium is the unique equilibrium.

Proof of Proposition 2. Note that $M>m$. This is true because $1-\rho \in(0,1)$ and hence, the RHS of (10) lies strictly below the RHS of (4).

Step 1. A unique niche market equilibrium where firm $i$ charges $M$ to repeat customers exists if and only if $\mu<\frac{c-\rho M}{1-\rho}$.

In such an equilibrium, the firm charges the monopoly price to repeat customers. Hence, there is no profitable deviation in terms of the price to repeat customers.

For there to be no profitable deviation of prices to switchers, it has to be true that no firm finds it profitable to charge a price no less than marginal cost to attract customers of the competitor who charges monopoly price. Thus, it has to be true that given firm $-i$ charges $M$ 
to its repeat customers, firm $i$ does not find it profitable to charge $S_{i} \geq c$ to attract some of firm $-i$ 's consumers to switch to product $i$. This means that the equilibrium exists if and only if the following condition is satisfied $\rho M+(1-\rho) \mu<c$, i.e., $\mu<\frac{c-\rho M}{1-\rho}$.

Regarding uniqueness, suppose there exists an alternative niche market equilibrium in which both firms charge a price $\tilde{P} \neq M$. According to the definition of niche market equilibrium, there are no switching consumers and firms do not face any competition in their own territory in the second period. This suggests that the optimal second-period price must be $M$. A contradiction. Thus, there does not exist an alternative niche market equilibrium and the proposition gives the unique niche market equilibrium.

Step 2. A semi-niche market equilibrium where firm $i$ charges $R_{i}^{s}$ to repeat customers and $\rho R_{i}^{s}+(1-\rho) \mu$ to switching ones, where $R_{i}^{s} \in[m, \iota]$ and $\iota \in[m, M]$, exists if and only if $\mu \leq \frac{c-\rho \iota}{1-\rho}$.

The equilibrium is a knife-edge case as the surplus curve of repeat customers and switching customers cross at the horizontal axis. To see why, note that

$$
\theta_{i}^{R S}=\mu+\frac{R_{i}^{s}-S_{-i}^{s}}{1-\rho}=\mu+\frac{R_{i}^{s}-\left[\rho R_{i}^{s}+(1-\rho) \mu\right]}{1-\rho}=R_{i}^{s}
$$

and thus, $\theta_{i}^{R S}-R_{i}^{s}=\rho \theta_{i}^{R S}+(1-\rho) \mu-\left[\rho R_{i}^{s}+(1-\rho) \mu\right]=0$. This means that $\theta_{i}^{R S}=\theta_{i}^{R O}=\theta_{i}^{S O}$.

To prove that the equilibrium exists for a given $R_{i}^{s} \in[m, \iota]$, first suppose firm $i$ deviates to $R_{i} \geq R_{i}^{s}$, then its profit, given firm $-i$ 's price $S_{-i}^{s}=\rho R_{i}^{s}+(1-\rho) \mu$, is given by

$$
\lambda_{i}\left(R_{i}-c\right) \int_{\theta_{i}^{R S}}^{\infty} d F(\theta)=\lambda_{i}\left(R_{i}-c\right) \int_{\mu+\frac{R_{i}-S_{-i}^{s}}{1-\rho}}^{\infty} d F(\theta)
$$

Taking the first order derivative w.r.t $R_{i}$, we have:

$$
\begin{aligned}
& \lambda_{i}\left[1-F\left(\theta_{i}^{R S}\right)\right]\left[1-\frac{R_{i}-c}{1-\rho} \frac{f\left(\theta_{i}^{R S}\right)}{1-F\left(\theta_{i}^{R S}\right)}\right] \\
= & \lambda_{i}\left[1-F\left(\frac{R_{i}-\rho R_{i}^{s}}{1-\rho}\right)\right]\left[1-\frac{R_{i}-c}{1-\rho} \frac{f\left(\frac{R_{i}-\rho R_{i}^{s}}{1-\rho}\right)}{1-F\left(\frac{R_{i}-\rho R_{i}^{s}}{1-\rho}\right)}\right] .
\end{aligned}
$$

Since $R_{i} \geq R_{i}^{s}$, then $\theta_{i}^{R S}=\frac{R_{i}-\rho R_{i}^{s}}{1-\rho} \geq R_{i}^{s}$ and thus, we have:

$$
\frac{R_{i}-c}{1-\rho} \frac{f\left(\frac{R_{i}-\rho R_{i}^{s}}{1-\rho}\right)}{1-F\left(\frac{R_{i}-\rho R_{i}^{s}}{1-\rho}\right)} \geq \frac{R_{i}^{s}-c}{1-\rho} \frac{f\left(R_{i}^{s}\right)}{1-F\left(R_{i}^{s}\right)} \geq \frac{m-c}{1-\rho} \frac{f(m)}{1-F(m)} \equiv 1,
$$

where the second inequality is due to $R_{i}^{s} \geq m$. This implies that the first order derivative (18) is negative if $R_{i}>R_{i}^{s}$ and firm $i$ does not find it profitable to deviate to a price above $R_{i}^{s}$. 
Suppose instead firm $i$ deviates to $R_{i}<R_{i}^{s}$, then the corresponding profit function is given by

$$
\lambda_{i}\left(R_{i}-c\right) \int_{R_{i}}^{\infty} d F(\theta)
$$

which takes its maximum at $M \geq R_{i}^{s}$. Hence, the first order derivative w.r.t $R_{i}$ is positive for $R_{i}<R_{i}^{s} \leq M$ and it is not a profitable deviation.

Firm -i's profit from switching customers in this equilibrium is zero, as there is effectively zero measure of customers who switch. Firm $-i$ does not find it profitable to deviate to a price greater than $c$ if and only if $\rho \theta_{i}^{R S}+(1-\rho) \mu=\rho R_{i}^{s}+(1-\rho) \mu \leq c$, i.e., $\mu \leq \frac{c-\rho R_{i}^{s}}{1-\rho}$. If $\mu \leq \frac{c-\rho \iota}{1-\rho}$, then $\mu \leq \frac{c-\rho R_{i}^{s}}{1-\rho}$ holds for any $R_{i}^{s} \in[m, \iota]$, hence, firm $-i$ does not wish to deviate. On the other hand, if a semi-niche market equilibrium exists for each $R_{i}^{s} \in[m, \iota]$, then it must be true that $\mu \leq \frac{c-\rho \iota}{1-\rho}$ because it implies that $\mu \leq \frac{c-\rho R_{i}^{s}}{1-\rho}$ holds for any $R_{i}^{s} \in[m, \iota]$.

Step 3. A unique equilibrium, which is a mass market equilibrium, exists if and only if $\mu>\frac{c-\rho m}{1-\rho}$

We now start with proving sufficiency, i.e., if $\mu>\frac{c-\rho m}{1-\rho}$ then the mass market equilibrium exists.

First, we show that $R_{i}^{m}>S_{i}^{m}>c$, assuming that the prices given by (8) and (9) are indeed an equilibrium. Since the equilibrium is symmetric, proving $R_{i}^{m}>S_{i}^{m}$ is equivalent to proving that $R_{i}^{m}>S_{-i}^{m}$. Suppose $R_{i}^{m} \leq S_{-i}^{m}$ instead, then it must be true that $\theta_{i}^{S O}<\theta_{i}^{R S} \leq \mu$ and, thus, that $S_{-i}^{m}$ must satisfy the following inequalities:

$S_{-i}^{m}-c=\frac{(1-\rho)\left[F\left(\theta_{i}^{R S}\right)-F\left(\theta_{i}^{S O}\right)\right]}{f\left(\theta_{i}^{R S}\right)+\frac{1-\rho}{\rho} f\left(\theta_{i}^{S O}\right)}<\frac{(1-\rho)\left[F\left(\theta_{i}^{R S}\right)-F\left(\theta_{i}^{S O}\right)\right]}{f\left(\theta_{i}^{R S}\right)}<\frac{(1-\rho)\left[1-F\left(\theta_{i}^{R S}\right)\right]}{f\left(\theta_{i}^{R S}\right)}=R_{i}^{m}-c$.

The last inequality is due to $1-F\left(\theta_{i}^{R S}\right) \geq \frac{1}{2}>F\left(\theta_{i}^{R S}\right)-F\left(\theta_{i}^{S O}\right)$ because of $\theta_{i}^{S O}<\theta_{i}^{R S} \leq \mu$. Hence, there is a contradiction. Given that $R_{i}^{m}>S_{-i}^{m}$, we have $\theta_{i}^{R S}>\mu$.

Second, we prove that (8) and (9) are indeed an equilibrium strategy, given that $\mu \geq \frac{c-\rho m}{1-\rho}$. The following first order derivative of (5) with $\theta^{R S}>\theta^{R O}>\theta^{S O}>-\infty$ w.r.t $R_{i}$ equals zero when replacing $R_{i}$ with (8):

$$
\lambda_{i}\left[1-F\left(\theta_{i}^{R S}\right)\right]\left[1-\frac{R_{i}-c}{1-\rho} \frac{f\left(\theta_{i}^{R S}\right)}{1-F\left(\theta_{i}^{R S}\right)}\right]=0
$$

To show that (8) is the maximizer and not a minimizer, recall our assumption on the monotone hazard rate condition and the fact that $\theta_{i}^{R S}$ increases in $R_{i}$ due to (1) and $\rho>0$. Then, the derivative (19) is positive when $R_{i}<R_{i}^{m}$ and negative when $R_{i}>R_{i}^{m}$. Finally, note that fixing $S_{-i}^{m}$, the RHS of (8) is a decreasing function of $R_{i}^{m}$, and the LHS is increasing in $R_{i}^{m}$. Hence, 
there exists a unique $R_{i}^{m}$ for each $S_{-i}^{m}$.

Similarly, the first order derivative of (5) with $\theta^{R S}>\theta^{R O}>\theta^{S O}>-\infty$ w.r.t $S_{i}$ equals zero when replacing $S_{i}$ with (9):

$$
\begin{aligned}
& \lambda_{-i}\left[F\left(\theta_{-i}^{R S}\right)-F\left(\theta_{-i}^{S O}\right)\right]\left[1-\frac{S_{i}^{m}-c}{\rho(1-\rho)} \frac{\rho f\left(\theta_{-i}^{R S}\right)+(1-\rho) f\left(\theta_{-i}^{S O}\right)}{F\left(\theta_{-i}^{R S}\right)-F\left(\theta_{-i}^{S O}\right)}\right] \\
& =\lambda_{-i}\left[F\left(\theta_{-i}^{R S}\right)-F\left(\theta_{-i}^{S O}\right)\right][1-\frac{S_{i}^{m}-c}{\rho(1-\rho)} \underbrace{\frac{\rho \frac{f\left(\theta_{-i}^{R S}\right)}{1-F\left(\theta_{-i}^{S O}\right)}+(1-\rho) \frac{f\left(\theta_{-i}^{S O}\right)}{1-F\left(\theta_{-i}^{S O}\right)}}{\frac{F\left(\theta_{-i}^{R S}\right)-F\left(\theta_{-i}^{S O}\right)}{1-F\left(\theta_{-i}^{S O}\right)}}}_{h\left(S_{i}^{m}\right)}] \\
& =0 \text {. }
\end{aligned}
$$

In order for $S_{-i}^{m}$ given by (8) to be the profit maximizer, $h(\cdot)$ has to be an increasing function. We now show that the function $h(\cdot)$ is an increasing function. Suppose $\theta_{-i}^{S O}>\mu$, then $h(\cdot)$ is an increasing function because: (a) $\theta_{-i}^{S O}$ is increasing in $S_{i}$ while $\theta_{-i}^{R S}$ is decreasing in $S_{i}$, hence, the denominator of $h(\cdot)$ is decreasing in $S_{i}$; (b) $\frac{f(\cdot)}{1-F(\cdot)}$ is an increasing function; (c) $\frac{F\left(\theta_{-i}^{R S}\right)-F\left(\theta_{-i}^{S O}\right)}{1-F\left(\theta_{-i}^{S O}\right)}$ is decreasing in $S_{i}^{m}$, which can be shown by taking first order derivative w.r.t $S_{i}^{m}$. Hence, $h(\cdot)$ is increasing in $S_{i}$.

Suppose instead $\theta_{i}^{S O} \leq \mu$, then $f^{\prime}\left(\theta_{-i}^{S O}\right) \geq 0$. The first order derivative w.r.t $S_{i}$ of $h\left(S_{i}\right)$ is:

$$
\begin{aligned}
\frac{d h\left(S_{i}\right)}{d S_{i}}= & \frac{1}{\left[F\left(\theta_{-i}^{R S}\right)-F\left(\theta_{-i}^{S O}\right)\right]^{2}}\left\{\left[\rho f^{\prime}\left(\theta_{-i}^{R S}\right) \frac{d \theta_{-i}^{R S}}{d S_{i}}+(1-\rho) f^{\prime}\left(\theta_{-i}^{S O}\right) \frac{d \theta_{-i}^{S O}}{d S_{i}}\right]\left[F\left(\theta_{-i}^{R S}\right)-F\left(\theta_{-i}^{S O}\right)\right]\right. \\
& \left.-\left[\rho f\left(\theta_{-i}^{R S}\right)+(1-\rho) f\left(\theta_{-i}^{S O}\right)\right]\left[f\left(\theta_{-i}^{R S}\right) \frac{d \theta_{-i}^{R S}}{d S_{i}}-f\left(\theta_{-i}^{S O}\right) \frac{d \theta_{-i}^{S O}}{d S_{i}}\right]\right\} \\
= & \frac{1}{\left[F\left(\theta_{-i}^{R S}\right)-F\left(\theta_{-i}^{S O}\right)\right]^{2}}\left\{\left[-f^{\prime}\left(\theta_{-i}^{R S}\right) \frac{\rho}{1-\rho}+f^{\prime}\left(\theta_{-i}^{S O}\right) \frac{1-\rho}{\rho}\right]\left[F\left(\theta_{-i}^{R S}\right)-F\left(\theta_{-i}^{S O}\right)\right]\right. \\
& \left.+\left[\rho f\left(\theta_{-i}^{R S}\right)+(1-\rho) f\left(\theta_{-i}^{S O}\right)\right]\left[f\left(\theta_{-i}^{R S}\right) \frac{1}{1-\rho}+f\left(\theta_{-i}^{S O}\right) \frac{1}{\rho}\right]\right\}>0
\end{aligned}
$$

where $\frac{d \theta_{-i}^{R S}}{d S_{i}}=-\frac{1}{1-\rho}$ and $\frac{d \theta_{-i}^{S O}}{d S_{i}}=\frac{1}{\rho}$. The above derivative is positive because $f^{\prime}\left(\theta_{-i}^{R S}\right)<0$, due to $\theta_{-i}^{R S}>\mu$, and $f^{\prime}\left(\theta_{-i}^{S O}\right) \geq 0$. Thus, again we know that $h(\cdot)$ is an increasing function and $S_{-i}^{m}$ is the unique maximizer of (5) given each $R_{i}^{m}$.

Last, we prove that $\theta_{i}^{R S}-R_{i}^{m}>0$ when $\mu>\frac{c-\rho M}{1-\rho}$, that is, the marginal repeat consumer strictly prefers to stay in the market rather than choosing the outside option. Suppose instead $\theta_{i}^{R S}-R_{i}^{m} \leq 0$, then it must be true that $R_{i}^{m} \geq m$. To see why, suppose $m>R_{i}^{m} \geq \theta_{i}^{R S}$. Then

$$
m=\frac{(1-\rho)[1-F(m)]}{f(m)}+c<\frac{(1-\rho)\left[1-F\left(\theta_{i}^{R S}\right)\right]}{f\left(\theta_{i}^{R S}\right)}+c=R_{i}^{m}
$$


where the inequality is due to monotone hazard rate assumption. A contradiction. Thus, $R_{i}^{m} \geq m$. Since $\theta_{i}^{R S}-R_{i}^{m} \leq 0$ implies $\rho \theta_{i}^{R S}+(1-\rho) \mu \leq S_{-i}^{m}$, which in turn implies $\theta_{i}^{S O} \geq \theta_{i}^{R S}$ according to $S_{-i}^{m} \equiv \rho \theta_{i}^{S O}+(1-\rho) \mu$, thus $S_{-i}^{m} \leq c$ due to (9).

Now we show that if $\theta_{i}^{R S}-R_{i}^{m} \leq 0$, then $\rho R_{i}^{m}+(1-\rho) \mu \leq c$. Suppose the reverse is true, then $\rho R_{i}^{m}+(1-\rho) \mu>c \geq S_{-i}^{m}$, which implies $\left[\rho R_{i}^{m}+(1-\rho) \mu\right]-\left[\rho \theta_{i}^{S O}+(1-\rho) \mu\right]=\rho\left(\theta_{i}^{R O}-\theta_{i}^{S O}\right)>0$ and hence $\theta_{i}^{R O}>\theta_{i}^{S O}$. Thus, we have $\theta_{i}^{R O}>\theta_{i}^{S O} \geq \theta_{i}^{R S}$. When $\theta_{i}^{R S}=\theta_{i}^{S O}$, we have $\rho \theta_{i}^{R S}+(1-\rho) \mu-S_{i}^{m}=0$, which then implies $\theta_{i}^{R S}-\theta_{i}^{R O}=\theta_{i}^{R S}-R_{i}^{m}=0$, which contradicts $\theta_{i}^{R S}<\theta_{i}^{R O}$. When $\theta_{i}^{R S}<\theta_{i}^{S O}$ and hence $\theta_{i}^{R S}<\theta_{i}^{S O}<\theta_{i}^{R O}$, then we have $\rho=\frac{\theta_{i}^{R S}-\theta_{i}^{R O}}{\theta_{i}^{R S}-\theta_{i}^{S O}}>1$, which contradicts $\rho \in(0,1)$. Hence, $\rho R_{i}^{m}+(1-\rho) \mu \leq c$.

In sum, if $\theta_{i}^{R S}-R^{m} \leq 0$ is true, then $\rho R_{i}^{m}+(1-\rho) \mu \leq c$ which implies $\rho m+(1-\rho) \mu \leq c$, i.e., $\mu \leq \frac{c-\rho m}{1-\rho}$. A contradiction. Therefore, it must be true that $\theta_{i}^{R S}-R_{i}^{m}>0$.

Now we prove necessity, i.e., if there exists a mass market equilibrium, then $\mu>\frac{c-\rho m}{1-\rho}$. Suppose $m \leq R_{i}^{m}$, then according to $\theta_{i}^{R S}>R_{i}^{m}$ in the mass market equilibrium, we have

$$
m=\frac{(1-\rho)[1-F(m)]}{f(m)}+c>\frac{(1-\rho)\left[1-F\left(\theta_{i}^{R S}\right)\right]}{f\left(\theta_{i}^{R S}\right)}+c=R_{i}^{m} .
$$

A contradiction. Hence, $m>R_{i}^{m}$. Suppose $\mu \leq \frac{c-\rho m}{1-\rho}$, then $\rho R_{i}^{m}+(1-\rho) \mu<\rho m+(1-\rho) \mu \leq c$. This, and the fact that in the mass market equilibrium with positive correlation $\theta_{i}^{R S}>\theta_{i}^{R O}>$ $\theta_{i}^{S O}$ holds, imply that $S_{i}^{m} \equiv \rho \theta_{i}^{S O}+(1-\rho) \mu<\rho \theta_{i}^{R O}+(1-\rho) \mu \equiv \rho R_{i}^{m}+(1-\rho) \mu<c<S_{i}^{m}$. A contradiction. Hence, it must be true that $\mu>\frac{c-\rho m}{1-\rho}$.

Regarding uniqueness, since the solutions to $R_{i}^{m}$ and $S_{i}^{m}$ given by (8) and (9) are unique, we know that the mass market equilibrium must be the unique mass market equilibrium. No niche and semi-niche market equilibria exist either due to $\mu>\frac{c-\rho m}{1-\rho}$, according to step 1 and 2 of this proof.

Proof of Proposition 3. According to Proposition 1, each firm makes monopoly profit from their repeat customers but no profit from switching customers as no one switches in the second period if and only if $\mu \leq c$. This suggests firms would compete for first-period market share by lowering the first-period price until the gain in the second period from additional market share balances the loss from capturing firm $-i$ 's market share in the first period, i.e.,

$$
\underbrace{\lambda_{-i}\left(c-p_{i}^{n}\right)}_{\text {loss }}=\underbrace{\delta \lambda_{-i} \pi^{M}}_{\text {gain }}
$$

Neither firm prefers to deviate from such an equilibrium. If firm $i$ deviates to any price higher 
than $p_{i}^{n}$ in the first period, its first period profit increases by $\lambda_{i}\left(c-p_{i}^{n}\right)$ to 0 and its second period profit decreases by $\lambda_{i} \pi(M)$, thus the total change of its profit is 0 since $\lambda_{i}\left(c-p_{i}^{n}\right)-\delta \lambda_{i} \pi(M)=0$. If, instead, firm $i$ deviates to a lower price $p_{i}^{n}-\epsilon$ where $\epsilon$ is arbitrarily small in the first period, then its first period profit decreases by $\lambda_{-i}\left(c-p_{i}^{n}\right)$ and its second period profit increases by $\lambda_{-i} \pi(M)$, and the total change of profit is also 0. Any price even lower than $p_{i}^{n}-\epsilon$ is strictly dominated. Hence, there is no profitable deviation for firms.

Note that the above proof applies to any $\lambda_{i} \in[0,1]$. This implies that when firm $i$ sells only in the second period while firm $-i$ sells in both periods such that $\lambda_{i}=0$, both firms' profit stays unchanged. When firm $i$ sells only in the second period given that firm $-i$ does the same, demand is zero because no consumer would purchase as $\mu-c<0$. Hence, they also make zero profit. This means that there exists a variety of zero-profit equilibria in which either $\lambda_{i}+\lambda_{-i}=1$ when $\max \left(\lambda_{i}, \lambda_{-i}\right)>0$ or $\max \left(\lambda_{i}, \lambda_{-i}\right)=0$.

Consumers' surplus is given by

$$
\begin{aligned}
& (\mu-c)+\delta \pi(M)+\delta \int_{M}^{\infty}(x-M) d F(x) \\
= & (\mu-c)+\delta \pi(M)+\delta \int_{M}^{\infty}(x-c) d F(x)-\delta \int_{M}^{\infty}(M-c) d F(x) \\
= & (\mu-c)+\delta \int_{M}^{\infty}(x-c) d F(x) .
\end{aligned}
$$

Hence, the consumer surplus is only non-negative if and only if the total discounted social surplus over the two periods is non-negative. Since purchasing only in the second period yields a payoff of $\mu-S_{i}^{n}<c-S_{i}^{n} \leq 0$, all consumers wish to buy in the first period.

Proof of Proposition 4. Note that according to Proposition 1, all the second period equilibrium prices are invariant to $\lambda_{i}$. Hence, consumers' expected surplus from the second period is invariant to their purchasing decision in the first period, which implies that they would purchase the good with the lower price in the first period.

We now prove the proposition in two steps.

\section{Step 1. The equilibrium in the second-period sub-game is the mass market equilibrium}

In the mass market, each firm makes exactly the same profit from the repeat and the switching customers and thus, its second period profit does not vary with the first period market share. Hence, firms compete in the first period as in Bertrand competition by lowering their first period prices to the marginal cost. 
Consumers' discounted surplus over the two periods is given by

$$
(\mu-c)+\delta[\underbrace{\int_{\theta_{i}^{R S}}^{\infty}\left(x-R_{i}^{m}\right) d F(x)+\int_{-\infty}^{\theta_{i}^{R S}}\left[E\left(\theta_{-i} \mid x\right)-S_{-i}^{m}\right] d F(x)}_{\geq 0}]>0 .
$$

Consumers must have non-negative surplus in the second period since they are free to leave the market otherwise, hence the terms in the brackets must be non-negative. The above consumer surplus is positive since $\mu \geq M>c$. It is also easy to verify that the above consumer surplus is no less than the surplus from purchasing only in the second period, given by $\delta\left(\mu-S_{i}^{m}\right)=$ $\delta\left(\mu-\frac{1}{2 f(\mu)}-c\right)$. Thus, consumers are willing to purchase one of the products in the first period.

Firms do not wish to sell only in the second period for the following reasons. Given that firm $-i$ sells in both periods, selling only in the second period yields firm $i$ the same profit as given in the Proposition. If firm $-i$ were selling only in the second period, firm $i$ would find it not profitable to sell only in the second period because Bertrand competition would follow and both firms would make zero profit. Therefore, selling only in the second period is weakly dominated for each firm.

Step 2. The equilibrium in the second-period sub-game is the semi-niche market equilibrium Suppose $\pi(M)=(M-c)[1-F(M)] \geq(\mu-c) F(M)$, then firms compete for higher market share in the first period by lowering price. Then through a procedure similar to Step 1 we can prove that the first period equilibrium price must satisfy $p_{i}^{s}=c-\delta[\pi(M)-(\mu-c) F(M)]$.

For consumers, their surplus is given by

$$
(\mu-c)+\delta[\pi(M)-(\mu-c) F(M)]+\delta \int_{M}^{\infty}(x-M) d F(x)>0
$$

which is positive since $\mu \geq c, \pi(M) \geq(\mu-c) F(M)$, and $\int_{M}^{\infty}(x-M) d F(x)>0$. Since purchasing only in the second period also yields $\delta(\mu-\mu)=0$, the fact that the above consumer surplus is positive implies that no consumer wishes to purchase only in the first period. Hence, all consumers are willing to purchase one of the products in the first period.

Suppose instead, $\pi(M)<(\mu-c) F(M)$, i.e., $(M-c)[1-F(M)]<(\mu-c) F(M)$, then firms may prefer less market share in the first period as they make greater profit from poaching customers from the competitor. Then, they would increase price in the first period up to a point

$$
\lambda_{i}\left(p_{i}^{s}-c\right)=\delta \lambda_{i}[(\mu-c) F(M)-\pi(M)]
$$


where the LHS is the profit of firm $i$ by obtaining a market share in the first period and the RHS is the opportunity cost of getting $\lambda_{i}$ of first-period market share. Suppose firm $i$ deviates to an even higher price, then it loses the profit $\lambda_{i}\left(p_{i}^{s}-c\right)$ in the first period and obtains a gain of $\delta \lambda_{i}[(\mu-c) F(M)-\pi(M)]$ in the second period. The loss and the gain balance each other, which implies that the deviation is not profitable. Alternatively, suppose firm $i$ deviates to a slightly lower price, then it obtains an additional profit of $\lambda_{-i}\left(p_{i}^{s}-c\right)$ in the first period and loses the profit of $\delta \lambda_{-i}[(\mu-c) F(M)-\pi(M)]$ in the second period. Again, the two balance each other and the deviation is not profitable. Any price lower than $p_{i}^{s}-\epsilon$, where $\epsilon$ is sufficiently small, is dominated. Hence, the firm charges exactly the same first-period price as when $\pi(M) \geq(\mu-c) F(M)$.

Note that, as in the proof of Proposition 3, firms' equilibrium profit is a constant for any $\lambda_{i} \in[0,1]$ such that $\lambda_{i}+\lambda_{-i}=1$. Furthermore, firms end up in Bertrand competition and make zero profit if both of them sell only in the second period. Therefore, selling only in the second period is weakly dominated.

For consumers, the surplus is given by

$$
\begin{aligned}
& (\mu-c)-\delta((\mu-c) F(M)-\pi(M))+\delta \int_{M}^{\infty}(x-M) d F(x) \\
= & (\mu-c)-\delta((\mu-c) F(M)-\pi(M))+\delta \int_{M}^{\infty}(x-c) d F(x)-\delta \int_{M}^{\infty}(M-c) d F(x) \\
= & (\mu-c)-\delta(\mu-c) F(M)+\delta \int_{M}^{\infty}(x-c) d F(x) \\
= & (\mu-c)[1-\delta F(M)]+\delta \int_{M}^{\infty}(x-c) d F(x)>0 .
\end{aligned}
$$

The above consumer surplus is positive because $\mu \geq c$ and $M>c$. This implies that no consumer wishes to purchase only in the second period. Hence, consumers are willing to purchase one of the products in the first period.

Proof of Proposition 5. Recall from Proposition 2 in the paper that there are no switchers in either the niche or the semi-niche market equilibrium. We can hence prove the first period prices given in the proposition following the same procedure as for Proposition 3.

Suppose firm $i$ deviates to a price slightly lower than the $p_{i}^{k}$ where $k \in\{n, s\}$ given in the proposition, then it obtains all the market in the first period, i.e., $\lambda_{i}=1$. Its second period profit increases by $\delta \lambda_{-i} \Pi^{k}$ (where $\Pi^{n}=\pi(M)$ and $\Pi^{s}=\pi\left(R_{i}^{s}\right)$ ) while its first period profit decreases by $\lambda_{-i}\left(c-p_{i}^{k}\right)=\lambda_{-i} \delta \Pi^{k}$, which is the same as the former profit increment. Hence, any price lower than $p_{i}^{k}$ is not a profitable deviation. Alternatively, suppose firm $i$ deviates to a 
price higher than $p_{i}^{k}$, then it loses all market share in the first period. Given the second period equilibrium prices, no consumers would switch from the competitor to firm $i$, hence, firm $i$ 's total profit is zero. Thus, raising first period price above $p_{i}^{k}$ is not a profitable deviation.

Note again that the proof here applies to any $\lambda_{i} \in[0,1]$ such that $\lambda_{i}+\lambda_{-i}=1$ and that when both firms sell only in the second period they end up in Bertrand competition with zero profits, which is no greater than their equilibrium profit of zero. Therefore, there is no profitable deviation for either firm in terms of first period price and whether to sell only in the second period.

Regarding the consumers' choice, we start by the first period choice given that firms play the niche market equilibrium in the second period, i.e., given $\mu \leq \frac{c-\rho M}{1-\rho}$. Consumers' surplus given that $\mu \leq \frac{c-\rho M}{1-\rho}$ is

$$
\begin{aligned}
& \left(\mu-p_{i}^{n}\right)+\delta \int_{M}^{\infty}(x-M) d F(x) \\
= & (\mu-c)+\delta \pi(M)+\delta \int_{M}^{\infty}(x-c) d F(x)-\delta \int_{M}^{\infty}(M-c) d F(x) \\
= & (\mu-c)+\delta \underbrace{\int_{M}^{\infty}(x-c) d F(x)}_{S S(M)}
\end{aligned}
$$

Hence, the above consumer surplus is non-negative if and only if $\mu-c \geq-\delta \cdot S S(M)$. Since purchasing only in the second period yields a payoff of $\mu-S_{i}^{n}<\frac{c-\rho \cdot M}{1-\rho}-S_{i}^{n}<c-S_{i}^{n} \leq 0$, no consumer prefers to do so.

Next, we turn to the first period choice of consumers followed by a semi-niche market equilibrium in the second period. Recall from Lemma 2 in the paper, a continuum of semi-niche market equilibria exists if and only if $\mu \leq \frac{c-\rho \iota}{1-\rho}$ with $\iota \in[m, M]$. There are no switchers in the semi-niche market equilibrium given that $\rho>0$.

Consumers' surplus given a specific semi-niche market with prices $R_{i}^{s} \in[m, \iota]$ and $S_{i}^{s}=$ $\rho R_{i}^{s}+(1-\rho) \mu$ and $\mu \leq \frac{c-\rho R_{i}^{s}}{1-\rho}$ is the following:

$$
\begin{aligned}
& \left(\mu-p_{i}^{s}\right)+\delta \int_{R_{i}^{s}}^{\infty}\left(x-R_{i}^{s}\right) d F(x) \\
= & (\mu-c)+\delta \cdot \pi\left(R_{i}^{s}\right)+\delta \int_{R_{i}^{s}}^{\infty}(x-c) d F(x)-\delta \int_{R_{i}^{s}}^{\infty}\left(R_{i}^{s}-c\right) d F(x) \\
= & (\mu-c)+\delta \underbrace{\int_{R_{i}^{s}}^{\infty}(x-c) d F(x)}_{S S\left(R_{i}^{s}\right)} .
\end{aligned}
$$

Hence, the above consumers' surplus is non-negative if and only if $\mu-c \geq-\delta \cdot S S\left(R_{i}^{s}\right)$. Suppose 
a consumer purchased only in the second period, then her surplus is given by $\mu-S_{i}^{s}=\mu-$ $\rho R_{i}^{s}-(1-\rho) \mu=\rho\left(\mu-R_{i}^{s}\right)<\rho(c-m)<0$. Hence, all consumers purchase one of the products in the first period.

Proof of Proposition 6. According to Proposition 2, the second period equilibrium prices given any $\lambda_{i}$ are invariant of $\lambda_{i}$. Hence, consumers' expected surplus from the second period is invariant to their purchasing decision in the first period. Thus, consumers purchase the good with the lowest price in the first period.

In the first period, firms compete by choosing a price such that:

$$
\lambda_{-i}\left(c-p_{i}^{m}\right)=\delta\left(\lambda_{-i} \pi_{i}^{R m}-\lambda_{-i} \pi_{i}^{S m}\right) .
$$

Suppose the profit from repeat consumers is greater than from switchers, then firms compete by lowering their prices to the point satisfying (20). In this way, the first period loss equals the second period gain. Neither firm prefers to deviate from such an equilibrium. If firm $i$ deviates to any price higher than $p_{i}^{m}$ in the first period, its first period profit increases by $\lambda_{i}\left(c-p_{i}^{m}\right)$ to 0 and its second period profit decreases by $\lambda_{i} \pi_{i}^{R m}-\lambda_{i} \pi_{i}^{S m}$, thus the total change of its profit is 0 since, according to $(20), \lambda_{i}\left(c-p_{i}^{m}\right)-\delta\left(\lambda_{i} \pi_{i}^{R m}-\lambda_{i} \pi_{i}^{S m}\right)=0$. If, instead, firm $i$ deviates to a slightly lower price $p_{i}^{m}-\epsilon$ where $\epsilon$ is arbitrarily small, then its first period profit decreases by $\lambda_{-i}\left(c-p_{i}^{m}\right)$ and its second period profit increases by $\lambda_{-i} \pi_{i}^{R m}-\lambda_{-i} \pi_{i}^{S m}$, and the total change of profit is also 0 . Any price even lower than $p_{i}^{m}-\epsilon$ is strictly dominated. Hence, there is no profitable deviation. Suppose instead that firms make a greater profit from switcher than from repeat consumers, then they would increase their price in the first period up to a point where (20) is satisfied. A similar argument that there is no profitable deviation follows.

For consumers, their surplus given the equilibrium prices under the condition $\mu \geq \frac{c-\rho m}{1-\rho}$ is:

$$
\begin{aligned}
& \left(\mu-p_{i}^{m}\right)+\delta\left[\int_{\theta_{i}^{R S}}^{\infty}\left(x-R_{i}^{m}\right) d F(x)+\int_{\theta_{i}^{S O}}^{\theta_{i}^{R S}}\left[E\left(\theta_{-i} \mid x\right)-S_{-i}^{m}\right] d F(x)\right] \\
= & (\mu-c)+\delta\left[\int_{\theta_{i}^{R S}}^{\infty}\left(x-R_{i}^{m}\right) d F(x)+\pi_{i}^{R m}-\pi_{i}^{S m}+\int_{\theta_{i}^{S O}}^{\theta_{i}^{R S}}\left[E\left(\theta_{-i} \mid x\right)-S_{-i}^{m}\right] d F(x)\right] \\
= & (\mu-c)+\delta[\underbrace{\int_{\theta_{i}^{R S}}^{\infty}(x-c) d F(x)+\int_{\theta_{i}^{S O}}^{\theta_{i}^{R S}}\left[E\left(\theta_{-i} \mid x\right)-c\right] d F(x)}_{S S^{m}}-2 \cdot \pi_{i}^{S m}] .
\end{aligned}
$$

In equilibrium, the above consumers' surplus must be no less than the maximum of zero and 
the consumer surplus from purchasing only in the second period, i.e., $\delta\left(\mu-S_{i}^{m}\right)$. Hence, the sufficient and necessary condition for the consumer to be willing to follow the equilibrium and purchase one of the product is given by:

$$
\mu-c \geq-\delta \cdot\left(S S^{m}-2 \cdot \pi_{i}^{S m}\right)+\max \left[0, \delta\left(\mu-S_{i}^{m}\right)\right]
$$

where $S S^{m}=\int_{\theta_{i}^{R S}}^{\infty}(x-c) d F(x)+\int_{\theta_{i}^{S O}}^{\theta_{i}^{R S}}\left[E\left(\theta_{-i} \mid x\right)-c\right] d F(x)$.

\section{Negative information spillovers, $\rho \in[-1,0)$}

When $\rho$ is negative, information spillovers between new goods allow consumers to learn from a bad experience that they are likely to have a high valuation for the untried product. Since our interest is in competition between similar products and an outside option, this case is less relevant. Nonetheless, it is still useful to understand the equilibrium behavior in the negative correlation setting because the case of no information spillover setting $(\rho=0)$ analyzed in Section 3 is a special case of the equilibrium with $\rho \in[-1,0)$.

Theorem 1 in the paper is shown to also apply over this range of $\rho$, and some interesting contrasts emerge in comparing equilibria. Whereas when $\rho \in(0,1)$, the mass market led to price discrimination in the second period, and created the possibility of first-period profits, the mass market equilibrium when $\rho \in[-1,0]$ generalizes the findings for $\rho=0$ that the prices to repeat and switching consumers are equal, the market is split equally between these consumer segments, profits are equal for each segment, and first-period price is always equal to marginal cost. That is, there is Bertrand competition in the first period, and no dynamic pricing interdependencies, whenever there is a mass market equilibrium.

It is in the niche and semi-niche equilibria when $\rho \in[-1,0]$ that firms price discriminate and make profit from switching consumers. The relative profitability of repeat and switching consumers determines the intensity of first-period price competition in ways that are similar to the mass market equilibrium outcomes for $\rho \in(0,1)$.

We use backward induction to characterize the sub-game perfect equilibrium of the game for this case as well, and start by characterizing the second-period equilibria.

\section{C.1 Second-period equilibria}

In the second period, there are again three forms of equilibria: a niche market equilibrium, illustrated in Figure C.2, where some customers leave the market; a mass market equilibrium, illustrated in Figure C.4, where no customers leave the market; and a semi-niche equilibrium, 
illustrated in Figure C.3, where the marginal repeat consumer is indifferent between repeat buying, switching, or leaving the market. In each of these figures, observe that the expected

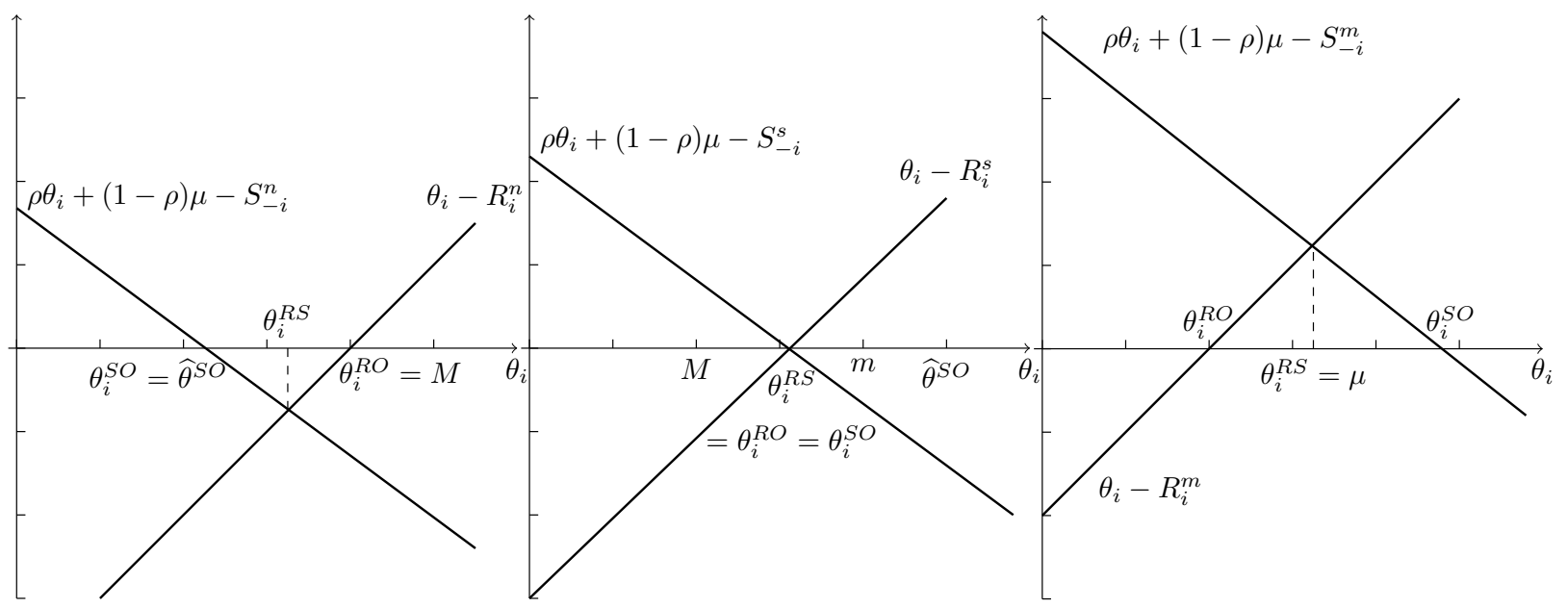

Figure C.2: Niche market equilibrium, where $R_{i}^{n}=M$ and $S_{-i}^{n}=\widehat{S}$, given by $(23)$.
Figure C.3: Semi-niche market equilibrium, where $R_{i}^{s}, S_{-i}^{s}$ satisfies (24) and (25)
Figure C.4: Mass market equilibrium, where $R_{i}^{m}=$ $S_{-i}^{m}=(1-\rho) /[2 f(\mu)]+c$.

value of consuming $-i$ in the second period is negatively sloped. Note that the new market is fully covered in the mass and semi-niche market equilibria when $\rho \in[-1,0]$ in contrast to these equilibria when $\rho \in(0,1)$.

Accordingly, firm $i$ 's second-period profit can be written as:

$$
\underbrace{\left(R_{i}-c\right) \lambda_{i} \int_{\max \left(\theta_{i}^{R S}, \theta_{i}^{R O}\right)}^{+\infty} d F\left(\theta_{i}\right)}_{\text {profit from repeat customers }}+\underbrace{\left(S_{i}-c\right) \lambda_{-i} \int_{-\infty}^{\min \left(\theta_{-i}^{R S}, \theta_{-i}^{S O}\right)} d F\left(\theta_{-i}\right)}_{\text {profit from switching customers }} .
$$

In a niche market equilibrium, we have $\max \left(\theta_{i}^{R S}, \theta_{i}^{R O}\right)=\theta_{i}^{R O}$ and $\min \left(\theta_{-i}^{R S}, \theta_{-i}^{S O}\right)=\theta_{-i}^{S O}$ in (21). In a semi-niche market equilibrium, $\theta_{i}^{R O}=\theta_{i}^{R S}=\theta_{i}^{S O}$ in (21). In a mass market equilibrium, we have $\max \left(\theta_{i}^{R S}, \theta_{i}^{R O}\right)=\theta_{i}^{R S}$ and $\min \left(\theta_{-i}^{R S}, \theta_{-i}^{S O}\right)=\theta_{-i}^{R S}$ in (21).

The prices in the niche market equilibrium can be characterized by the first order approach:

$$
\begin{aligned}
R_{i}^{n} & =M \equiv \frac{1-F(M)}{f(M)}+c, \\
S_{i}^{n} & =\widehat{S} \equiv-\frac{\rho F\left(\widehat{\theta}^{S O}\right)}{f\left(\widehat{\theta}^{S O}\right)}+c .
\end{aligned}
$$

where $\widehat{\theta}^{S O}=\frac{\widehat{S}-(1-\rho) \mu}{\rho}$. Note that we drop the subscript in $(23)$ as the price and the cutoff type are independent of $i$ and that $\widehat{S}>c$ as $\rho<0$. The equilibrium prices in the mass market equilibrium can also be characterized by first order conditions with respect to prices. The 
semi-niche market equilibria are characterized by construction.

Lemma 1. Suppose $\rho \in[-1,0)$. Then in the second period:

- if and only if $M \geq \widehat{\theta}^{S O}$, there exists a niche market equilibrium with prices given by (22) and (23).

- if and only if $m \geq \mu$ and $M \leq \widehat{\theta}^{S O}$, there exists a continuum of semi-niche market equilibria with prices satisfying

$$
S_{-i}^{s}=\rho R_{i}^{s}+(1-\rho) \mu
$$

and

$$
\max (M, \mu) \leq R_{i}^{s} \leq \min \left(m, \widehat{\theta}^{S O}\right)
$$

- if and only if $m \leq \mu$, there exists a mass market equilibrium with prices

$$
R_{i}^{m}=S_{i}^{m}=\frac{1-\rho}{2 f(\mu)}+c
$$

In the niche market equilibrium characterized in Lemma 1, firms charge the monopoly price $M$ to their repeat customers and make the monopoly profit $\pi(M)$. This is the same as in the $\rho=0$ setting.

Corollary 7. Suppose $\rho \in[-1,0)$. In the second period,

- in the niche market equilibrium, the prices satisfy $R_{i}^{n}=M \geq S_{i}^{n}=\widehat{S}$, where the equality is true only when $\rho=-1$;

- in the semi-niche market equilibrium, the prices satisfy $R_{i}^{s} \geq S_{i}^{s}$.

Lemma 1 also shows that in the mass market equilibrium, as when $\rho=0$, firms charge the same price to repeat and switching customers despite having the option to price discriminate based on purchasing history. By equation (1) in the paper, this gives $\theta_{i}^{R S}=\mu$. These observations are perfectly consistent with the mass market equilibrium with $\rho=0$. In fact, the equilibrium prices given in Lemma 1 and the second-period profit of a firm in the mass market equilibrium, $\frac{1-\rho}{4 f(\mu)}$, are equal to the corresponding terms in Lemma 1 in the paper by setting $\rho=0$. 


\section{C.2 Sub-game perfect equilibrium of the two-stage pricing game}

Consumers purchase good $i$ in the first period if and only if the relevant sum of the surplus in the first period and the anticipated surplus in the second period is greater than the sum of surpluses from choosing $-i$ and zero. The consumer surplus from purchasing good $i$ in the first period is thus given by:

$$
\begin{aligned}
\underbrace{\left(\mu-p_{i}\right)}_{\text {consumer surplus from trying } i} & +\delta \underbrace{\int_{\max \left(\theta_{i}^{R O}, \theta_{i}^{R S}\right)}^{\infty}\left(x-R_{i}^{*}\right) d F(x)}_{\text {consumer surplus from sticking with } i} \\
& +\delta \underbrace{\int_{-\infty}^{\min \left(\theta_{i}^{S O}, \theta_{i}^{R S}\right)}\left[E\left(\theta_{-i} \mid x\right)-S_{-i}^{*}\right] d F(x)}_{\text {consumer surplus from switching to }-i \text { or to the outside option }}
\end{aligned}
$$

where $R_{i}^{*} \in\left\{R_{i}^{m}, R_{i}^{s}, R_{i}^{n}\right\}$, and $S_{i}^{*} \in\left\{S_{i}^{m}, S_{i}^{s}, S_{i}^{n}\right\}$.

Since, according to Lemma 1, firms make the same second-period profit from repeat and switching customers in the mass market equilibrium, they compete in the first period as in Bertrand competition. Hence, their first-period prices are equal to the marginal cost. This observation is similar to the positive correlation setting with the mass market equilibrium in the second period.

However, firms also make positive profits in the semi-niche and the niche market equilibra in the second period when consumer values are negatively correlated, whereas they make zero profit in these equilibria when values are positively correlated. This is again due to the observation that firms' market power arises from the ability to poach the competitor's customers and the fact that, according to Lemma 1, firms always make a positive profit from switching customers in the second period when the correlation is negative.

Proposition 7. When $\rho<0$ :

- if and only if $M \geq \widehat{\theta}^{S O}$ and $\mu \in\left[c-\delta\left(S S^{n}-2 \pi_{i}^{S n}\right)+\max (0, \delta(\mu-\widehat{S})), \infty\right)$, there exists an equilibrium where firm $i$ makes a profit of $\delta \pi_{i}^{S n}>0$ by charging $p_{i}^{n}=c-\delta\left(\pi(M)-\pi_{i}^{S n}\right)$ in the first period and $R_{i}^{n}, S_{i}^{n}$ as given by (22) and (23) in the second-period niche market equilibrium, where

$$
\begin{aligned}
S S^{n} & =\int_{M}^{\infty}(x-c) d F(x)+\int_{-\infty}^{\widehat{\theta}^{S O}}\left[E\left(\theta_{-i} \mid x\right)-c\right] d F(x), \\
\pi_{i}^{S n} & =-\frac{\rho F\left(\widehat{\theta}^{S O}\right)^{2}}{f\left(\widehat{\theta}^{S O}\right)} .
\end{aligned}
$$


- if and only if $M \leq \widehat{\theta}^{S O}$ and $\mu \in\left[c-\delta\left(S S^{s}-2 \pi_{i}^{S s}\right)+\max \left(0, \delta\left(\mu-S_{i}^{s}\right)\right), m\right]$, there exists a continuum of equilibria where firm $i$ makes a profit of $\delta \pi_{i}^{S s}>0$ by charging $p_{i}^{s}=c-\delta\left[\pi\left(R_{i}^{s}\right)-\pi_{i}^{S s}\right]$ in the first period and $S_{i}^{s}, R_{i}^{s}$ satisfying (24) in the second-period semi-niche market equilibria, where

$$
\begin{aligned}
& S S^{s}=\int_{R_{i}^{s}}^{\infty}(x-c) d F(x)+\int_{-\infty}^{R_{i}^{s}}\left[E\left(\theta_{-i} \mid x\right)-c\right] d F(x), \\
& \pi_{i}^{S s}=\left[\rho R_{i}^{S}+(1-\rho) \mu-c\right] F\left(R_{i}^{s}\right), \\
& \max (M, \mu) \leq R_{i}^{s} \leq \min \left(m, \widehat{\theta}^{S O}\right)
\end{aligned}
$$

- if and only if $\mu \in[m, \infty)$, there exists an equilibrium where firm $i$ makes a profit of $\frac{\delta(1-\rho)}{4 f(\mu)}>0$ by charging $p_{i}^{m}=c$ in the first period and $R_{i}^{m}, S_{i}^{m}$ as given by (26) in the second-period mass market equilibrium.

According to Theorem 1 in the paper, firms make positive profits in the two-stage pricing game whenever there are switchers in the second period. With negative information spillovers, consumers do not leave the market after a bad experience as they expect to have high a valuation for the untried product. Hence, they switch to that product. Those who choose to leave the market in the second period, if any, are the consumers with intermediate valuations for the good they have tried. Therefore, since a positive mass of consumers switch products in the second period in all equilibria with $\rho \in[-1,0)$, firms always make positive profits in this case.

As before, the relative profitability of switching consumers determines how intensely firms compete for market share in the first period. In the mass market case here, as when $\rho=0$, switching and repeat customers are equally profitable. This breaks the link between first and second period competition and firms compete à la Bertrand in the first period, setting price equal to marginal cost.

\section{C.3 Proofs for the negative information spillovers setting}

Proof of Lemma 1. We prove the lemma in three steps.

Step 1: The niche market equilibrium

We now prove that condition $M \geq \widehat{\theta}^{S O}$ is sufficient and necessary for the existence of a niche market equilibrium.

We start from sufficiency. We first show that when $M \geq \widehat{\theta}^{S O}$ holds, we have $M \geq \mu$. Suppose $M<\mu$, then $\mu-\frac{1}{2 f(\mu)}-c>0$. Given that $M \geq \widehat{\theta}^{S O}$ we also have $\widehat{\theta}^{S O}<\mu$, which 
implies that $\widehat{S}>\mu$, because $\widehat{\theta}^{S O}=\frac{\widehat{S}-(1-\rho) \mu}{\rho}$, and that, according to (23),

$$
\widehat{S}<\frac{-\rho}{2 f(\mu)}+c<\frac{1}{2 f(\mu)}+c
$$

But then this implies $\mu<\frac{1}{2 f(\mu)}+c$. A contradiction to $M<\mu$. Hence, we have $M \geq \mu$.

To show that the prices given in the lemma form an equilibrium, we need to rule out two sorts of profitable deviations. First, we show that among all prices such that $\theta^{R O}>\theta^{S O}$ holds, i.e., repeat and switching consumers' surplus curves cross at a point below the horizontal axis similar as in the niche market equilibrium, are no better than charging $M$ to repeat consumers and $\widehat{S}$ to switching consumers. Holding the other firm charges the equilibrium price, a firm makes the following profit from repeat consumers if it charges a price satisfies $R_{i}>\widehat{\theta}^{S O}$, i.e., the surplus curves cross below the horizontal axis:

$$
\lambda_{i}\left(R_{i}-c\right) \int_{R_{i}}^{\infty} d F(\theta)
$$

By first order condition of profit function (28) w.r.t $R_{i}$, any local deviations by firm $i$ such that the two surplus curves still cross at a point below the horizontal axis must not be more profitable than $M$, because otherwise the first order derivative w.r.t $R_{i}$ is not equal to zero. Hence, the optimal choice is that $R_{i}=M$ because the firm behaves as a monopoly in its share of the market. Alternatively, it makes the following profit from switching consumers if it charges a price satisfies $S_{i}>\rho M+(1-\rho) \mu$, or put it differently, $\theta_{-i}^{S O}=\frac{S_{i}-(1-\rho) \mu}{\rho}<M=\theta_{-i}^{R O}$, i.e., $S_{i}$ maintains a situation similar to the niche market equilibrium:

$$
\lambda_{-i}\left(S_{i}-c\right) \int_{-\infty}^{\frac{S_{i}-(1-\rho) \mu}{\rho}} d F(\theta) .
$$

The optimal price can be found by first order approach and is, in fact, given by $\widehat{S}$. Therefore, $M$ and $\widehat{S}$ are the optimal prices to repeat and switching consumers among all prices that have consumers' surplus curves cross below the horizontal axis.

Second, we show that prices that let consumer surplus curves cross above the horizontal axis are not profitable deviations. Suppose firm $i$ deviates the price to repeat consumers to a $R_{i}<\widehat{\theta}^{S O}$ such that the two surplus curves cross above the horizontal axis, holding the rival firm chooses the equilibrium prices. Then the profit from repeat customers is given by

$$
\lambda_{i}\left(R_{i}-c\right) \int_{\mu+\frac{R_{i}-\widehat{S}}{1-\rho}}^{\infty} d F(\theta),
$$


then by the first order approach the optimal price must satisfy the following condition:

$$
R_{i}^{*}=\frac{(1-\rho)\left[1-F\left(\mu+\frac{R_{i}^{*}-\widehat{S}}{1-\rho}\right)\right]}{f\left(\mu+\frac{R_{i}^{*}-\widehat{S}}{1-\rho}\right)}+c .
$$

Such an optimal is unique, as the right side of the above equation is a decreasing function of $R_{i}^{*}$ due to the MHR property. According to $R_{i}^{*}<\widehat{\theta}^{S O}$, we have

$$
R_{i}^{*}>\frac{(1-\rho)\left[1-F\left(\widehat{\theta}^{S O}\right)\right]}{f\left(\widehat{\theta}^{S O}\right)}+c>\frac{(1-\rho)[1-F(m)]}{f(m)}+c=m>M \geq \widehat{\theta}^{S O}
$$

which contradicts $R_{i}^{*}<\widehat{\theta}^{S O}$. Therefore, $R_{i}^{*} \geq \widehat{\theta}^{S O}$ and any price $R_{i}<\widehat{\theta}^{S O}$ is not a profitable deviation, because the first order derivative of the profit function (29) must be positive for any such price $R_{i}$. In other words, the firm would always prefer to increase the price whenever $R_{i}<\widehat{\theta}^{S O}$ holds.

Now suppose firm $i$ deviates to a price $S_{i}<\rho M+(1-\rho) \mu$, again holding the rival firm chooses the equilibrium prices, then the two surplus curves cross at a point above the horizontal axis. The profit from doing so is given by

$$
\lambda_{-i}\left(S_{i}-c\right) \int_{-\infty}^{\mu+\frac{M-S_{i}}{1-\rho}} d F(\theta)
$$

and hence, the optimal deviation price must satisfy

$$
S_{i}^{*}=\frac{(1-\rho) F\left(\mu+\frac{M-S_{i}^{*}}{1-\rho}\right)}{f\left(\mu+\frac{M-S_{i}^{*}}{1-\rho}\right)}+c .
$$

Since $S_{i}^{*}<\rho M+(1-\rho) \mu$ and $M \geq \mu$, we have

$$
S_{i}^{*}>\frac{(1-\rho) F(M)}{f(M)}+c \geq \frac{(1-\rho)[1-F(M)]}{f(M)}+c>\frac{1-F(M)}{f(M)}+c=M \geq \mu .
$$

But by $S_{i}^{*}<\rho M+(1-\rho) \mu$ and $M \geq \mu$, we have $S_{i}^{*} \leq \mu$. A contradiction. Hence, $S_{i}^{*} \geq$ $\rho M+(1-\rho) \mu$, or put it differently $\theta_{-i}^{S O}=\frac{S_{i}^{*}-(1-\rho) \mu}{\rho} \leq M=\theta_{-i}^{R O}$, must hold. Therefore, any price $S_{i}<\rho M+(1-\rho) \mu$ is not a profitable deviation, because it is less than the optimal price $S_{i}^{*}$. Hence, the firm would rather choose a price satisfies $S_{i} \geq \rho M+(1-\rho) \mu$. We have completed the proof of sufficiency.

Now we turn to necessity. Suppose the prices (22) and (23) form an equilibrium. Then it must be true that $M \geq \widehat{\theta}^{S O}$ for otherwise the two surplus curves would cross at a point above 
the horizontal axis.

\section{Step 2: The semi-niche market equilibria}

We start from proving sufficiency. Note that the conditions imply that

$$
\widehat{\theta}^{S O} \equiv \frac{\widehat{S}-(1-\rho) \mu}{\rho}>R_{i}^{s}>\max (\mu, M)
$$

Holding the rival firm choosing the equilibrium prices, and suppose firm $i$ charges a price $R_{i}>$ $R_{i}^{s}$. Repeat customers' surplus curve moves to the right and crosses the switching customers' surplus curve at a point below the horizontal axis. In this case, the profit from repeat customers is again given by (28) which is maximized at $M$. Since (30) requires that $R_{i}^{s}>\mu$ and the profit function above is inversely U-shaped due to our monotone hazard rate assumption, the first order derivative of firm $i$ 's profit from repeat customers at $R_{i}>R_{i}^{s}>M$ must be negative, which implies that $R_{i}$ is not a profitable deviation.

Suppose instead, firm $i$ deviates to a price $R_{i}^{\prime}<R_{i}^{s}$. Then the two surplus curves cross at a point above the horizontal axis, and the profit from repeat customers is given by

$$
\lambda_{i}\left(R_{i}^{\prime}-c\right) \int_{\mu+\frac{R_{i}^{\prime}-S_{-i}^{s}}{1-\rho}}^{\infty} d F(\theta)
$$

By the first order condition w.r.t $R_{i}^{\prime}$, the implicit best response function of firm $i$ is given by

$$
R_{i}^{* *}=\frac{(1-\rho)\left[1-F\left(\mu+\frac{R_{i}^{\prime *}-S_{-i}^{s}}{1-\rho}\right)\right]}{f\left(\mu+\frac{R_{i}^{\prime *}-S_{-i}^{s}}{1-\rho}\right)}
$$

Note that the MHR assumption guarantees that the solution to the first order condition is indeed the optimal. Suppose $R_{i}^{\prime *}<R_{i}^{s}$, then according to the above best response function,

$R_{i}^{\prime *}>\frac{(1-\rho)\left[1-F\left(\mu+\frac{R_{i}^{s}-S_{i}^{s}}{1-\rho}\right)\right]}{f\left(\mu+\frac{R_{i}^{s}-S_{i}^{s}}{1-\rho}\right)}+c=\frac{(1-\rho)\left[1-F\left(R_{i}^{s}\right)\right]}{f\left(R_{i}^{s}\right)}+c>\frac{(1-\rho)[1-F(m)]}{f(m)}+c=m>R_{i}^{s}$.

A contradiction. Thus, $R_{i}^{\prime *} \geq R_{i}^{s}$. Therefore, deviating to any price $R_{i}^{\prime}<R_{i}^{s}$ is not profitable because $R_{i}^{\prime}<R_{i}^{\prime *}$, which implies that the first order derivative of the profit w.r.t $R_{i}^{\prime}$ is positive.

Now, suppose firm $i$ charges a price $S_{i}>S_{i}^{s}$ to attract firm $-i$ 's customers. Then these switching customers' surplus curve moves left and the two surplus curves cross at a point below the horizontal axis. Firm $i$ 's profit from switching customers is then given by

$$
\lambda_{-i}\left(S_{i}-c\right) \int_{-\infty}^{\frac{S_{i}-(1-\rho) \mu}{\rho}} d F(\theta)
$$


and the optimal price is in fact $\widehat{S}$. According to (30) and (24), which imply that

$$
\widehat{S}<\rho R_{-i}^{s}+(1-\rho) \mu=S_{i}^{s}<\mu,
$$

we have $S_{i}<S_{i}^{s}<\widehat{S}$, and hence, the first order derivative of firm $i$ 's profit w.r.t $S_{i}$ must be positive. Thus, any $S_{i}<S_{i}^{s}$ is not profitable to deviate to.

Suppose instead, firm $-i$ deviates to a price $q_{-i}^{\prime}<S_{-i}^{s}$. The switching customers' surplus curve must now cross the repeat customers' surplus curve at a point above the horizontal axis. The profit from the switching customers are then given by

$$
\left(S_{i}^{\prime}-c\right) \int_{-\infty}^{\mu+\frac{R_{-i}^{s}-S_{i}^{\prime}}{1-\rho}} d F(\theta)
$$

By the first order approach, firm $-i$ 's implicit best response function is given by

$$
S_{i}^{\prime *}=\frac{(1-\rho) F\left(\mu+\frac{R_{-i}^{s}-S_{i}^{\prime *}}{1-\rho}\right)}{f\left(\mu+\frac{R_{-i}^{s}-S_{i}^{\prime *}}{1-\rho}\right)}+c .
$$

Suppose $S_{i}^{\prime *}<\widehat{S}$, then

$$
S_{i}^{*}>\frac{(1-\rho) F\left(R_{-i}^{s}\right)}{f\left(R_{-i}^{s}\right)}+c>\frac{1-\rho}{2 f(\mu)}+c \geq \mu
$$

However, (30) and (24) imply that $\widehat{S}<\mu$. A contradiction. Hence, $S_{i}^{*} \geq \widehat{S}$ and it is not profitable to deviate to $S_{i}^{\prime}<S_{i}^{s}$ since the first order derivative of firm $i$ 's profit from switching customers w.r.t $S_{i}^{\prime}$ must be positive.

\section{Step 3: The mass market equilibrium}

We can solve for the equilibrium prices from taking first order conditions w.r.t $R_{i}$ and $S_{i}$ of the profit function of firm $i$ in mass market equilibrium given below

$$
\lambda_{i}\left(R_{i}-c\right) \int_{\theta_{i}^{R S}}^{+\infty} d F\left(\theta_{i}\right)+\lambda_{-i}\left(S_{i}-c\right) \int_{-\infty}^{\theta_{-i}^{R S}} d F\left(\theta_{-i}\right) .
$$

The profit function (31) is obtained by letting $\max \left(\theta_{i}^{R S}, \theta_{i}^{R O}\right)=\theta_{i}^{R S}$ and $\min \left(\theta_{-i}^{R S}, \theta_{-i}^{S O}\right)=\theta_{-i}^{R S}$ in (21). We then obtain the following necessary conditions for equilibrium prices:

$$
R_{i}^{m}=\frac{(1-\rho)\left[1-F\left(\theta_{i}^{R S}\right)\right]}{f\left(\theta_{i}^{R S}\right)}+c \quad \text { and } \quad S_{i}^{m}=\frac{(1-\rho) F\left(\theta_{-i}^{R S}\right)}{f\left(\theta_{-i}^{R S}\right)}+c .
$$


We can find the cutoff types for any $\lambda_{i}$ :

$$
(1-\rho)\left(\theta_{i}^{R S}-\mu\right)=R_{i}^{m}-S_{-i}^{m}=\frac{(1-\rho)\left[1-2 F\left(\theta_{i}^{R S}\right)\right]}{f\left(\theta_{i}^{R S}\right)}
$$

hence,

$$
\theta_{i}^{R S}=\mu+\frac{1-2 F\left(\theta_{i}^{R S}\right)}{f\left(\theta_{i}^{R S}\right)}
$$

Suppose $\theta_{i}^{R S}<\mu$, then $\frac{1-2 F\left(\theta_{i}^{R S}\right)}{f\left(\theta_{i}^{R S}\right)}>0$ and hence the RHS of (33) must be greater than $\mu$, which is a contradiction. Suppose $\theta_{i}^{R S}>\mu$, then $\frac{1-2 F\left(\theta_{i}^{R S}\right)}{f\left(\theta_{i}^{R S}\right)}<0$ and hence the RHS of (33) must be less than $\mu$. This means that it must be true that $\theta_{i}^{R S}=\mu$, where $i \in\{A, B\}$. Plugging $\theta^{R S}=\mu$ back into the two equations for $R_{i}^{m}$ and $S_{i}^{m}$, respectively, we obtain the mass market equilibrium prices.

The above analysis is valid if and only if the prices indeed form a mass market equilibrium, i.e., $\theta^{R S}-R_{i}^{m} \geq 0$ is true. Hence, the sufficient and necessary condition for the existence of the above mass market equilibrium is:

$$
\mu-\frac{1-\rho}{2 f(\mu)}-c \geq 0
$$

or equivalently, $m \leq \mu$.

Proof of Corollary \%. We prove the corollary in two steps.

Step 1. The fact that the niche market equilibrium exists implies that $M \geq \widehat{\theta}^{S O}$ and $M \geq \mu$.

Claim 1: When $\rho=-1, M=\widehat{S} \geq \mu$ and $\pi(M)=\pi_{i}^{S n}$.

First, note that when $\rho=-1$, it is true that:

$$
\begin{aligned}
R_{i}^{n} & =M=\frac{1-F(M)}{f(M)}+c, \\
S_{i}^{n} & =\widehat{S}=\frac{F(2 \mu-\widehat{S})}{f(2 \mu-\widehat{S})}+c .
\end{aligned}
$$

We can rewrite $\widehat{S}$ as

$$
\widehat{S}=\frac{F(2 \mu-\widehat{S})}{f(2 \mu-\widehat{S})}+c=\frac{F(\mu-(\widehat{S}-\mu))}{f(\mu-(\widehat{S}-\mu))}+c=\frac{1-F(\mu+(\widehat{S}-\mu))}{f(\mu+(\widehat{S}-\mu))}+c=\frac{1-F(\widehat{S})}{f(\widehat{S})}+c
$$

This implies that $R_{i}^{n}=\widehat{S}=M \geq \mu$ and $\pi(M)=\pi_{i}^{S n}$ when $\rho=-1$. 
Claim 2: When $\rho \in[-1,0), \widehat{S} \leq M$.

Take the first order derivative of (23)'s RHS w.r.t $\rho$ :

$$
\frac{d \widehat{S}}{d \rho}=-\frac{F\left(\widehat{\theta}^{S O}\right)}{f\left(\widehat{\theta}^{S O}\right)}-\frac{d\left(\frac{F\left(\widehat{\theta}^{S O}\right)}{f\left(\widehat{\theta}^{S O}\right)}\right)}{d \widehat{\theta}^{S O}} \frac{d \widehat{S}}{d \rho}-\frac{d\left(\frac{F\left(\widehat{\theta}^{S O}\right)}{f\left(\widehat{\theta}^{S O}\right)}\right)}{d \widehat{\theta}^{S O}} \frac{\mu-\widehat{S}}{\rho}
$$

and rearrange

$$
[1+\underbrace{\frac{d\left(\frac{F\left(\widehat{\theta}^{S O}\right)}{f\left(\widehat{\theta}^{S O}\right)}\right)}{d \widehat{\theta}^{S O}}}_{>0}] \frac{d \widehat{S}}{d \rho}=-\frac{F\left(\widehat{\theta}^{S O}\right)}{f\left(\widehat{\theta}^{S O}\right)}-\frac{d\left(\frac{F\left(\widehat{\theta}^{S O}\right)}{f\left(\widehat{\theta}^{S O}\right)}\right)}{d \widehat{\theta}^{S O}} \frac{\mu-\widehat{S}}{\rho} .
$$

Hence, $\widehat{S}$ decreases in $\rho$ as long as $\widehat{S} \geq \mu$. Since $\widehat{S}=M$ when $\rho=-1$ according to Claim 1 , there exists a $\rho^{\prime}>-1$ such that $\mu \leq \widehat{S} \leq M$ is true for any $\rho \in\left[-1, \rho^{\prime}\right]$.

For $\rho \in\left(\rho^{\prime}, 0\right), \frac{d \widehat{S}}{d \rho}<0$ is not necessarily true, as $\widehat{S}<\mu$ may hold. Denote by $\rho^{\prime \prime} \equiv \sup \{\rho \mid \widehat{S} \geq$ $\left.\mu, \rho \geq \rho^{\prime}\right\}$ the smallest $\rho$ such that $\widehat{S} \geq \mu$. Then for any $\rho \in\left(\rho^{\prime \prime}, 0\right)$, we have $\widehat{S} \leq \mu$, because whenever $\widehat{S}$ increases to a level sufficiently close to $\mu, \frac{d \widehat{S}}{d \rho}<0$ holds and $\widehat{S}$ starts to decrease. Therefore, $\widehat{S} \leq M$ holds for all $\rho \in[-1,0)$. See the following figure for this part of the proof.

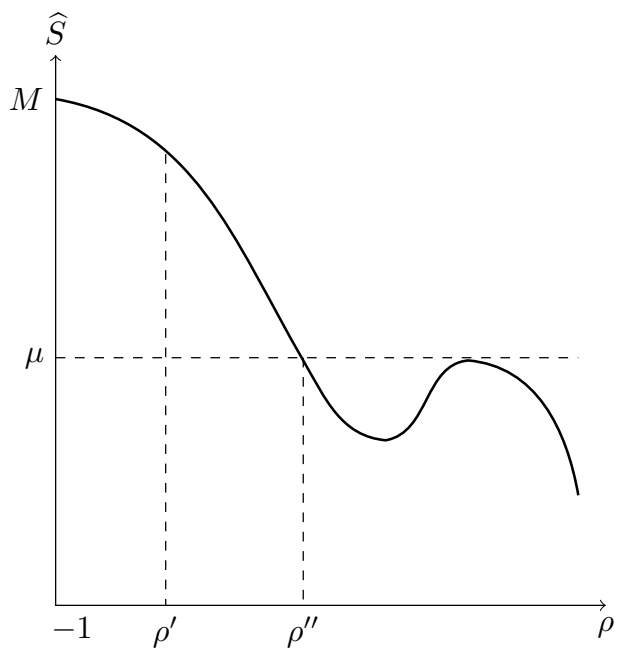

Figure C.5: $\widehat{S} \leq M$ holds for all $\rho \in[-1,0)$.

Step 2. According to Lemma 1, the sufficient and necessary condition for the semi-niche market implies $R_{i}^{s} \geq \mu$. Since $S_{i}^{s}=\rho R_{i}^{s}+(1-\rho) \mu$ and $\rho<0$, it is true that $S_{i}^{s} \leq \mu$ and hence, $S_{i}^{s} \leq R_{i}^{s}$

Proof of Proposition \%. This proof has three steps.

Step 1: The equilibrium when the second-period sub-game has the mass market equilibrium 
Since the second-period prices and the profits are the same across repeat and switching consumers, firms are indifferent across any market share in the second period. Hence, they compete in the first period only for the first-period profit. This implies that they behave as if they are in Bertrand competition by charging the marginal cost $c$. In that case, consumer surplus must be non-negative since $\mu-c \geq m-c \geq S_{i}^{m}-c=R_{i}^{m}-c=\frac{1-\rho}{2 f(\mu)}>0$, i.e., consumers' first-period surplus is positive according to the condition for existence of the mass market equilibrium, and their second-period surplus must be non-negative. Furthermore, their first period surplus $\mu-c$ is greater than the surplus from only purchase in the second period, given by $\mu-S_{i}^{m}=\mu-\frac{1-\rho}{2 f(\mu)}-c$. Therefore, the sufficient and necessary condition is equivalent to the condition for existence of the mass market equilibrium in the second period.

Note that for each firm selling only in the second period is weakly dominated by selling in both periods. To see why, consider firm $i$ sells only in the second period while firm $-i$ sells in both periods. Then it must hold that $\lambda_{i}=0$ and firm $i$ 's profit is given by $\left(S_{i}^{m}-c\right) \cdot F(\mu)=$ $\frac{\delta(1-\rho)}{4 f(\mu)}$, which is equivalent to the equilibrium profit. On the other hand, consider firm $i$ sells only in the second period while firm $-i$ does the same. Then both firms make zero profit as they end up in Bertrand competition. Therefore, selling only in the second period is weakly dominated.

Step 2: The equilibrium when the second-period sub-game has the semi-niche market equilibria

Given any equilibrium in a semi-niche market, we can find the profit firm $i$ makes from repeat customers, $\left(R_{i}^{s}-c\right)\left[1-F\left(R_{i}^{s}\right)\right]$, and from switching customers, $\left[\rho R_{i}^{s}+(1-\rho) \mu-c\right] F\left(R_{i}^{s}\right)$, with $\max (M, \mu) \leq R_{i}^{s} \leq \min \left(m, \widehat{\theta}^{S O}\right)$.

In Theorem 1 in the paper we have shown that each firm's profit in the whole game is given by $\delta \pi_{i}^{S *}$. The following derivation illustrates how this holds in the current context. When $\pi\left(R_{i}^{s}\right) \geq \pi_{i}^{S s}$ firms make greater profit from repeat customers than from switchers. Firms then compete for market share in the first period by lowering price to the point where $c-p_{i}^{s}=\delta\left(\pi\left(R_{i}^{s}\right)-\pi_{i}^{S s}\right)$. Suppose firm $i$ deviates to a higher price in the first period, then it avoids the loss $\lambda_{i}\left(c-p_{i}^{s}\right)$ in the first period, but its profit in the second period also decreases by $\delta \lambda_{i}\left(\pi\left(R_{i}^{s}\right)-\pi_{i}^{S s}\right)$. Suppose it deviates to a slightly lower price in the first period, then it obtains a gain of $\delta \lambda_{-i}\left(\pi\left(R_{i}^{s}\right)-\pi_{i}^{S s}\right)$ but also incurs an additional loss of $\lambda_{-i}\left(c-p_{i}^{s}\right)$ in the second period. Hence, neither deviating upwards or downwards is profitable.

Alternatively, when $\pi\left(R_{i}^{s}\right)<\pi_{i}^{S s}$ firms make greater profit from switchers than from repeat customers. Firms then compete for lower market share by increasing price up to $p_{i}^{s}-c=$ $\delta\left(\pi_{i}^{S s}-\pi\left(R_{i}^{s}\right)\right)$. Suppose firm $i$ deviates to a higher price in the first period, then it loses profits 
$\lambda_{i}\left(p_{i}^{s}-c\right)$ in the first period, but its profits in the second period increase by $\delta \lambda_{i}\left(\pi_{i}^{S s}-\pi\left(R_{i}^{s}\right)\right)$. Suppose it deviates to a slightly lower price in the first period, then it makes an additional profit of $\lambda_{-i}\left(p_{i}^{s}-c\right)$, but loses $\delta \lambda_{-i}\left(\pi_{i}^{S s}-\pi\left(R_{i}^{s}\right)\right)$ in the second period. Hence, neither deviating upwards or downwards is profitable. Firm $i$ 's total discounted profit over the two periods is thus $\lambda_{i}\left(p_{i}^{s}-c\right)+\delta\left(\lambda_{i} \pi\left(R_{i}^{s}\right)+\lambda_{-i} \pi_{i}^{S s}\right)=\delta \pi_{i}^{S s}$.

Following a similar procedure as in step 1, it can be shown that selling only in the second period is weakly dominated for each firm.

Consumers' surplus from purchasing good $i$ in the first period is given by

$$
\begin{aligned}
& \left(\mu-p_{i}^{s}\right)+\delta\left[\int_{R_{i}^{s}}^{\infty}\left(x-R_{i}^{s}\right) d F(x)+\int_{-\infty}^{R_{i}^{s}}\left[E\left(\theta_{-i} \mid x\right)-S_{-i}^{s}\right] d F(x)\right] \\
= & (\mu-c)+\delta\left(\pi\left(R_{i}^{s}\right)-\pi_{i}^{S s}\right)+\delta\left[\int_{R_{i}^{s}}^{\infty}(x-c) d F(x)-\pi\left(R_{i}^{s}\right)+\int_{-\infty}^{R_{i}^{s}}\left[E\left(\theta_{-i} \mid x\right)-c\right] d F(x)-\pi_{i}^{S s}\right] \\
= & (\mu-c)+\delta[\underbrace{\int_{R_{i}^{s}}^{\infty}(x-c) d F(x)+\int_{-\infty}^{R_{i}^{s}}\left[E\left(\theta_{-i} \mid x\right)-c\right] d F(x)}_{S S^{s}}-2 \pi_{i}^{S s}]
\end{aligned}
$$

Hence, the above consumer surplus is non-negative if and only if $\mu \geq c-\delta \cdot\left(S S^{s}-2 \pi_{i}^{S s}\right)$ and is greater than the surplus from purchasing only in the second period if and only if $\mu \geq$ $c-\delta \cdot\left(S S^{s}-2 \pi_{i}^{S s}\right)+\delta\left(\mu-S_{i}^{s}\right)$. Combining the two inequalities yields the lower bound of the sufficient and necessary condition given in the proposition.

Step 3: The equilibrium when the second-period sub-game has the niche market equilibrium

Suppose firm $i$ deviates to a price higher than $p_{i}^{n}$. Then profits in the first period increase by $\lambda_{i}\left(c-p_{i}^{n}\right)$ to 0 . The discounted profits in the second period decrease by $\lambda_{i} \pi(M)-\lambda_{i} \pi_{i}^{S n}$ to $\pi_{i}^{S n}$. Since the gain is the same as the loss, it is not a profitable deviation. Suppose firm $i$ deviates to a slightly lower price $p_{i}^{n}-\epsilon$, then the profits in the first period decrease by $\lambda_{-i}\left(c-p_{i}^{n}\right)$ to $c-p_{i}^{n}$. The discounted profits in the second period increase by $\lambda_{-i} \pi(M)-\lambda_{-i} \pi_{i}^{S n}$. Thus, the gain is the same as the loss and it is not a profitable deviation.

Following a similar procedure as in step 1, it can be shown that selling only in the second period is weakly dominated for each firm.

The last question left to answer is whether consumers are willing to buy one of the products in the market in the first period given the equilibrium prices. Consumer surplus can be calculated 
by accounting for the equilibrium prices:

$$
\begin{aligned}
& \left(\mu-p_{i}^{n}\right)+\delta\left[\int_{M}^{\infty}(x-M) d F(x)+\int_{-\infty}^{\widehat{\theta}^{S O}}\left[E\left(\theta_{-i} \mid x\right)-\widehat{S}\right] d F(x)\right] \\
= & (\mu-c)+\delta\left(\pi(M)-\pi_{i}^{S n}\right)+\delta\left[\int_{M}^{\infty}(x-c) d F(x)-\pi(M)+\int_{-\infty}^{\widehat{\theta}^{S O}}\left[E\left(\theta_{-i} \mid x\right)-c\right] d F(x)-\pi_{i}^{S n}\right] \\
= & (\mu-c)+\delta[\underbrace{\int_{M}^{\infty}(x-c) d F(x)+\int_{-\infty}^{\widehat{\theta}^{S O}}\left[E\left(\theta_{-i} \mid x\right)-c\right] d F(x)}_{S S^{n}}-2 \pi_{i}^{S n}]
\end{aligned}
$$

Hence, the above consumer surplus to is non-negative if and only if $\mu \geq c-\delta\left(S S^{n}-2 \pi_{i}^{S n}\right)$ and is greater than the surplus from purchasing only in the second period if and only if $\mu \geq$ $c-\delta\left(S S^{n}-2 \pi_{i}^{S n}\right)+\delta \cdot(\mu-\widehat{S})$. Combining the two inequalities yields the lower bound of the sufficient and necessary condition given in the proposition.

\section{Price elasticities}

\section{D.1 When $\rho \in(0,1)$}

Denote by $D_{i}^{R}$ the demand from repeat consumers of firm $i$, and by $D_{i}^{S}$ the demand from switching consumers of firm $i$. In general,

$$
\begin{aligned}
D_{i}^{R} & =\lambda_{i} \int_{\max \left(\theta_{i}^{R S}, \theta_{i}^{R O}\right)}^{+\infty} d F\left(\theta_{i}\right) \\
D_{i}^{S} & =\max \left(0, \lambda_{-i} \int_{\theta_{-i}^{S O}}^{\theta_{-i}^{R S}} d F\left(\theta_{-i}\right)\right) .
\end{aligned}
$$

In a mass market equilibrium, $D_{i}^{R}=\lambda_{i} \int_{\theta_{i}^{R S}}^{+\infty} d F\left(\theta_{i}\right)$ and $D_{i}^{S}=\lambda_{-i} \int_{\theta_{-i}^{S O}}^{\theta_{-i}^{R S}} d F\left(\theta_{-i}\right)$. Note that $D_{i}^{R}$ is a function of $R_{i}^{m}$ and $S_{-i}^{m}$ as $\theta_{i}^{R S}$ is determined according to $\mu+\left(R_{i}^{m}-S_{-i}^{m}\right) /(1-\rho)$, and that $D_{i}^{S}$ is a function of $R_{-i}^{m}$ and $S_{i}^{m}$ as $\theta_{-i}^{R S}$ is determined by $\mu+\left(R_{-i}^{m}-S_{i}^{m}\right) /(1-\rho)$ and $\theta_{-i}^{S O}$ is determined by $\left(S_{i}^{m}-(1-\rho) \mu\right) / \rho$. 
The relevant elasticities are then given by

$$
\begin{aligned}
E_{R_{i}^{m}}^{D^{R}} & =\frac{d D_{i}^{R}}{d R_{i}^{m}} \frac{R_{i}^{m}}{D_{i}^{R}}=-\frac{\lambda_{i} f\left(\theta_{i}^{R S}\right)}{1-\rho} \cdot \frac{R_{i}^{m}}{\lambda_{i}\left[1-F\left(\theta_{i}^{R S}\right)\right]}=-\frac{R_{i}^{m}}{R_{i}^{m}-c} \\
E_{S_{-i}^{m}}^{D_{i}^{R}} & =\frac{d D_{i}^{R}}{d S_{-i}^{m}} \frac{S_{-i}^{m}}{D_{i}^{R}}=\frac{\lambda_{i} f\left(\theta_{i}^{R S}\right)}{1-\rho} \cdot \frac{S_{-i}^{m}}{\lambda_{i}\left[1-F\left(\theta_{i}^{R S}\right)\right]}=\frac{S_{-i}^{m}}{R_{i}^{m}-c} \\
E_{R_{-i}^{m}}^{D_{i}^{S}} & =\frac{d D_{i}^{S}}{d R_{-i}^{m}} \frac{R_{-i}^{m}}{D_{i}^{S}}=\frac{\lambda_{i} f\left(\theta_{-i}^{R S}\right)}{1-\rho} \cdot \frac{R_{-i}^{m}}{\lambda_{i}\left[F\left(\theta_{-i}^{R S}\right)-F\left(\theta_{-i}^{S O}\right)\right]}=\frac{f\left(\theta_{-i}^{R S}\right) R_{-i}^{m}}{(1-\rho)\left[F\left(\theta_{-i}^{R S}\right)-F\left(\theta_{-i}^{S O}\right)\right]} \\
E_{S_{i}^{m}}^{D^{S}} & =\frac{d D_{i}^{S}}{d S_{i}^{m}} \frac{S_{i}^{m}}{D_{i}^{S}}=-\lambda_{-i}\left[\frac{f\left(\theta_{-i}^{R S}\right)}{1-\rho}+\frac{f\left(\theta_{-i}^{S O}\right)}{\rho}\right] \cdot \frac{S_{i}^{m}}{\lambda_{-i}\left[F\left(\theta_{-i}^{R S}\right)-F\left(\theta_{-i}^{S O}\right)\right]}=-\frac{S_{i}^{m}}{S_{i}^{m}-c} .
\end{aligned}
$$

In a niche market equilibrium, $D_{i}^{S}=0$ and hence, the relevant elasticities are zero: $E_{R_{-i}^{n}}^{D_{i}^{S}}=$ $E_{S_{i}^{n}}^{D_{i}^{S}}=0$. We also have $D_{i}^{R}=\lambda_{i}\left[1-F\left(\theta_{i}^{R O}\right)\right]$ and hence,

$$
\begin{aligned}
E_{R_{i}^{n}}^{D^{R}} & =\frac{d D_{i}^{R}}{d R_{i}^{n}} \frac{R_{i}^{n}}{D_{i}^{R}}=-\frac{\lambda_{i} f\left(R_{i}^{n}\right) R_{i}^{n}}{\lambda_{i}\left[1-F\left(R_{i}^{n}\right)\right]}=-\frac{M}{M-c} \\
E_{S_{-i}^{n}}^{D_{i}^{R}} & =0 .
\end{aligned}
$$

In a semi-niche market equilibrium, the demand curves are kinked:

$$
\begin{gathered}
D_{i}^{R}=\left\{\begin{array}{l}
\lambda_{i}\left[1-F\left(R_{i}\right)\right], \text { if } R_{i} \leq R_{i}^{s} \\
\lambda_{i}\left[1-F\left(\theta_{i}^{R S}\right)\right], \text { if } R_{i}>R_{i}^{s}
\end{array}=\left\{\begin{array}{l}
\lambda_{i}\left[1-F\left(R_{i}^{S}\right)\right], \text { if } S_{-i} \geq S_{-i}^{s} \\
\lambda_{i}\left[1-F\left(\theta_{i}^{R S}\right)\right], \text { if } S_{-i}<S_{-i}^{s}
\end{array}\right.\right. \\
D_{i}^{S}=\left\{\begin{array}{l}
0, \text { if } R_{-i} \leq R_{-i}^{s} \\
\lambda_{-i}\left[F\left(\theta_{-i}^{R S}\right)-F\left(\theta_{-i}^{S O}\right)\right], \text { if } R_{-i}>R_{-i}^{s}
\end{array}=\left\{\begin{array}{l}
0, \text { if } S_{i} \geq S_{i}^{s} \\
\lambda_{-i}\left[F\left(\theta_{-i}^{R S}\right)-F\left(\theta_{-i}^{S O}\right)\right], \text { if } S_{i}<S_{i}^{s}
\end{array}\right.\right.
\end{gathered}
$$

Hence, the corresponding elasticities are given by

$$
\begin{aligned}
& E_{R_{i}}^{D_{i}^{R}}=\frac{d D_{i}^{R}}{d R_{i}} \frac{R_{i}}{D_{i}^{R}}=\left\{\begin{array}{l}
-\frac{f\left(R_{i}\right) R_{i}}{1-F\left(R_{i}\right)}, \text { if } R_{i} \leq R_{i}^{s} \\
-\frac{f\left(\theta_{i}^{R S}\right) R_{i}}{(1-\rho)\left[1-F\left(\theta_{i}^{R S}\right)\right]}, \text { if } R_{i}>R_{i}^{s}
\end{array}\right. \\
& E_{S_{-i}}^{D_{i}^{R}}=\frac{d D_{i}^{R}}{d S_{-i}} \frac{S_{-i}}{D_{i}^{R}}=\left\{\begin{array}{l}
0, \text { if } S_{-i} \geq S_{-i}^{s} \\
\frac{f\left(\theta_{i}^{R S}\right) S_{-i}}{(1-\rho)\left[1-F\left(\theta_{i}^{R S}\right)\right]}, \text { if } S_{-i}<S_{-i}^{s}
\end{array}\right. \\
& E_{R_{-i}}^{D_{i}^{S}}=\frac{d D_{i}^{S}}{d R_{-i}} \frac{R_{-i}}{D_{i}^{S}}=\left\{\begin{array}{l}
0 \text { if } R_{-i} \leq R_{-i}^{s} \\
\frac{f\left(\theta_{-i}^{R S}\right) R_{-i}}{(1-\rho)\left[F\left(\theta_{-i}^{R S}\right)-F\left(\theta_{-i}^{S O}\right)\right]}, \text { if } R_{-i}>R_{-i}^{s}
\end{array}\right. \\
& E_{S_{i}}^{D_{i}^{S}}=\frac{d D_{i}^{S}}{d S_{i}} \frac{S_{i}}{D_{i}^{S}}=\left\{\begin{array}{l}
0, \text { if } S_{i} \geq S_{i}^{s} \\
{\left[\frac{f\left(\theta_{-i}^{R S}\right)}{1-\rho}+\frac{f\left(\theta_{-i}^{S O}\right)}{\rho}\right] S_{i}} \\
-\frac{F\left(\theta_{-i}^{R S}\right)-F\left(\theta_{-i}^{S O}\right)}{R S} \text { if } S_{i}<S_{i}^{s}
\end{array}\right.
\end{aligned}
$$




\section{D.2 When $\rho \in(-1,0]$}

The demand function of firm $i$ consists of two parts, the demand from repeat and from switching consumers:

$$
\begin{aligned}
D_{i}^{R} & =\lambda_{i} \int_{\max \left(\theta_{i}^{R S}, \theta_{i}^{R O}\right)}^{+\infty} d F\left(\theta_{i}\right) \\
D_{i}^{S} & =\lambda_{-i} \int_{\infty}^{\max \left(\theta_{-i}^{R S}, \theta_{-i}^{S O}\right)} d F\left(\theta_{-i}\right) .
\end{aligned}
$$

In the mass market equilibrium, we have $D_{i}^{R}=\lambda_{i} \int_{\theta_{i}^{R S}}^{+\infty} d F\left(\theta_{i}\right)$ and $D_{i}^{S}=\lambda_{-i} \int_{-\infty}^{\theta_{-i}^{R S}} d F\left(\theta_{i}\right)$. Then, the price elasticities are:

$$
\begin{aligned}
E_{R_{i}^{m}}^{D_{i}^{R}} & =\frac{d D_{i}^{R}}{d R_{i}^{m}} \frac{R_{i}^{m}}{D_{i}^{R}}=-\frac{\lambda_{i} f\left(\theta_{i}^{R S}\right)}{1-\rho} \frac{R_{i}^{m}}{\lambda_{i}\left[1-F\left(\theta_{i}^{R S}\right)\right]}=-\frac{R_{i}^{m}}{R_{i}^{m}-c} \\
E_{S_{-i}^{m}}^{D_{i}^{R}} & =\frac{d D_{i}^{R}}{d S_{-i}^{m}} \frac{S_{-i}^{m}}{D_{i}^{R}}=\frac{\lambda_{i} f\left(\theta_{i}^{R S}\right)}{1-\rho} \frac{S_{-i}^{m}}{\lambda_{i}\left[1-F\left(\theta_{i}^{R S}\right)\right]}=\frac{S_{-i}^{m}}{R_{i}^{m}-c} \\
E_{S_{i}^{m}}^{D_{i}^{S}} & =\frac{d D_{i}^{S}}{d S_{i}^{m}} \frac{S_{i}^{m}}{D_{i}^{S}}=-\frac{\lambda_{-i} f\left(\theta_{-i}^{R S}\right)}{1-\rho} \frac{S_{i}^{m}}{\lambda_{-i} F\left(\theta_{-i}^{R S}\right)}=-\frac{S_{i}^{m}}{S_{i}^{m}-c} \\
E_{R_{-i}^{m}}^{D_{i}^{S}} & =\frac{d D_{i}^{S}}{d R_{-i}^{m}} \frac{R_{-i}^{m}}{D_{i}^{S}}=\frac{\lambda_{-i} f\left(\theta_{-i}^{R S}\right)}{1-\rho} \frac{R_{-i}^{m}}{\lambda_{-i} F\left(\theta_{-i}^{R S}\right)}=\frac{R_{-i}^{m}}{S_{i}^{m}-c}
\end{aligned}
$$

We have shown that in equilibrium $R_{i}^{m}=R_{-i}^{m}=S_{i}^{m}=S_{-i}^{m}$, thus, $E_{R_{i}^{m}}^{D_{i}^{R}}=E_{S_{i}^{m}}^{D_{i}^{S}}=-E_{S_{-i}^{m}}^{D_{i}^{R}}=$ $-E_{R_{-i}^{m}}^{D^{S}}$.

Alternatively, in the niche market equilibrium, $D_{i}^{R}=\lambda_{i} \int_{\theta_{i}^{R O}}^{+\infty} d F\left(\theta_{i}\right)$ and $D_{i}^{S}=\lambda_{-i} \int_{-\infty}^{\theta_{-i}^{S O}} d F\left(\theta_{i}\right)$. The elasticities are given by:

$$
\begin{aligned}
E_{R_{i}^{n}}^{D_{i}^{R}} & =\frac{d D_{i}^{R}}{d R_{i}^{n}} \frac{R_{i}^{n}}{D_{i}^{R}}=-\lambda_{i} f\left(\theta_{i}^{R O}\right) \frac{R_{i}^{n}}{\lambda_{i}\left[1-F\left(\theta_{i}^{R O}\right)\right]}=-\frac{M}{M-c} \\
E_{S_{-i}^{n}}^{D_{i}^{R}} & =\frac{d D_{i}^{R}}{d S_{-i}^{n}} \frac{S_{-i}^{n}}{D_{i}^{R}}=0 \\
E_{S_{i}^{n}}^{D_{i}^{S}} & =\frac{d D_{i}^{S}}{d S_{i}^{n}} \frac{S_{i}^{n}}{D_{i}^{S}}=\frac{\lambda_{i} f\left(\theta_{-i}^{S O}\right)}{\rho} \frac{S_{i}^{n}}{\lambda_{-i} F\left(\theta_{-i}^{S O}\right)}=-\frac{S_{i}^{n}}{S_{i}^{n}-c} \\
E_{R_{-i}^{n}}^{D_{i}^{S}} & =\frac{d D_{i}^{S}}{d R_{-i}^{n}} \frac{R_{-i}^{n}}{D_{i}^{S}}=0 .
\end{aligned}
$$

In a semi-niche equilibrium, the demand curves are kinked:

$$
D_{i}^{R}=\left\{\begin{array}{l}
\lambda_{i}\left[1-F\left(R_{i}\right)\right], \text { if } R_{i} \geq R_{i}^{s} \\
\lambda_{i}\left[1-F\left(\theta_{i}^{R S}\right)\right], \text { if } R_{i}<R_{i}^{s}
\end{array}=\left\{\begin{array}{l}
\lambda_{i}\left[1-F\left(R_{i}^{s}\right)\right], \text { if } S_{-i} \geq S_{-i}^{s} \\
\lambda_{i}\left[1-F\left(\theta_{i}^{R S}\right)\right], \text { if } S_{-i}<S_{-i}^{s}
\end{array}\right.\right.
$$




$$
D_{i}^{S}=\left\{\begin{array}{l}
\lambda_{-i} F\left(\frac{S_{i}^{n}-(1-\rho) \mu}{\rho}\right), \text { if } R_{-i} \geq R_{-i}^{s} \\
\lambda_{-i} F\left(\theta_{-i}^{R S}\right), \text { if } R_{-i}<R_{-i}^{s}
\end{array}=\left\{\begin{array}{l}
\lambda_{-i} F\left(\frac{S_{i}-(1-\rho) \mu}{\rho}\right), \text { if } S_{i} \geq S_{i}^{s} \\
\lambda_{-i} F\left(\theta_{-i}^{R S}\right), \text { if } S_{i}<S_{i}^{s}
\end{array}\right.\right.
$$

Hence, the corresponding elasticities are given by

$$
\begin{aligned}
& E_{R_{i}}^{D_{i}^{R}}=\frac{d D_{i}^{R}}{d R_{i}} \frac{R_{i}}{D_{i}^{R}}=\left\{\begin{array}{l}
-\frac{f\left(R_{i}\right) R_{i}}{1-F\left(R_{i}\right)}, \text { if } R_{i} \geq R_{i}^{s} \\
-\frac{f\left(\theta_{i}^{R S}\right) R_{i}}{(1-\rho)\left[1-F\left(\theta_{i}^{R S}\right)\right]}, \text { if } R_{i}<R_{i}^{s}
\end{array}\right. \\
& E_{S_{-i} D_{i}^{R}}=\frac{d D_{i}^{R}}{d S_{-i}} \frac{S_{-i}}{D_{i}^{R}}=\left\{\begin{array}{l}
0, \text { if } S_{-i} \geq S_{-i}^{s} \\
\frac{f\left(\theta_{i}^{R S}\right) S_{-i}}{(1-\rho)\left[1-F\left(\theta_{i}^{R S}\right)\right]}, \text { if } S_{-i}<S_{-i}^{s}
\end{array}\right. \\
& E_{R_{-i}}^{D_{i}^{S}}=\frac{d D_{i}^{S}}{d R_{-i}} \frac{R_{-i}}{D_{i}^{S}}=\left\{\begin{array}{c}
0, \text { if } R_{-i} \geq R_{-i}^{s} \\
\frac{f\left(\theta_{-i}^{R S}\right) R_{-i}}{(1-\rho) F\left(\theta_{-i}^{R S}\right)}, \text { if } R_{-i}<R_{-i}^{s}
\end{array}\right. \\
& E_{S_{i} D_{i}^{S}}=\frac{d D_{i}^{S}}{d S_{i}} \frac{S_{i}}{D_{i}^{S}}=\left\{\begin{array}{l}
\frac{f\left(\frac{S_{i}-(1-\rho) \mu}{\rho}\right) S_{i}}{\rho F\left(\frac{S_{i}-(1-\rho) \mu}{\rho}\right)}, \text { if } S_{i} \geq S_{i}^{s} \\
-\frac{f\left(\theta_{-i}^{S O}\right) S_{i}}{(1-\rho) F\left(\theta_{-i}^{R S}\right)}, \text { if } S_{i}<S_{i}^{s}
\end{array}\right.
\end{aligned}
$$

\section{E Competitive pricing of normal goods: A benchmark}

In our main analysis, we provide comparative statics regarding how firm profits and consumer surplus vary with the degree of information spillovers between experience goods. In this appendix, we provide a benchmark case of the analysis of a two-period price competition in a normal goods market, of which the comparison with the main analysis can help to illustrate the effect of the informational switching cost.

Consumers know their WTP for both normal goods, whereas firms only know the joint (bi-normal) distribution of the WTPs, denoted by $f\left(\theta_{A}, \theta_{B} ; \rho\right)$. This model is equivalent to our main model except that consumers do not have to learn about their WTPs - they are fully aware of their WTPs from the start of the first period.

Denote the single-period price of firms by $p_{A}, p_{B}$. Then, the probability that a consumer purchases from firm $A$ is

$$
\operatorname{Pr}\left(\theta_{A}-\theta_{B} \geq p_{A}-p_{B}, \theta_{A}-p_{A} \geq 0\right)
$$


Hence, the single-period profit of firm $A$ is

$$
\begin{aligned}
& \left(p_{A}-c\right) \int_{p_{A}}^{+\infty} \int_{-\infty}^{\theta_{A}-p_{A}+p_{B}} f\left(\theta_{A}, \theta_{B} ; \rho\right) d \theta_{B} d \theta_{A} \\
= & {\left[\int_{p_{B}}^{+\infty} \int_{\theta_{B}-p_{B}+p_{A}}^{+\infty} f\left(\theta_{A}, \theta_{B} ; \rho\right) d \theta_{A} d \theta_{B}+\int_{p_{A}}^{+\infty} \int_{-\infty}^{p_{B}} f\left(\theta_{A}, \theta_{B} ; \rho\right) d \theta_{B} d \theta_{A}\right]\left(p_{A}-c\right) }
\end{aligned}
$$

By taking the first order derivative, we find that the price in a symmetric equilibrium must satisfy:

$$
p^{*}=\frac{\int_{p^{*}}^{+\infty} \int_{\theta_{B}}^{+\infty} f\left(\theta_{A}, \theta_{B} ; \rho\right) d \theta_{A} d \theta_{B}+\int_{p^{*}}^{+\infty} \int_{-\infty}^{p^{*}} f\left(\theta_{A}, \theta_{B} ; \rho\right) d \theta_{A} d \theta_{B}}{\int_{p^{*}}^{+\infty} f\left(\theta_{B}, \theta_{B} ; \rho\right) d \theta_{B}+\int_{-\infty}^{p^{*}} f\left(p^{*}, \theta_{B} ; \rho\right) d \theta_{B}}+c .
$$

Denote the firm's single-period profit in the symmetric equilibrium by $\pi^{*}$. Note that in the sub-game perfect equilibrium of the two-period normal goods pricing game, firms play the single-period Nash equilibrium in each period. Thus, each firm makes a profit equals $(1+\delta) \pi^{*}$ in the two-stage pricing game. So, for sufficiently small $\delta$, firms in the current setting make a greater profit than in the main model, which is given by $\delta \pi^{S *}$. The intuition is as follows: In the main model, firms compete in the first period for differential profits in the second period. When firms are sufficiently impatient, they are less concerned about future differential profits, and focus on the first period profit, which is, hence, competed down to close to zero. In the normal goods benchmark, firms are already differentiated in the first period, which allows them to earn a profit in that period.

\section{F Asymmetric distribution}

While the assumption of a symmetric distribution of WTP in our model is common in the literature, we discuss the implications of an asymmetric value distribution in this section. Rather than providing a detailed characterization of second-period equilibria, we emphasize the first period equilibrium and firm profits.

We focus on the case where the marginal distributions of WTPs for both brands have either a sufficiently large mean, such that both firms' second-period markets have the mass market equilibrium, or a sufficiently small mean, such that both have the niche market equilibrium. Consider the former case. Denote firm $i$ 's second-period profit from repeat consumers by $\pi_{i}^{R}$ and the profit from switching consumers by $\pi_{i}^{S}$. Suppose $\pi_{A}^{R}-\pi_{A}^{S} \geq \pi_{B}^{R}-\pi_{B}^{S}>0$ and consumers' second-period surpluses are the same across products. Then, firms compete for greater market share in the first period, and thus, the first period price must be $c-\delta\left(\pi_{B}^{R}-\pi_{B}^{S}\right)$. This is the 
case because firm 2 would stop reducing price at this point to avoid an overall loss in the game. In such an equilibrium, firm 2 makes zero profit while firm 1 makes a positive profit of

$$
\lambda_{A}\left[c-\delta\left(\pi_{B}^{R}-\pi_{B}^{S}\right)-c\right]+\delta\left(\lambda_{A} \pi_{A}^{R}+\lambda_{B} \pi_{A}^{S}\right)=\delta \lambda_{A}\left(\pi_{A}^{R}-\pi_{B}^{R}\right)+\delta\left(\lambda_{A} \pi_{B}^{S}+\lambda_{B} \pi_{A}^{S}\right) .
$$

Note that when the marginal distributions are similar in that the difference between the means and between the variances are sufficiently small, the term $\left(\pi_{A}^{R}-\pi_{B}^{R}\right)$ is sufficiently close to zero, whereas the term $\left(\lambda_{A} \pi_{B}^{S}+\lambda_{B} \pi_{A}^{S}\right)$ is sufficiently close to $\pi_{A}^{S}$, consistent with Theorem 1 .

The case when both firms have the niche market equilibrium in the second period is similar. Recall that firms make the monopoly profit in the niche market equilibrium. Denote firm $i$ 's monopoly profit by $\pi_{i}^{M}$. ${ }^{30}$ Suppose $\pi_{A}^{M} \geq \pi_{B}^{M}$. Then, following a similar logic, the first period price is given by $c-\delta \pi_{B}^{M}$. Firm 2 makes zero profit, whereas firm 1's profit is

$$
\lambda_{A}\left(c-\delta \pi_{B}^{M}-c\right)+\delta \lambda_{A} \pi_{A}^{M}=\delta \lambda_{A}\left(\pi_{A}^{M}-\pi_{B}^{M}\right) .
$$

When the difference in the marginal distributions are sufficiently small, this profit is sufficiently close to zero, consistent with Theorem 1.

\section{G Second-period equilibria under uniform pricing}

Now we turn to uniform pricing, where firms cannot price discriminate consumers based on their purchasing history. Denote by $U_{i}^{k}$ with $k \in\{m, n\}$ the uniform price of firm $i$ in a mass $(k=m)$ or a niche $(k=n)$ market equilibrium. Note that under uniform pricing, we have $R_{i}=S_{i}=U_{i}$ in the profit function (5).

\section{G.1 Without information spillovers, i.e., $\rho=0$}

When $\rho=0$, we can derive the equilibrium second-period pricing using an approach similar to the approach taken in the main analysis. The equilibrium is characterized below.

Proposition 8. Suppose $\rho=0$ and behavior-based price discrimination is not allowed, in the second period there exists:

- a niche market equilibrium where $U_{i}^{n}=M$ if and only if $\mu<M$;

- a mass market equilibrium where $U_{i}^{m}=\frac{1}{2 f(\mu)}+c \geq M$ if and only if $\mu \geq M$.

\footnotetext{
${ }^{30} \pi_{i}^{M}$ is equivalent to $\pi(M)$ when the distributions of WTP is symmetric.
} 
An interesting observation we can make from Proposition 8 is that the semi-niche market equilibrium no longer exists. This suggests that it is behavior-based price discrimination that enables the existence of the semi-niche market equilibrium.

\section{G.2 With information spillovers, i.e., $\rho>0$}

Proposition 9 illustrates that the second period equilibrium under uniform pricing is also different from when behavior-based price discrimination is allowed, given that $\rho>0$. As in Proposition 8, there no longer exists any semi-niche market equilibrium.

Proposition 9. Suppose $\rho \in(0,1)$. In the second period, there exists:

- a niche market equilibrium where firm $i$ charges $U_{i}^{n}=M$ if and only if $\mu \leq M$;

- a mass market equilibrium where firm $i$ charges

$$
U_{i}^{m}=\frac{\rho(1-\rho)\left[1-F\left(\theta_{-i}^{S O}\right)\right]}{2 \rho f(\mu)+(1-\rho) f\left(\theta_{-i}^{S O}\right)}+c, \quad \text { where } \theta_{-i}^{S O}=\frac{U_{i}^{m}-(1-\rho) \mu}{\rho}
$$

if and only if

$$
\mu-\frac{1-\rho}{2 f(\mu)} \frac{\rho}{1+\rho}-c \geq 0
$$

Note that $\mu>M$ implies that (58) holds, suggesting that the mass market equilibrium exists whenever the niche market equilibrium does not exist. Correspondingly, violation of (58) implies that $\mu \leq M$, suggesting the existence of the niche market equilibrium whenever the mass market equilibrium does not exist.

Proposition 9 indicates that when $\mu$ satisfies (58) and $\mu \leq M$, both the mass and the niche market equilibrium exist. Again, semi-niche market equilibrium no longer exists when price discrimination is not allowed.

\section{G.3 Proofs of second-period equilibria under uniform pricing}

Proof of Proposition 8. We consider each equilibrium in turn:

Step 1: Niche market equilibrium

In the niche market equilibrium, there are no switching customers. Each firm finds it optimal to charge the monopoly price $M$ to its repeat customers.

When the type $\theta_{i}=M$ switches to $-i$, she has a surplus of $\mu-M$. Then, a sufficient condition for the existence of the niche market equilibrium is that $\mu<M$, as type $\theta_{i}=M$ and 
all types with higher valuations must find it unprofitable to switch. Suppose firm $i$ deviates to a lower price $U$. If $U \geq \mu$ holds, then no consumers switch. If instead $U<\mu$, then firm $i$ 's profit function now becomes

$$
(U-c)\left[\lambda_{-i} \int_{-\infty}^{\mu-U+M} d F\left(\theta_{-i}\right)+\lambda_{i} \int_{U}^{+\infty} d F\left(\theta_{i}\right)\right]
$$

First order derivative w.r.t $U$ yields

$$
\begin{aligned}
& \lambda_{-i} F(\mu-U+M)\left[1-\frac{(U-c) f(\mu-U+M)}{F(\mu-U+M)}\right]+\lambda_{i}[1-F(U)]\left[1-\frac{(U-c) f(U)}{1-F(U)}\right] \\
> & \left\{\lambda_{-i} F(\mu-U+M)+\lambda_{i}[1-F(U)]\right\}[1-2(U-c) f(\mu)] \\
> & 0
\end{aligned}
$$

which the first inequality is due to $U<\mu$ and $U<M$, and the second inequality is due to $U<\mu<\frac{1}{2 f(\mu)}+c$.

Now we turn to necessity. Suppose the niche market equilibrium exists, then switching must yield negative surplus, i.e., $\mu-M<0$.

Step 2: Mass market equilibrium

For the mass market equilibrium, firm $i$ 's profit function from charging $U_{i}$ is given by:

$$
\begin{aligned}
& \left(U_{i}-c\right) \cdot\left[\lambda_{i} \int_{\theta_{i}^{R S}}^{+\infty} d F\left(\theta_{i}\right)+\lambda_{-i} \int_{-\infty}^{\theta_{-i}^{R S}} d F\left(\theta_{-i}\right)\right] \\
= & \left(U_{i}-c\right) \cdot\left\{\lambda_{i}\left[1-F\left(\mu+\frac{U_{i}-U_{-i}}{1-\rho}\right)\right]+\lambda_{-i} F\left(\mu+\frac{U_{-i}-U_{i}}{1-\rho}\right)\right\} .
\end{aligned}
$$

Take the first order derivative w.r.t $U_{i}$, we have

$$
\begin{aligned}
& \lambda_{i} \cdot\left[1-F\left(\mu+\frac{U_{i}-U_{-i}}{1-\rho}\right)\right]+\lambda_{-i} \cdot F\left(\mu+\frac{U_{-i}-U_{i}}{1-\rho}\right) \\
& -\frac{U_{i}-c}{1-\rho}\left[\lambda_{i} \cdot f\left(\mu+\frac{U_{i}-U_{-i}}{1-\rho}\right)+\lambda_{-i} \cdot f\left(\mu+\frac{U_{-i}-U_{i}}{1-\rho}\right)\right]=0
\end{aligned}
$$

We look for symmetric equilibrium where $U_{i}=U_{-i}$, then the first order condition (59) implies that $U_{i}^{m}=\frac{1}{2 f(\mu)}+c$.

Since the marginal consumer has a WTP of $\mu$, the mass market equilibrium exists if and only if $\mu-U_{i}^{m} \geq 0$, i.e.,

$$
\mu-\frac{1}{2 f(\mu)}-c \geq 0
$$

which is equivalent of $\mu \geq M$. 
Proof of Proposition 9. In a niche market equilibrium, it must be true that the marginal consumer leaves the market, i.e., $\theta_{i}^{R S}-U_{i}^{n}<0$. Since no consumer switches in the niche market equilibrium, each firm must charge the monopoly price $M$. No consumer wishes to switch, if and only if the surplus of the consumer with $\theta_{i}=M$ from switching is non-positive: $\rho M+(1-\rho) \mu-M \leq 0$, i.e., $\mu \leq M$.

In a mass market equilibrium, it must hold that the marginal consumer switches instead of leaving the market. To prove sufficiency, we show that when the condition given in the proposition holds, it must be true that $\theta_{i}^{R S}-U_{i}^{m} \geq 0$. First, note that the equilibrium is symmetric, hence $\theta_{i}^{R S}=\mu$. Thus,

$$
\begin{aligned}
\theta_{i}^{R S}-U_{i}^{m} & =\mu-U_{i}^{m} \\
& =\mu-\frac{\rho(1-\rho)\left[1-F\left(\theta_{-i}^{S O}\right)\right]}{2 \rho f(\mu)+(1-\rho) f\left(\theta_{-i}^{S O}\right)}-c \\
& \geq \mu-\frac{\rho(1-\rho)}{1+\rho} \frac{1-F\left(\theta_{-i}^{S O}\right)}{f\left(\theta_{-i}^{S O}\right)}-c,
\end{aligned}
$$

where the inequality is due to $f\left(\theta_{-i}^{S O}\right) \leq f(\mu)$. If $\theta_{-i}^{S O}>\mu$, then $\frac{1-F\left(\theta_{-i}^{S O}\right)}{f\left(\theta_{-i}^{S O}\right)} \leq \frac{1}{2 f(\mu)}$, which then implies that (60) satisfies

$$
\mu-\frac{\rho(1-\rho)}{1+\rho} \frac{1-F\left(\theta_{-i}^{S O}\right)}{f\left(\theta_{-i}^{S O}\right)}-c \geq \mu-\frac{1-\rho}{2 f(\mu)} \frac{\rho}{1+\rho}-c \geq 0
$$

This implies that $\mu \geq U_{i}^{m}$. However, given that $\theta_{-i}^{S O}>\mu$, we have $U_{i}^{m} \equiv \rho \theta_{-i}^{S O}+(1-\rho) \mu>\mu$. A contradiction. Hence, $\mu \geq \theta_{-i}^{S O}$ must hold, which implies that $U_{i}^{m} \equiv \rho \theta_{-i}^{S O}+(1-\rho) \mu \leq \mu$.

Now we turn to necessity. Given that $\mu-U_{i}^{m} \geq 0$,

$$
\begin{aligned}
\mu-\frac{1-\rho}{2 f(\mu)} \frac{\rho}{1+\rho}-c \geq \mu-\frac{\frac{1}{2} \rho(1-\rho)}{2 f(\mu)} \frac{\rho}{1-\rho}-c & \geq \mu-\frac{\rho(1-\rho)\left[1-F\left(\theta_{-i}^{S O}\right)\right]}{2 \rho f(\mu)+(1-\rho) f\left(\theta_{-i}^{S O}\right)}-c \\
& \geq \mu-U_{i}^{m} \geq 0
\end{aligned}
$$

holds. Hence, we have proved the second part of the proposition.

Note that whenever $\mu>M$ holds such that the niche market does not exist, we have

$$
\begin{aligned}
\mu-\frac{1}{2 f(\mu)}-c>0 & \Rightarrow \mu-\frac{1}{2 f(\mu)} \frac{\rho}{1+\rho}-c>0 \\
& \Rightarrow \mu-\frac{1}{2 f(\mu)} \frac{\rho}{1+\rho}+\frac{\rho^{2}}{1+\rho}-c>0,
\end{aligned}
$$

which implies (58) holds, i.e., a mass market equilibrium exists. Alternatively, when (58) is violated, $\mu<M$ must be true. 


\section{References}

Beggs, A. and P. Klemperer (1992). Multi-period competition with switching costs. Econometrica: Journal of the Econometric Society, 651-666.

Bergemann, D. and J. Välimäki (1997). Market diffusion with two-sided learning. The RAND Journal of Economics, 773-795.

Bergemann, D. and J. Välimäki (2006). Dynamic pricing of new experience goods. Journal of Political Economy 114(4), 713-743.

Bester, H. (1998). Quality uncertainty mitigates product differentiation. The RAND Journal of Economics, 828-844.

Bloom, N., M. Schankerman, and J. Van Reenen (2013). Identifying technology spillovers and

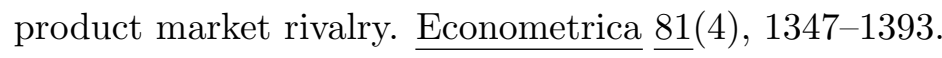

Chen, Y. (1997). Paying customers to switch. Journal of Economics \& Management Strategy $\underline{6}(4), 877-897$.

Chen, Y. and J. Pearcy (2010). Dynamic pricing: when to entice brand switching and when to reward consumer loyalty. The RAND Journal of Economics 41(4), 674-685.

Cremer, J. (1984). On the economics of repeat buying. The RAND Journal of Economics, 396-403.

Doganoglu, T. (2010). Switching costs, experience goods and dynamic price competition. QME $\underline{8}(2), 167-205$.

Farrell, J. and P. Klemperer (2007). Coordination and lock-in: Competition with switching costs and network effects. Handbook of industrial organization 3, 1967-2072.

Farrell, J. and C. Shapiro (1988). Dynamic competition with switching costs. The RAND Journal of Economics, 123-137.

Fudenberg, D. and J. Tirole (2000). Customer poaching and brand switching. RAND Journal of Economics, 634-657.

Gehrig, T. and R. Stenbacka (2004). Differentiation-induced switching costs and poaching. Journal of Economics \& Management Strategy 13(4), 635-655. 
Hannak, A., G. Soeller, D. Lazer, A. Mislove, and C. Wilson (2014). Measuring price discrimination and steering on e-commerce web sites. In Proceedings of the 2014 conference on internet measurement conference, pp. 305-318.

Ivanov, M. (2009). Niche market or mass market? Economics letters 105(3), 217-220.

Jing, B. (2011). Pricing experience goods: The effects of customer recognition and commitment. Journal of Economics \& Management Strategy 20(2), 451-473.

Klemperer, P. (1987a). The competitiveness of markets with switching costs. The RAND Journal of Economics, 138-150.

Klemperer, P. (1987b). Markets with consumer switching costs. The quarterly journal of economics $\underline{102}(2), 375-394$.

Klemperer, P. (1995). Competition when consumers have switching costs: An overview with applications to industrial organization, macroeconomics, and international trade. The review of economic studies $\underline{62}(4), 515-539$.

Moretti, E. (2011). Social learning and peer effects in consumption: Evidence from movie sales. The Review of Economic Studies 78

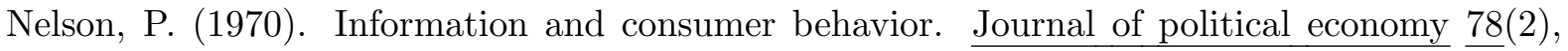
$311-329$.

Padilla, A. J. (1992). Mixed pricing in oligopoly with consumer switching costs. International Journal of Industrial Organization 10(3), 393-411.

Padilla, A. J. (1995). Revisiting dynamic duopoly with consumer switching costs. Journal of Economic Theory $67(2), 520-530$.

Villas-Boas, J. M. (2004). Consumer learning, brand loyalty, and competition. Marketing $\underline{\text { Science }} \underline{23}(1), 134-145$.

Villas-Boas, J. M. (2006). Dynamic competition with experience goods. Journal of Economics

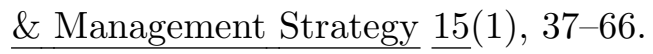

